\title{
Aortic intimal resident macrophages are essential for maintenance of the non-thrombogenic intravascular state
}

\author{
Gloria E. Hernandez ${ }^{1,2}$, Feiyang Ma ${ }^{1}$, Guadalupe Martinez ${ }^{3}$, Nadia B. Firozabadi², \\ Jocelynda Salvador ${ }^{2}$, Lih Jiin Juang ${ }^{4}$, Jerry Leung ${ }^{4}{ }^{4}$, Peng Zhao $\mathbb{1}^{5}$, Diego A. López ${ }^{6}{ }^{6}$, \\ Reza Ardehali ${ }^{5}{ }^{5}$, Anna E. Beaudin7, Christian J. Kastrup ${ }^{4}$, Matteo Pellegrini ${ }^{1,3}$, Matthew J. Flick ${ }^{8}$ \\ and M. Luisa Iruela-Arispe $\mathbb{B}^{2,3 凶}$
}

Leukocytes and endothelial cells frequently cooperate to resolve inflammatory events. In most cases, these interactions are transient in nature and triggered by immunological insults. Here, we report that, in areas of disturbed blood flow, aortic endothelial cells permanently and intimately associate with a population of specialized macrophages. These macrophages are recruited at birth from the closing ductus arteriosus and share the luminal surface with the endothelium, becoming interwoven in the tunica intima. Anatomical changes that affect hemodynamics, such as in patent ductus arteriosus, alter macrophage seeding to coincide with regions of disturbed flow. Aortic resident macrophages expand in situ via direct cell renewal. Induced depletion of intimal macrophages leads to thrombin-mediated endothelial cell contraction, progressive fibrin accumulation and formation of microthrombi that, once dislodged, cause blockade of vessels in several organs. Together the findings reveal that intravascular resident macrophages are essential to regulate thrombin activity and clear fibrin deposits in regions of disturbed blood flow.

A $s$ a gatekeeper of cellular traffic between blood and tissues, the endothelium is well equipped to interact with hematopoietic cells. Endothelial cells enable the well-coordinated process of diapedesis that includes capture, rolling and finally transmigration of leukocytes across the vascular barrier ${ }^{1-4}$. These events rely on complex and sequential molecular interactions by which these cell types cooperate to mount and resolve inflammatory responses at the level of venules and capillaries ${ }^{2,3}$. In large arteries, interactions of the endothelium with inflammatory cells are mostly known for their association with atherosclerosis, a chronic inflammation of the vascular wall ${ }^{5,6}$. In this pathology, leukocytes occupy the subendothelial layer, forming a neointima that progressively expands by the constant influx and local proliferation of inflammatory cells ${ }^{5,7-9}$.

Other types of interactions between arterial endothelium and inflammatory cells have been shown, particularly a population of non-classical monocytes (Ly6 $\mathrm{C}^{\mathrm{lo}} \mathrm{CCR} 2{ }^{\mathrm{lo}} \mathrm{CX} 3 \mathrm{CR} 1^{\text {hi }}$ ) better referred to as patrolling monocytes that are thought to promote endothelial integrity and vascular health ${ }^{10-12}$. In addition to patrolling monocytes, myeloid cells with a highly dendritic appearance were also described in the luminal aspect of the aorta, especially at sites prone to develop atherosclerosis, such as the aortic $\operatorname{arch}^{13,14}$. Despite their conspicuous location, the contribution of these myeloid cells to atherosclerosis was proved to be minimal, as their elimination only slightly delayed onset of the disease with no impact on duration or burden ${ }^{15}$. Thus, the mechanisms behind their peculiar distribution, specific seeding time and, more importantly, their function have remained puzzling.

Here we show that the emergence of aortic myeloid cells is not pathologically induced; instead, it is developmentally triggered as part of natural hemodynamic changes at birth that result in localized disturbed flow dynamics. Genetic ablation of this aortic myeloid resident population promotes fibrin deposition and microthrombus formation, clarifying its function as a critical regulator of hemostasis.

\section{Results}

Hemodynamics at birth promote seeding of myeloid cells in the tunica intima of the aorta. Fetal circulation includes two parallel circuits with equal left and right ventricular pressures. At birth, this balance changes drastically due to multiple concurrent events that include interruption of placental circulation, inflation of the lungs and a shift in pulmonary blood pressure. These changes result in high left ventricular pressure and closure of the ductus arteriosus, a fetal vessel that connects the pulmonary arteries to the aorta, an event that further magnifies oscillatory flow in the lower curvature of the aortic arch (Fig. 1a). These alterations in hemodynamics are quickly sensed by endothelial cells, which transition from an elongated to a polygonal shape in the lesser curvature of the aortic

${ }^{1}$ Molecular Biology Institute, University of California, Los Angeles, Los Angeles, CA, USA. ²Department of Cell and Development Biology, Feinberg School of Medicine, Northwestern University, Chicago, IL, USA. ${ }^{3}$ Department of Molecular, Cell and Developmental Biology, University of California, Los Angeles, Los Angeles, CA, USA. ${ }^{4}$ Michael Smith Laboratories and Department of Biochemistry and Molecular Biology, University of British Columbia, Vancouver, British Columbia, Canada. ${ }^{5}$ Department of Medicine, Division of Cardiology, University of California, Los Angeles, Los Angeles, CA, USA. ${ }^{6}$ Department of Pathology, University of Utah School of Medicine, Salt Lake City, UT, USA. 'Division of Hematology and Hematologic Malignancies, Molecular Medicine Program, Department of Internal Medicine, University of Utah School of Medicine, Salt Lake City, UT, USA. ${ }^{8}$ Department of Pathology and Laboratory Medicine, UNC Blood Research Center, University of North Carolina, Chapel Hill, Chapel Hill, NC, USA.凶e-mail: arispe@northwestern.edu 
arch (Fig. 1b,c). Furthermore, evaluation of mouse embryos and neonates uncovered a burst of inflammatory cells exiting from the constricted ductus arteriosus that seeds the aorta, in tandem with the changes in hemodynamics (Fig. 1d-f). Interestingly, from the onset of birth, this population of $\mathrm{CD} 45^{+}$cells continued to reside in areas experiencing oscillatory and disturbed flow, including the lesser curvature of the aortic arch and branch openings (Fig.1g-i and Extended Data Fig. 1a). Initial characterization indicated that they also expressed CD11c (Fig. 1h,j). Curiously, CD11 $\mathrm{c}^{+}$cells were previously detected in the aortic arch of adult healthy mice ${ }^{13,14}$, raising the possibility that they might be the same population. We also found that $\mathrm{CD} 11 \mathrm{c}^{+}$cells progressively accumulated with age in the absence of pathologies or hypercholesterolemia (Extended Data Fig. 1a-c). However, they were not found in large veins, such as the vena cava (Extended Data Fig. 1d,e), indicating that an arterial niche, including flow patterns, may be required for their accumulation. Intimal immune cells were also absent from the carotid arteries of healthy, adult mice (Extended Data Fig. 1f) and from the descending young aorta, except for branches (Extended Data Fig. 1g). We also observed that the distribution of these cells progressively broadened with age. In fact, the descending aorta of 52and 78-week-old mice revealed ongoing accumulation of intimal immune cells even in areas of laminar flow (Extended Data Fig. 1g-i), suggesting that vascular aging might also be a supportive niche for the seeding of intimal myeloid cells.

A definitive link between onset of oscillatory flow and recruitment of CD11 $\mathrm{c}^{+}$cells was established using mouse models of patent ductus arteriosus (PDA). Failure in PDA closure significantly alters cardiovascular hemodynamics ${ }^{16}$. While viable and fertile ${ }^{17}$, Vimentin ${ }^{-1-}\left(\mathrm{Vim}^{-1-}\right)$ mice exhibit PDA in about $88 \%$ of adults, making them an ideal model to study myeloid cell distribution in adult aortae ${ }^{18}$. At 10 weeks of age, the ductus arteriosus in wild-type (WT) littermates becomes a solid fibrous structure that persists as the ligamentum arteriosum (Fig. 1k). By contrast, $\mathrm{Vim}^{-/-}$mice exhibit a viable ductus arteriosus, which impacts patterns of disturbed flow and the location of myeloid cells (Fig. 11). The lesser curvature of the aortic arch in $\mathrm{Vim}^{-/-}$mice showed no intimal myeloid accumulation; instead, myeloid cells were noted, surrounding the openings of the ductus arteriosus, the subclavian artery and onset of the descending aorta (Fig. 11).

The thin nature of the endothelial lining makes it difficult to ascertain the precise topology of myeloid cells in relation to the endothelium. Using $C d h 5^{\text {CreERT2}} ; R 26^{\text {tdTomato }}$ reporter mice to label the endothelial monolayer (tdTomato combined with CD45 and vascular endothelial cadherin (VE-Cad) staining), we generated a three-dimensional (3D) rendering of the tunica intima. Myeloid cells were neither above nor below the endothelium but were instead interwoven within the endothelial monolayer, with cell processes projecting into and others below the lumen (Fig. 2a). En face scanning electron microscopy (SEM) of the aortic arch stained with anti-CD45 antibodies confirmed these findings (Fig. 2b). Additionally, 3D surface rendering using a reporter model that labels the tunica media using the Tagln (Sm22) promoter $\left(\mathrm{Sm} 22^{\mathrm{Cre}} ; \mathrm{R}^{26^{\text {tdTomato }}}\right)$ combined with VE-Cad immunohistochemistry further validated that these $\mathrm{CD} 45^{+}$cells were located in the tunica intima (Fig. 2c). Finally, myeloid cells were detected by intravascular injection of anti-CD45 antibodies in vivo (Fig. 2d,e).

During diapedesis, leukocytes adhere to endothelial cells through platelet endothelial cell adhesion molecule (PECAM1), preserving junctional integrity as they cross the endothelial barrier ${ }^{2-4}$. We also detected PECAM1 expression in these myeloid cells, indicating that they bind to the endothelium via homophylic, heterotypic interactions and prevent barrier disruption (Fig. 2f). En face images after injection of non-blocking PECAM1-specific antibodies revealed lumen-exposed regions in inflammatory cells (Fig. $2 \mathrm{~g}$ ). When combined, these experiments revealed that, in regions of disturbed flow, the constituency of the endothelial layer is enriched by a population of myeloid cells that intimately coexists with the endothelium in the absence of pathology and without breach of permeability.

scRNA-seq reveals the transcriptional identity of aortic intimal immune cells. To recover cellular identities independently of defined labeling strategies, we turned to single-cell RNA sequencing (scRNA-seq) using specimens that were not sorted by flow cytometry $^{19,20}$ (Extended Data Fig. 2a). Three independent scRNA-seq libraries were generated, capitalizing on regions with abundant numbers of intimal immune cells: the aortic arch of 8 -week-old C57BL/6 mice ('young arch') and the descending aorta (thoracic and abdominal) of 78-week-old C57BL/6 mice ('aged descending $A, B$ ') (Extended Data Fig. 2b-e and Supplementary Table 1). Using dimensionality reduction by $t$-distributed stochastic neighbor embedding ( $t$-SNE) analysis, we identified ten distinct cell types and assigned cellular identities (Extended Data Fig. $2 \mathrm{f}$ and Supplementary Tables 2,3) based on canonical lineage markers (Extended Data Fig. 2g,h). Within the ten cell types, two distinct macrophage populations were identified based on Fcgr1 (CD64) and Adgre1 (F4/80) expression (Extended Data Fig. 2h). One of the macrophage clusters expressed Lyve1 (encoding lymphatic endothelium hyaluronan receptor 1 (LYVE1)), F13a1 and Mrc1 (CD206), well-known markers of adventitia macrophages ${ }^{15,21-24}$ (Extended Data Fig. 2i,j), suggesting that this macrophage population came from the adventitia. While our isolation method enriches for cells in the tunica intima, few cells from the other aortic layers (adventitia and media) were also captured ${ }^{19,20}$ (Extended Data Fig. 2f,g).

Fig. 1 | Hemodynamic changes at birth result in the recruitment of immune cells to the tunica intima. a, Embryonic day (E)18.5, connection between pulmonary arteries and aorta through the ductus arteriosus. Postnatal day (P)1, the ductus arteriosus constricts, contributing to disturbed flow. Eight weeks, independent aorta and pulmonary artery. Red, high oxygen levels; blue, low oxygen levels; purple, intermediate oxygen levels. b, Schema illustrating aorta dissection. GC, greater curvature; LC, lesser curvature; desc., descending aorta. confocal images of the lesser curvature and descending aorta from mice at E18.5, P7 and 8 weeks. Elongation factor in the lesser curvature is on the adjacent graph (E18.5, $n=3 ; \mathrm{P7}, n=4 ; 8$ weeks, $n=5$ mice), MannWhitney $t$-test, mean \pm s.d., two tailed, $P<0.0001$ (exact), ${ }^{\star \star \star \star} P \leq 0.0001$; scale bar, $10 \mu \mathrm{m}$ ). d, Bright-field images of ductus arteriosus (DA) remodeling (scale bars, $150 \mu \mathrm{m}, \mathrm{E} 18.5$ ( $n=8$ embryos); $200 \mu \mathrm{m}, \mathrm{P} 1$ ( $n=10$ mice); $500 \mu \mathrm{m}, \mathrm{P} 3(n=12$ mice); and $1 \mathrm{~mm}, 3$ weeks $(n=12 \mathrm{mice})$ ). e, Exit of immune cells (green) from the ductus arteriosus. VE-Cad or the transcription factor ERG are indicated in red. Scale bars, $300 \mu \mathrm{m}$ and $70 \mu \mathrm{m}$ (e(i-iv)); $n=12 \mathrm{mice}$ per time point. f, Lumen of the ductus arteriosus (dotted white lines) at P0; immune cells are shown in green. Scale bar, $50 \mu m ; n=5$ mice. $\mathbf{g}$, Illustration indicating regions of disturbed flow where intimal immune cells (green) accumulate. Branch openings (dashed boxes) are shown in $\mathbf{h}, \mathbf{i}$. $\mathbf{h}$, Large branch openings with accumulation of intimal myeloid cells (CD11c, red; CD45, green) (white arrows). ERG staining (white) identifies endothelial nuclei. BA, brachiocephalic trunk; LCCA, left common carotid artery; LSA, left subclavian artery. Scale bars, $200 \mu \mathrm{m}$ (left) and $20 \mu \mathrm{m}$ (right); $n=15$ mice. i, Time course (1 week, 8 weeks, 78 weeks) of intimal CD45+ cell (green) deposition in intercostal arteries (yellow arrows). VE-Cad, red; intimal CD45+ cells, green. Scale bar, $50 \mu \mathrm{m} ; n=12$ ( 1 week), $n=498$ ( 8 weeks), $n=6$ ( 78 weeks) mice. $\mathbf{j}$, In total, $99.4 \%$ of intimal CD45+ cells in the lesser curvature are also labeled as $\mathrm{CD}_{11 \mathrm{c}^{+}}\left(n=3\right.$ mice, mean \pm s.d.). k, Bright-field images of control and $\mathrm{Vim}^{-/-}$aortae. Insets show the remnant (control) and PDA (Vim ${ }^{\prime-}$ ) with blood ( $n=8$ mice per group; scale bar, $1,300 \mu \mathrm{m}$ ). I, Aortic arch of 10-week-old control and Vim ${ }^{-/-} \mathrm{mice}$ immune cells in control (green arrows) and Vim ${ }^{-/-}$ (dotted pink ovals) mice are shown. Right, high magnification of squares $(\mathbf{I}(\mathrm{i}, \mathrm{ii})$ ). Scale bars, $500 \mu \mathrm{m}$ and $40 \mu \mathrm{m}(\mathbf{I}(\mathrm{i}, \mathrm{ii})$ ); $n=3 \mathrm{mice}$ per group. 
Therefore, we predicted that one of the two macrophage populations identified was from the adventitia. To test this prediction, we performed single-cell sequencing of dissected aortic adventitia (Extended Data Fig. 3a-g) and compared the two distinct myeloid populations identified. For this, we selected CD14-positive cells from the purified adventitia and intimal-enriched libraries (Extended Data Fig. 3c-e). This approach definitively confirmed the identity of the macrophage clusters from the aorta libraries. The $\mathrm{Lyve}^{+} \mathrm{Fcgrl}^{+}$ group, also present in libraries from the adventitia, represented typical adventitial macrophages, while the second cluster with distinct expression of Mmp12 and Mmp13 was unique to intima-enriched aortic libraries and absent from the adventitia (Extended Data Fig. 3f,g). Furthermore, we compared the $M m p 12^{+} M m p 13^{+}$aortic macrophage population to macrophages from the Tabula Muris
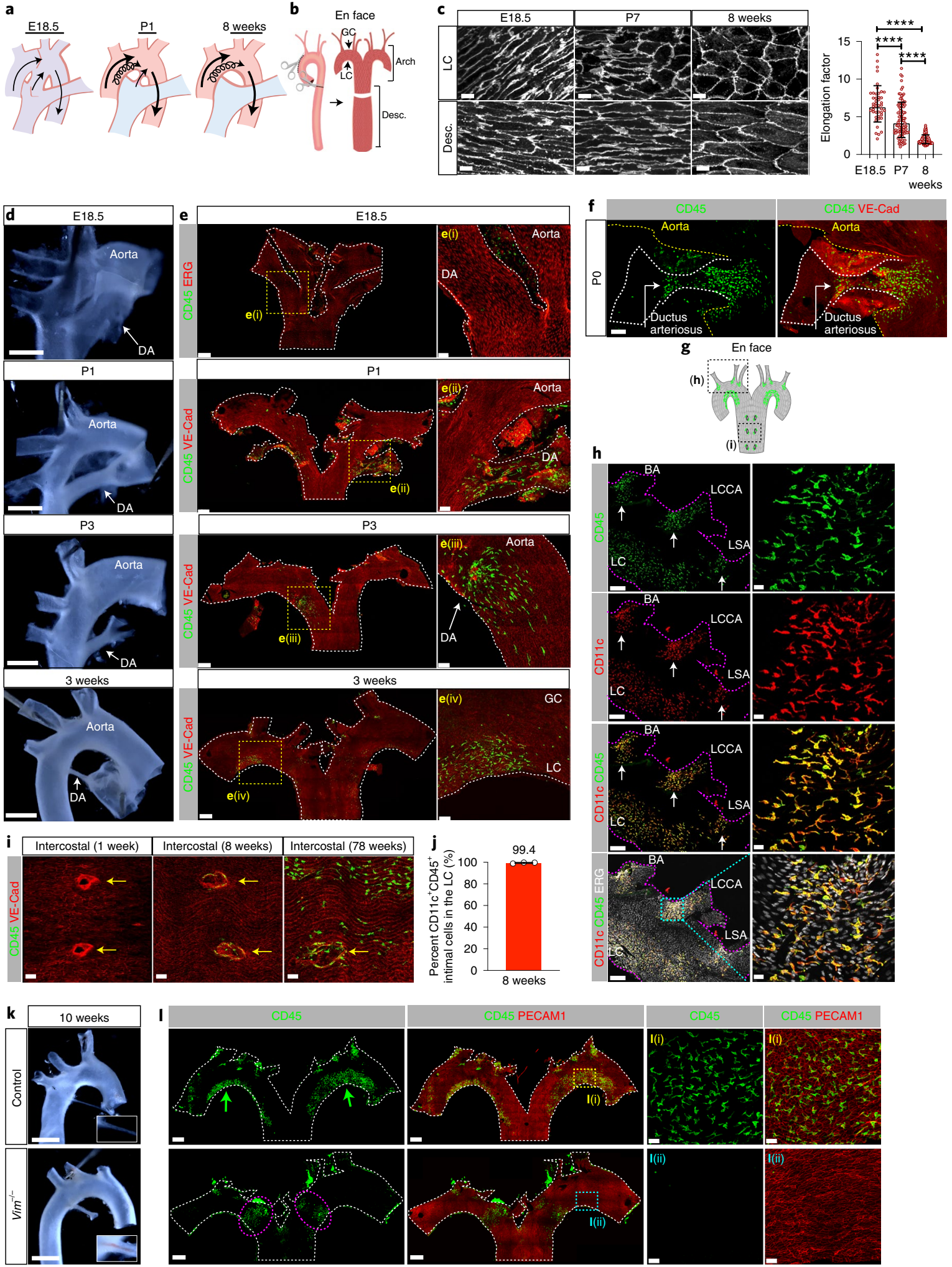
a

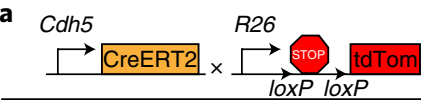
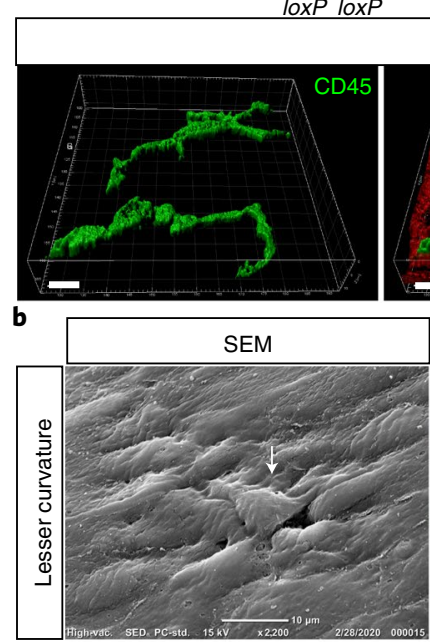

d

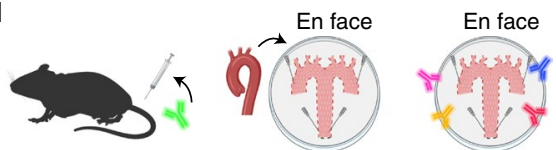

8 weeks $30 \mathrm{~min}$

i.v. inj. of rat anti-CD45

(e) or anti-PECAM1 (390)

(g) (label lumen-facing side)

f
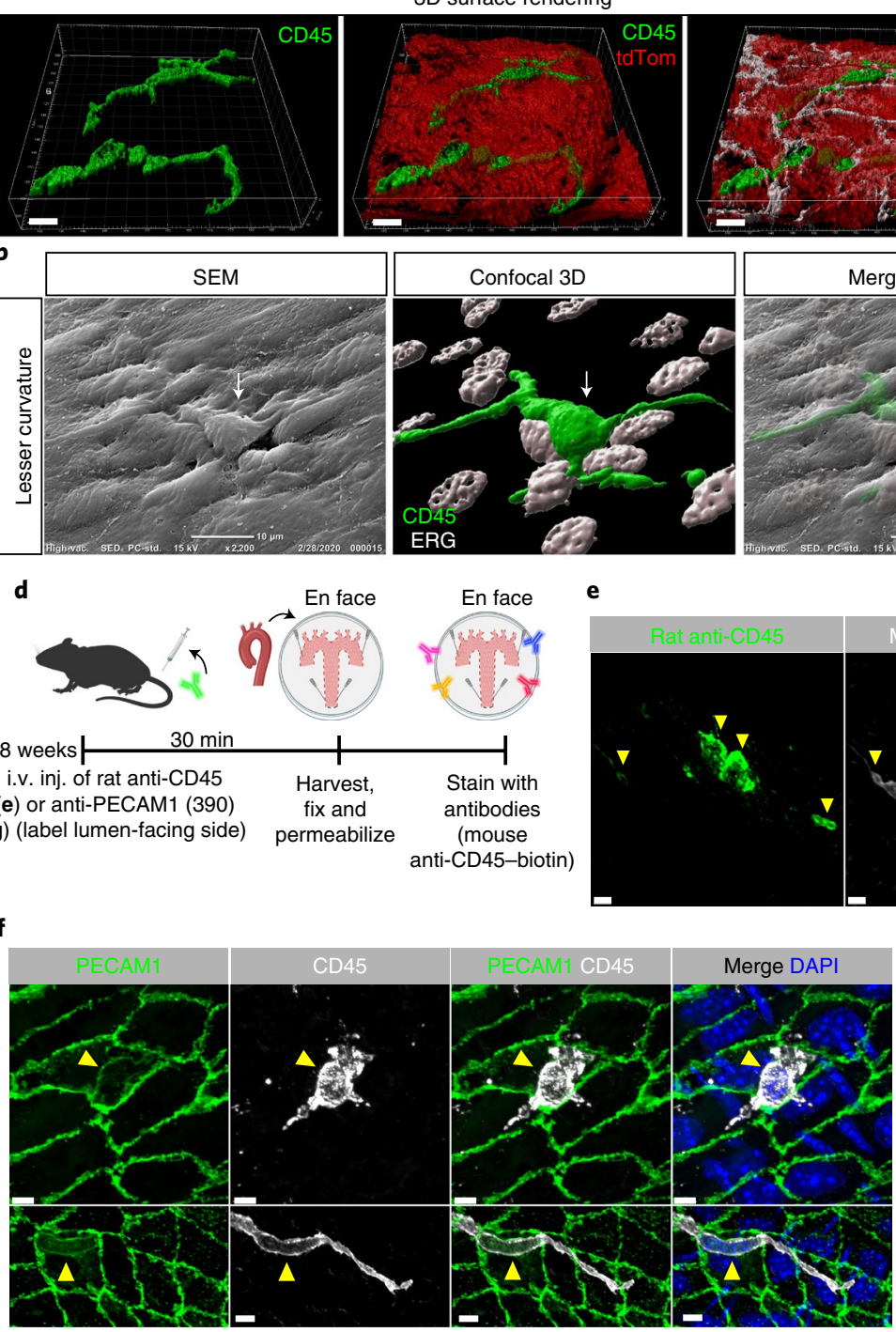

e c $_{\mathrm{Sm} 22} \quad \mathrm{R} 26$

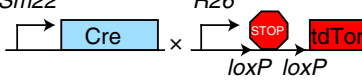

Max-int. proj.
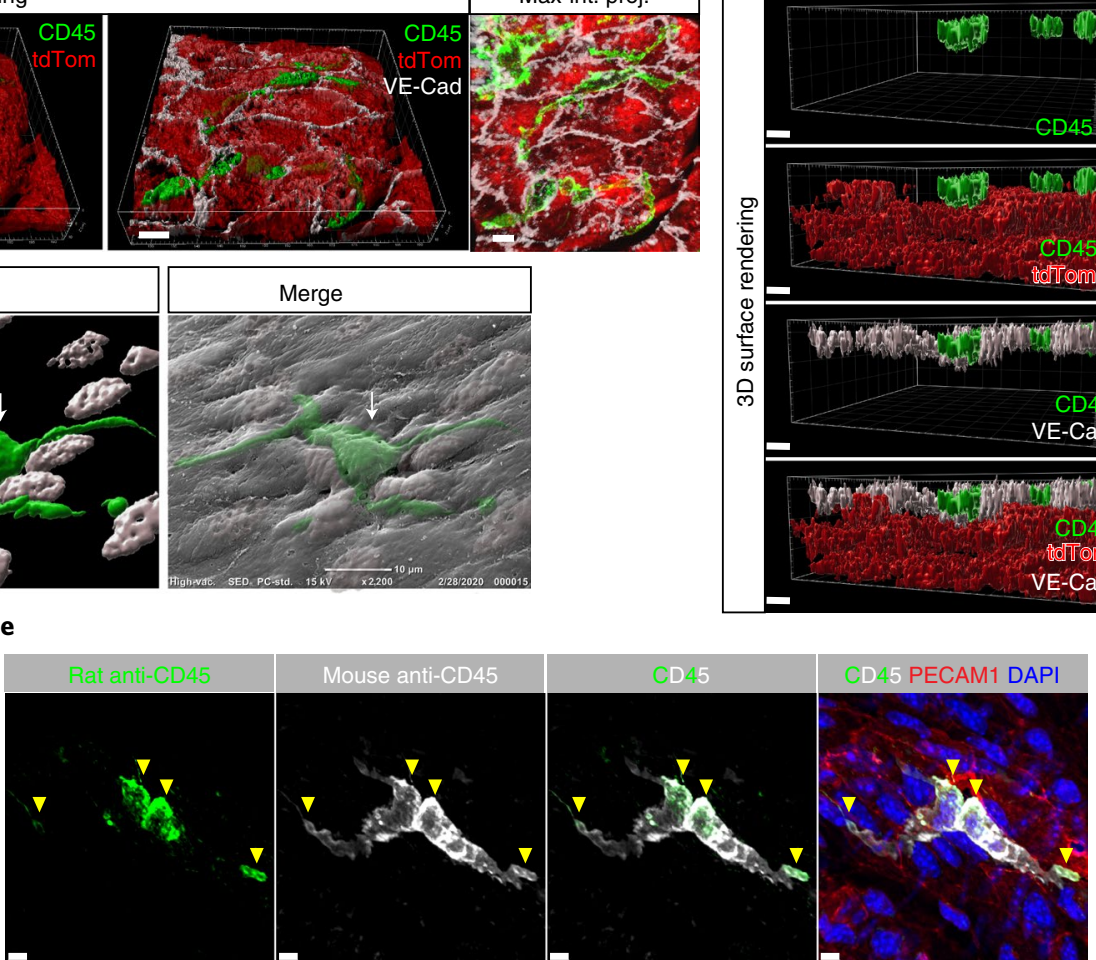

g
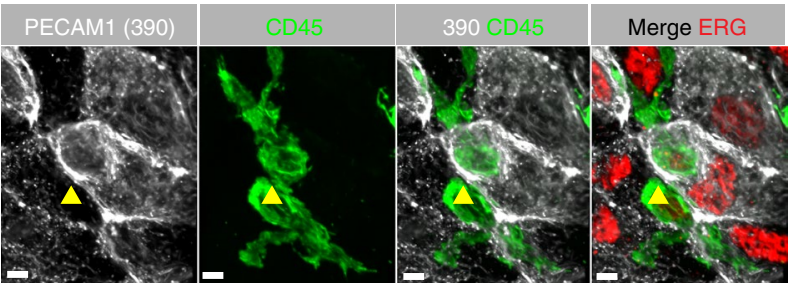

Fig. 2 | Topology of intimal myeloid cells in relation to the endothelium. $\mathbf{a}, C d h 5^{\mathrm{CreERT} 2} ; R 26^{\text {tdTomato }}$ construct of the double transgenic mice used for $3 \mathrm{D}$ surface rendering of adult aortae to visualize the spatial location of intimal CD $45^{+}$cells (green) relative to the endothelium (tdTomato (tdTom)). VE-Cad is shown in white. Scale bars, $8 \mu \mathrm{m} ; n=3$ mice. Max-int. proj., maximum-intensity projection. $\mathbf{b}$, Confocal and scanning electron microscopy (SEM) images of the same aorta were overlaid to determine the location of intimal CD45+ cells (green in the confocal view) in relation to the endothelium. ERG was used to visualize endothelial nuclei in confocal images. High-vac., high-vaccume. Scale bar, $10 \mu \mathrm{m} ; n=3$ mice. c, Sm22 $2^{\text {re }}$; $R 26^{\text {tdTomato }}$ construct of the double transgenic mice used for 3D rendering. Side view of a 3D surface image of adult aortae to visualize the spatial location of intimal CD45+ cells (green) relative to vascular smooth muscle cells (tdTom). Endothelial cells were visualized with anti-VE-Cad antibodies (white). Note that the green CD45+ cell is embedded in an area with white staining (VE-Cad). Scale bars, $5 \mu \mathrm{m} ; n=3$ mice. d, Schema of the experimental design related to e,g. Inj., injection; i.v., intravenous. e, Tail vein injection of rat anti-CD45 antibody (detected in green) was followed by euthanasia and fixation of the aorta 30 min later. Subsequent permeabilization and staining with an additional, distinct anti-CD 45 antibody (detected using far red, labeled in white). The illustration was created using https://biorender.com/.e, En face images of the experiment described. Yellow arrowheads indicate the portions of immune cells exposed to the lumen (green). Mouse anti-CD45 biotinylated antibody labeled with streptavidin-A647 used in immunostaining after permeabilization is shown in white. PECAM1, red; 4,6-diamidino-2-phenylindole (DAPI), blue. Scale bar, $5 \mu \mathrm{m} ; n=3$ mice. $\mathbf{f}$, En face images of the lesser curvature, staining for PECAM1 (green). Yellow arrowheads show faint PECAM1 positivity in CD45+ cells residing in the intima. Endothelial cells were detected in green with PECAM1. Scale bar, $5 \mu \mathrm{m} ; n=6$ mice. $\mathbf{g}$, C57BL/6 mice were injected intravenously with a non-blocking PECAM1-specific (390) antibody to further examine exposure of cell bodies to the lumen. Mice were killed and harvested 15-30 min post-injection. 15 min after injection. CD45 (in green) was used to identify immune cells, and ERG (red) was used to identify endothelial cells. Yellow arrowheads indicate PECAM1+ regions of intimal immune cells. Scale bar, $4 \mu \mathrm{m}$; $n=3$ mice.

Atlas $^{25}$ (Extended Data Fig. 4a-h) and resident macrophages characterized in more recent publications ${ }^{22}$ (Extended Data Fig. 4i). From this analysis, it became clear that the aortic population of intimal macrophages was especially distinct from resident macrophages in other organs, with the exception of sympathetic nerve-associated macrophages found in the lung ${ }^{22}$. 
Subsequently, we compared the $M m p 12^{+} M m p 13^{+}$macrophage population to a recently identified group of macrophages isolated from whole aortas and referred to as $\mathrm{Mac}^{\mathrm{AIR}}$ cells $^{15}$. Comparisons between the intimal $M m p 12^{+} M m p 13^{+}$population presented here to the pre-hypercholesterolemic aortic Mac ${ }^{\mathrm{AIR}}$ population ${ }^{15}$ revealed that they were transcriptionally identical (Supplementary Table 4). Interestingly, we also found that the young arch and old thoracic endothelial-associated macrophage populations were identical (Extended Data Fig. 4j and Supplementary Table 5), suggesting that the endothelial niche is responsible for the underlying tissue-specific imprinting of these macrophages. This realization prompted two immediate questions: were these populations progeny of $\mathrm{CD} 45^{+}$ cells exiting the ductus arteriosus? And, more importantly, what was their biological relevance?

Aortic intima resident macrophages $\left(\mathrm{Mac}^{\mathrm{AIR}}\right)$ are distinct macrophages that seed the aorta at birth. To clarify the function of $\mathrm{Mac}^{\mathrm{AIR}}$ cells and delve into their developmental origin, we first performed differential expression analysis to seek as many unique markers as possible. As shown by direct comparisons with adventitial macrophages and the Tabula Muris Atlas, we found that $\mathrm{Mac}^{\mathrm{AIR}}$ cells expressed significantly higher transcriptional levels of matrix metalloproteinases (MMPs) (Mmp12 and Mmp13; Extended Data Fig. 2i,j) and exhibited increased expression of immune cell activation genes (Illb, Ccl3, Ccl4, Tnf, Cxcl2 and Cxcl16; Extended Data Fig. 2i,j and Supplementary Tables 6-8). In addition, Mac ${ }^{\text {AIR }}$ cells displayed expression of genes involved with wound healing or identification of dying cells (Axl, Tyrobp, Cd44 and Cd74; Extended Data Fig. 2i,j and Supplementary Tables 6-8) and showed higher expression levels of antigen-presenting genes, such as major histocompatibility complex class II (MHC class II)-encoding genes (H2-Aa, H2-Ab1, H2-Eb1 and H2-M2; Extended Data Fig. 2i,j and Supplementary Tables 6-8). By contrast, adventitia Lyve $1^{\mathrm{hi}}$ macrophages expressed genes associated with M2-like macrophages (F13a1, Folr2 and Mrc1 (CD206); Extended Data Fig. 2i,j and Supplementary Tables 7 and 8).

To match the expression profile to their presumed progenitors, immunohistochemistry was performed on aortae of mice at postnatal day (P)7 and adult mice for targets unique to $\mathrm{Mac}^{\mathrm{AIR}}$ cells (MMP13, CXCL16 and CD11c). Intimal CD45 ${ }^{+}$cells colocalized with Mac ${ }^{\text {AIR }}$ markers (Extended Data Fig. 5a,b); whereas, in the adventitia, no $\mathrm{CD}_{4} 5^{+}$cells showed expression of $\mathrm{Mac}^{\mathrm{AIR}}$ markers (Extended Data Fig. 5c). We also found that Mac AIR cells expressed Cx3cr1 transcripts (Extended Data Fig. 5d) and CX3CR1 (fractalkine receptor) protein (Extended Data Fig. 5e).
Moreover, using an inducible $C x 3 c r 1$ reporter model $\left(C x 3 c r 1^{\text {CreERT2}}\right.$; $\left.R 26^{\text {tdTomato }}\right), 98 \%$ of all intimal $\mathrm{CD} 45^{+}$cells in adult aortae were labeled with tdTomato (Extended Data Fig. 5f), which also colocalized with CXCL16 (Extended Data Fig. 5g), thus demonstrating the activity of the $C \times 3 c r 1$ promoter, which was later used for lineage tracing. Additionally, using this transgene, intimal CD45 cells in aortae of mice at P5 were also labeled by the reporter after tamoxifen treatment (P1 and P3), further indicating that $\mathrm{Mac}^{\mathrm{AIR}}$ cells seed the aorta immediately after birth (Extended Data Fig. 5h). Overall, these findings support the conclusion that immune cells accumulating and residing in the tunica intima after birth were $\mathrm{Mac}^{\mathrm{AIR}}$ cells.

To more definitively confirm the origin and the macrophage identity of $\mathrm{Mac}^{\mathrm{AIR}}$ cells (versus dendritic cells (DCs)), we assessed recombination labeling using the $C s f 1 r^{\text {MerCreMer }} ; R 26^{\text {tTomato }}$ macrophage fate-mapping model. In this transgenic model, we found all intimal immune cells $\left(\mathrm{CD} 45^{+}\right)$to also be positive for tdTomato (Extended Data Fig. 5i). Moreover, all intimal immune cells expressed CD68 (Extended Data Fig. 5j). Additionally, Mac $^{\text {AIR }}$ cells did not express the DC master regulator transcription factor encoded by Zbtb46 (ref. ${ }^{26}$ ) nor classical DC markers Cd8a, Ccr7 (Extended Data Fig. 5k), Cd103, Dcir2, or Mycl (not detected). Furthermore, Mac ${ }^{\text {AIR }}$ cells were shown to phagocytose dying $\left(\right.$ Annexin ${ }^{+}$) endothelial cells in vivo (Extended Data Fig. 51), providing functional evidence of their macrophage identity. Thus, $\mathrm{Mac}^{\mathrm{AIR}}$ cells are a transcriptionally unique macrophage population that takes residency in the tunica intima of the aorta shortly after birth in regions of disturbed flow.

Mac $^{\mathrm{AIR}}$ cells are self-maintained throughout adulthood. It is now understood that many tissue-resident macrophages are maintained through self-renewal without contribution from circulating monocytes $^{27,28}$. Incorporation of 5-ethynyl-2-deoxyuridine (EdU) demonstrated that, in a 2 -h pulse, $5.4 \%$ of $\mathrm{Mac}^{\mathrm{AIR}}$ cells were undergoing DNA replication in situ in the adult arch (Extended Data Fig. 6a-c), suggesting that $\mathrm{Mac}^{\mathrm{AIR}}$ cells followed the trend of other resident macrophages. Long-term replenishment was assessed by lineage tracing with pulse-labeling $C \times 3 c r 1^{\text {CreERT2 }} ; R 26^{\text {thTomato }}$ mice and evaluation 10 and 20 weeks after labeling (Extended Data Fig. 6d,e). To control for the possible contribution of circulating cells, we also quantified tdTomato-positive blood cells. Flow cytometry analyses indicated that, after 3 weeks, CD $45^{+}$tdTomato $^{+}$peripheral blood was negligible (less than 0.5\%; Extended Data Fig. 6f,g), thus negating the possibility that cells from the circulation contributed to reporter positive Mac ${ }^{\text {AIR }}$ cells 20 weeks after tamoxifen treatment. In this manner, the absence of circulating tdTomato ${ }^{+}$cells allowed us to ask

Fig. 3 | Aortic intimal macrophages seed the aorta immediately after birth and self-renew in areas of oscillatory and disturbed flow. a, Experimental design. EdU injection at P7 $2 \mathrm{~h}$ before euthanasia. Intimal CD45+ cells (green) proliferate (EdU ${ }^{+}$, white) in situ (yellow arrowheads). Endothelial cells are shown in red (ERG). Scale bar, $20 \mu \mathrm{m} ; n=3$ mice. b, For lineage tracing, $C \times 3 c$ TreERT2; $^{\text {C } 26^{\text {tdTomato }}}$ neonates were injected with tamoxifen (tam) at P1, P3 and P5. At P7, aortae were collected from half of the litter as a baseline control. At 4 weeks, aortae were collected from the remaining littermates for retention of the reporter in Mac $\mathrm{MIR}^{\mathrm{A}}$ cells. c, Illustration depicting possible outcomes. tdTomato levels are negligible in blood (as shown in $\mathbf{g}$ ). PB, peripheral blood. d, tdTomato ${ }^{+}$immune cells in aortae at P7 (top) and 4 weeks (bottom) of Cx3cr TreERT2;R26 ${ }^{\text {tdTomato }}$ mice after tamoxifen administration. Mac ${ }^{\text {AlR }}$ cells in aortae of $C \times 3 c r 7^{\text {CreERT2 }}, R 26^{\text {tdTomato }}$ mice at 4 weeks retained reporter (tdTomato ${ }^{+}$) expression when labeled postnatally, as shown by the comparison between $\mathrm{P} 7$ (baseline) and 4 weeks (lineage traced). CD45, green; VE-Cad, white. Scale bars, $80 \mu \mathrm{m}$ and $10 \mu \mathrm{m}, n=5$ (P7); $300 \mu \mathrm{m}$ and $20 \mu \mathrm{m}, n=4$ (4-week-old) mice. e, Percentage of intimal tdTomato ${ }^{+} \mathrm{CD} 45^{+}$cells in aortae of $\mathrm{Cx} 3 \mathrm{cr}$ TreERT2 $^{\text {CR26 }} 6^{\text {tdTomato }}$ mice at P7 and 4 weeks when labeled with tamoxifen postnatally ( $n=4$ (4-week-old), $n=5$ (P7) mice; Mann-Whitney t-test, $P=0.0159$, two tailed, mean \pm s.d., ${ }^{\star} P \leq 0.05$ ). f, Flow cytometry plots of tdTomato labeling of circulating cells at P7 and 4 weeks ( $n=3$ (4-week-old), $n=4$ (P7) mice). $\mathbf{g}$, Experimental design for the clonal tracing model and possible outcomes. Tamoxifen injection of $\mathrm{C} \times 3 \mathrm{cr} 7_{\text {CreERT2 }}$;R26 Rainbow mice at P1, P3 and P5. Aortae were collected at 8 weeks. Cer., Cerulean; mOr., mOrange; mCh., mCherry. h, Clones of Mac AIR cells labeled at 8 weeks (initial tamoxifen injections at P1, P3 and P5). CD45 cells are shown in white. Scale bar, $100 \mu$ m; $n=5$ mice. i, Intimal CD $11 \mathrm{c}^{+} \mathrm{CD} 45^{+}$cells from control and Ccr2-/- mice at P5 (top, former ductus arteriosus region) and 8 weeks (bottom, lesser curvature). CD11c, red; CD45, green; ERG, white. Scale bar, $100 \mu \mathrm{m}$ and $50 \mu \mathrm{m} ; n=7$ mice per time point. $\mathbf{j}$, Comparison of CD11 ${ }^{+} \mathrm{CD} 45^{+}$cells in aortae from control and Ccr2 ${ }^{-/-}$mice at P5 ( $n=6$ (control), $n=7$ ( $\left(\mathrm{cr} 2^{-/-}\right.$) mice; Mann-Whitney $t$-test, mean \pm s.d., $P>0.9999$ (exact), two tailed) or in aortae from mice at 8 weeks ( $n=7$ (control) or $n=6\left(C c r 2^{-/-}\right.$) mice; Mann-Whitney t-test, mean \pm s.d., $P=0.0872$, two tailed). NS, not significant. k, Summary of Figs. 1-3. Mac $^{\text {AIR }}$ cells enter the aorta via the ductus arteriosus, expand through self-renewal and populate the lesser curvature. Macrophages also accumulate in descending aortae from aged mice. Illustrations (a,b,g) were created using https://biorender.com/. 
whether monocytes participated in replenishment of Mac ${ }^{\text {AIR }}$ cells. Although the labeling frequency of $\mathrm{Mac}^{\mathrm{AIR}}$ cells decreased by $10 \% 10$ weeks after tamoxifen injection, we noted that reporter expression remained constant for an additional 10 weeks ( 20 weeks in total) (Extended Data Fig. 6e,h). This finding supports the conclusion of local self-renewal with negligible contribution from monocytes.
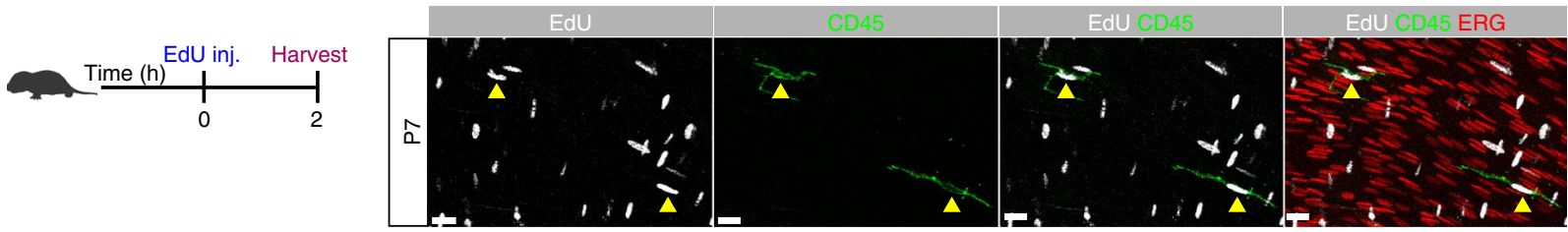

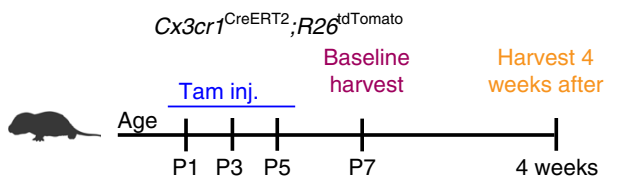

c 4 weeks

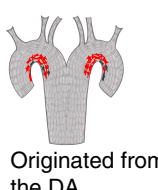

P7

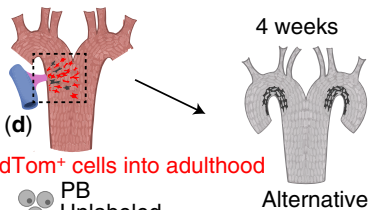

d
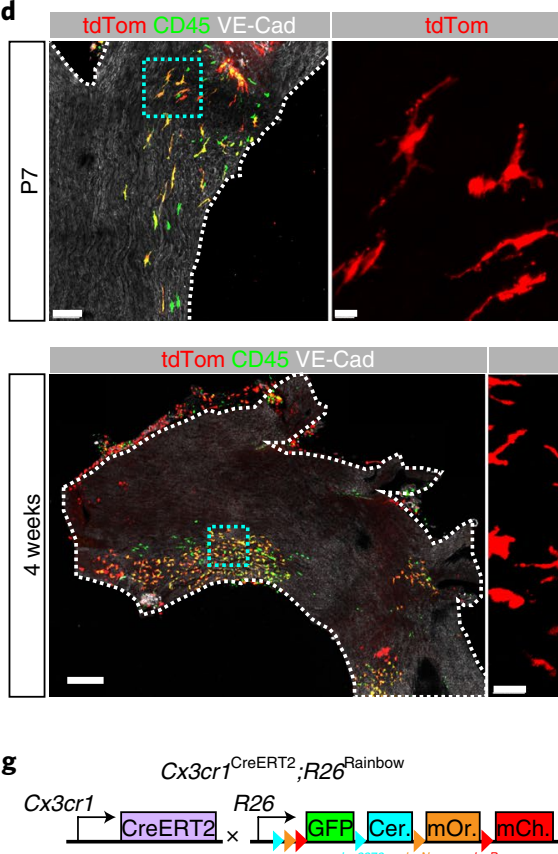

Age $\underset{\text { P1 P3 P5 }}{\left.\stackrel{\text { Tam inj. }}{\mid}\right|_{8 \text { weeks }} ^{\text {Harvest }}}$

Clonal expansion a alternative source

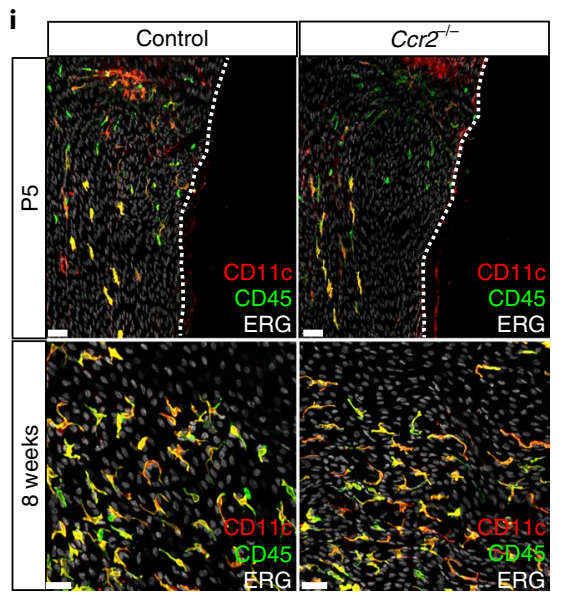

h

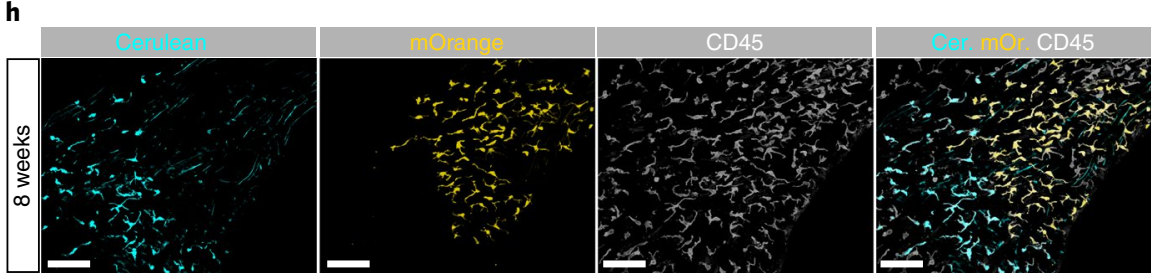

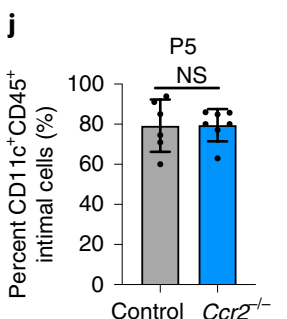

Control $\mathrm{Ccr}$

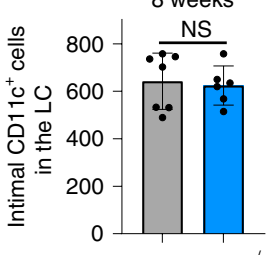

Control $\mathrm{Ccr}^{-1}$ k

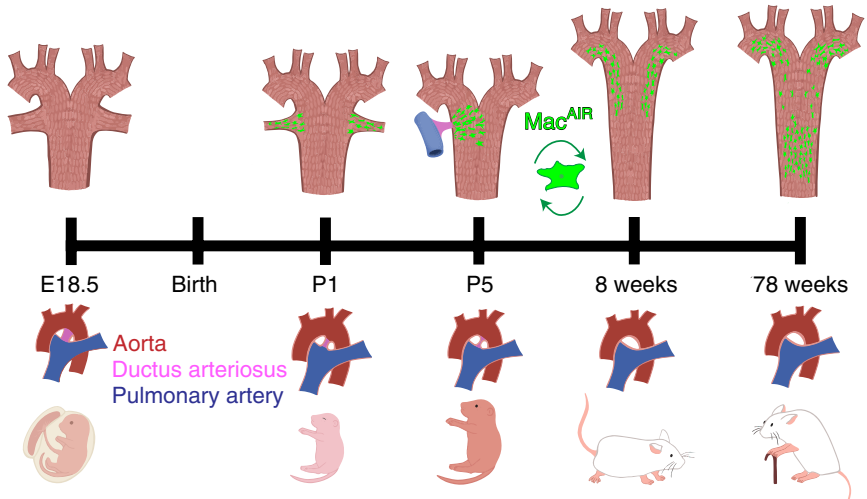


To further confirm these findings, we performed parabiosis experiments using adult $\mathrm{GFP}^{+}$and WT mice in which both mice shared chimeric circulation for 5 weeks (Extended Data Fig. 6i). The experiment allowed all circulating cells to access the aortic regions of interest (lesser curvature). The presence of $\mathrm{GFP}^{+}$intimal immune cells incorporated in WT mice would infer monocyte contribution and vice versa (Extended Data Fig. 6i). Only mice with excellent level of parabiosis were used (Extended Data Fig. 6j). Evaluation of the lesser curvature in WT mice revealed no $\mathrm{GFP}^{+}$intimal immune cells (Extended Data Fig. 6k), proving further strength to the conclusion that $\mathrm{Mac}^{\mathrm{AIR}}$ cell maintenance was independent of monocytes. This was also supported by clonal analysis of $C \times 3 \mathrm{cr} 1^{\text {CreERT2}}$; $R 26^{\text {Rainbow }}$ mice in which inducible Cre recombinase in cells expressing $C \times 3 \mathrm{cr} 1$ would randomly recombine, generating three fluorescently labeled populations (Extended Data Fig. 61). The data strongly support the notion that $\mathrm{Mac}^{\mathrm{AIR}}$ cells are self-maintained.

Mac $^{\text {AIR }}$ cells originate from definitive hematopoietic cells and expand through self-renewal. Mac ${ }^{\text {AIR }}$ cells colonize the aorta as they exit from the ductus arteriosus and migrate to areas of disturbed flow. After this initial wave of migration, Mac ${ }^{\text {AIR }}$ cells expand in number by local proliferation (Fig. 3a). Nonetheless, lineage tracing was required to fully ascertain adult progeny. Thus, we performed lineage analysis using the inducible $C \times 3 \mathrm{cr} 1^{\text {CreERT2}} ; R 26^{\text {dTomato }}$ reporter model to label $\mathrm{Mac}^{\mathrm{AIR}}$ cells immediately after birth and follow descendants over time (Fig. 3b,c). After administration of tamoxifen at birth, $63 \%$ of intimal CD $45^{+}$cells were positive for tdTomato by P7. At 4 weeks, $91 \%$ of intimal $\mathrm{CD} 45^{+}$cells in aortae retained tdTomato labeling (Fig. 3d-f). We interpret the increase from P7 $(63 \%)$ to 4 weeks $(91 \%)$ to indicate that only those inflammatory cells expressing $\mathrm{Cx} 3 \mathrm{cr} 1$ were successfully retained in the endothelium. Importantly, at 4 weeks, less than $0.5 \%$ of peripheral blood cells were positive for tdTomato (Fig. 3f), supporting the absence of contributions from circulating cells. Therefore, these data indicate that the Mac ${ }^{\text {AIR }}$ cells that enter the aorta postnatally expand via self-renewal and colonize the lesser curvature with minimal (if any) input from circulating monocytes.

To assess clonal expansion of $\mathrm{Mac}^{\mathrm{AIR}}$ cells at early time points, we again used the inducible $C x 3 c r 1^{\text {CreERT2 }} ; R 26^{\text {Rainbow }}$ model (Fig. $3 g$ ). Transgenic pups were treated with tamoxifen immediately after birth to induce stochastic recombination, yielding permanent expression of one of three mutually exclusive fluorescent proteins: Cerulean, mOrange and mCherry (Fig. 3g). At 8 weeks of age, clones of labeled Mac ${ }^{\text {AIR }}$ cells were observed (Fig. 3h), confirming that $\mathrm{Mac}^{\mathrm{AIR}}$ cells self-expand in situ after seeding the aorta immediately after birth. We also examined Mac ${ }^{\mathrm{AIR}}$ cells in chemokine receptor 2 (CCR2)-deficient $\left(\mathrm{Ccr}^{-/-}\right)$mice, in which Ly6C ${ }^{\text {hi }}$ (classical) monocyte emigration from the bone marrow is defective ${ }^{29}$. Compared to control mice, we found no difference in the proportion and number of intimal CD11 $\mathrm{c}^{+} \mathrm{CD} 45^{+}\left(\mathrm{Mac}^{\mathrm{AIR}}\right)$ cells in the aortae of either young (P7) or adult (8-week-old) mice (Fig. 3i,j). This further solidifies the notion that postnatal aortic intimal macrophages self-expand and maintain their population independently of monocytes at steady state (Fig. 3k).

Assessment of whether Mac ${ }^{\text {AIR }}$ cells were derived from primitive or definitive hematopoietic lineages was determined through experiments with a defined lineage-tracing model $\left(F l t 3^{\mathrm{Cre}} ; R 26^{\mathrm{mTmG}}\right)$ that labels cells arising from definitive hematopoietic stem cells as $\mathrm{GFP}^{+}$ (refs. ${ }^{27,30,31}$ ) (Extended Data Fig. 6m). Aortae from Flt3 ${ }^{\mathrm{Cre}} ;$ R2 $^{\mathrm{mTmG}}$ mice at P5 showed that a large proportion of intimal $C D 45^{+}$ cells were $\mathrm{GFP}^{+}$relative to circulating $\mathrm{CD} 11 \mathrm{~b}^{\text {hi }} \mathrm{Gr} 1^{\text {lo }}$ monocytes (Extended Data Fig. 6n,o). This labeling, combined with the observation that $\mathrm{Mac}^{\mathrm{AIRS}}$ were detected post-birth, strongly indicates that these cells were derived from a definitive hematopoietic lineage and not from the yolk sac, as is known to occur for some resident macrophage populations ${ }^{27,28}$.

$\mathrm{Mac}^{\mathrm{AIR}}$ cells blunt thrombin activity in areas of oscillatory flow. An inducible diphtheria toxin (DTx) model $\left(\mathrm{C} x 3 \mathrm{cr} 1^{\text {CreERT2}} ; \mathrm{Cs} f 1 r^{\mathrm{IIDTR}}\right)$ in which dual tamoxifen and DTx injections result in elimination of cells expressing both Cx3cr1 and Csf1r was used to deplete Mac ${ }^{\text {AIR }}$ cells and clarify their biological relevance (Fig. 4a). Efficient loss of Mac ${ }^{\text {AIR }}$ cells was noted $24 \mathrm{~h}$ after DTx injection (Fig. $4 \mathrm{~b}$ ) and was associated with altered endothelial morphology and apparent reduction in cell size (Fig. 4c and Extended Data Fig. 7a). To clarify whether cell size changes were due to cell contraction, we evaluated the expression of phosphorylated myosin light chain 2 (pMLC2). In control mice, Mac ${ }^{\text {AIR }}$ cells showed high expression of pMLC2 (Fig. 4d and Extended Data Fig. 7b). Upon Mac AIR depletion, endothelial cells became positive for PMCL2, indicating a highly contractile functional state, likely the reason behind the drastic change in cell size (Fig. 4c,d and Extended Data Fig. 7b). Together, these findings suggested that $\mathrm{Mac}^{\mathrm{AIR}}$ cells prevented a contractile endothelial phenotype that would otherwise manifest in areas of disturbed flow through an unclear mechanism.

It is well known that exposure of cultured endothelial cells to thrombin results in rapid Rho activation, phosphorylation of myosin light chain 2 (MLC2), stress fiber formation, contraction and disruption of endothelial junctions ${ }^{32,33}$ (Extended Data Fig. 7c). These events trigger downstream signaling by GPCR protease-activated

Fig. 4 | Mac ${ }^{A I R}$ cells are essential to prevent thrombin-mediated endothelial cell contraction in regions of oscillatory and disturbed flow. a, Transgenes

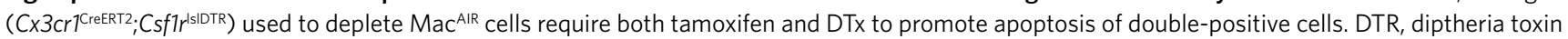

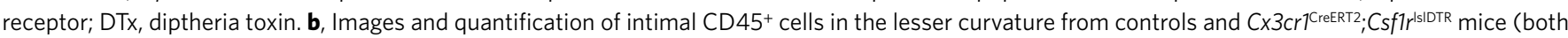
exposed for $24 \mathrm{~h}$ to tamoxifen and DTx). CD45+ (green) cells were effectively depleted. Scale bar, $50 \mu \mathrm{m} ; n=5$ mice per group, Mann-Whitney $t$-test, mean \pm s.d., $P=0.0079$, two tailed, ${ }^{\star \star} P \leq 0.01$. c, Surface area and elongation factor measurements of endothelial cells in the lesser curvature of $C \times 3 c r{ }^{c}$ ree

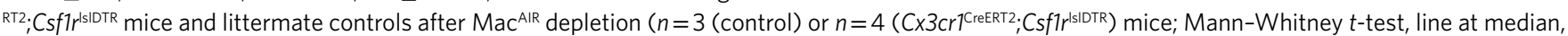
$P<0.0001$ (exact, surface area and elongation factor), two tailed, ${ }^{\star \star \star \star} P \leq 0.0001$ ). $\mathbf{d}, \mathrm{pMLC} 2$ staining (red) in the lesser curvature $1 \mathrm{~d}$ after tamoxifen and DTx injections of control and Cx3cr7reeRT2; Csf1ristitr mice. CD45, green; VE-Cad, white. Scale bar, $10 \mu \mathrm{m} ; n=5$ mice per group. e, Images of co-staining for MMP12 or MMP13 (red), CD45 (green) and VE-Cad (white). Note the colocalization of MMP12 and MMP13 with CD45+ cells. Scale bars, $20 \mu \mathrm{m}$ and $10 \mu \mathrm{m} ; n=3$ mice. f, Thrombin in areas of oscillatory and disturbed flow in aortae of 8-week-old C57BL/6 mice (IC, intercostal artery); scale bar, $20 \mu \mathrm{m}$;

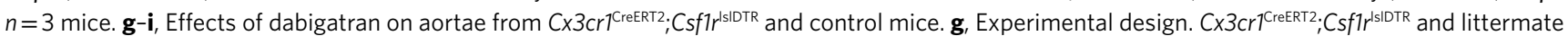
controls treated with tamoxifen and DTx for depletion of Mac ${ }^{A I R}$ cells. Vehicle or dabigatran was administered for $5 \mathrm{~d}$. The illustration was created using https://biorender.com/. h, Images of the lesser curvature $5 \mathrm{~d}$ after treatments as indicated. pMCL2, red; CD45, green; VE-Cad, white. Loss of pMLC2 in

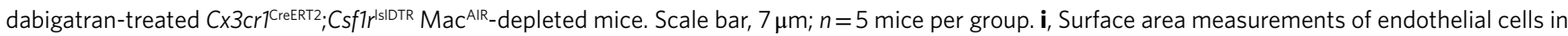
the lesser curvature of $\mathrm{C} \times 3 \mathrm{cr} 7^{\mathrm{CreERT2}} ; \mathrm{Cs} f \mathrm{r}^{\text {sIDTR }}$ mice and littermate controls $5 \mathrm{~d}$ after dabigatran treatment $(n=7$ mice per group, Mann-Whitney $t$-test, line at median, $P<0.0001$ (exact), two tailed, ${ }^{\star \star \star \star} P \leq 0.0001$ ). $\mathbf{j}$, Western blot of human aortic endothelial cells (HAECs) treated with thrombin or MMP13 or pretreated with MMP13 (time course), followed by thrombin treatment for an additional $5 \mathrm{~min}(n=4$ biological replicates, Mann-Whitney $t$-test, mean \pm s.d., $P=0.0286$ (vehicle versus thrombin; vehicle versus MMP13, 5 min; MMP13 versus thrombin; thrombin versus MMP13; thrombin versus MMP13, $1 \mathrm{~min}$; thrombin versus MMP13, 5 min), two tailed, $\left.{ }^{\star} P \leq 0.05\right)$. 
receptor (PAR)1, a thrombin receptor highly expressed by aortic endothelial cells (Extended Data Fig. 7d) and activated upon cleavage ${ }^{32,33}$. Interestingly, plasmin and other proteases, including MMP12 and MMP13 (both highly expressed by Mac ${ }^{\text {AIR }}$ cells) (Fig. 4e), can alter this signaling pathway ${ }^{34-37}$. Specifically, MMP13 can cleave PAR receptors on the carboxyl-terminal side of the thrombin cleavage site and thus prevent thrombin-mediated endothelial contraction ${ }^{34,36,37}$. Consequently, we posit that, in absence of Mac $^{\text {AIR }}$ cells, intact PAR1 could be activated by thrombin, leading to endothelial contraction. This assumption implied accumulation of thrombin in areas of disturbed flow. Indeed, immunocytochemistry revealed thrombin deposits at the lesser curvature and branch openings (Fig. 4f). Additionally, to further confirm thrombin activity in regions of disturbed flow, we treated control and $C \times 3 \mathrm{cr}$ $1^{\text {CreERT2 }}$; $s f 1 r^{\text {sIDTR }}$ mice with vehicle or dabigatran (thrombin inhibitor) and assessed endothelial cell contraction (Fig. 4g). Five days after treatment, dabigatran-treated control mice still expressed pMLC2 in Mac AIR cells, indicating that macrophage contraction was thrombin independent (Fig. 4h). However, depletion of Mac ${ }^{\text {AIR }}$ cells with concurrent dabigatran treatment prevented endothelial cell
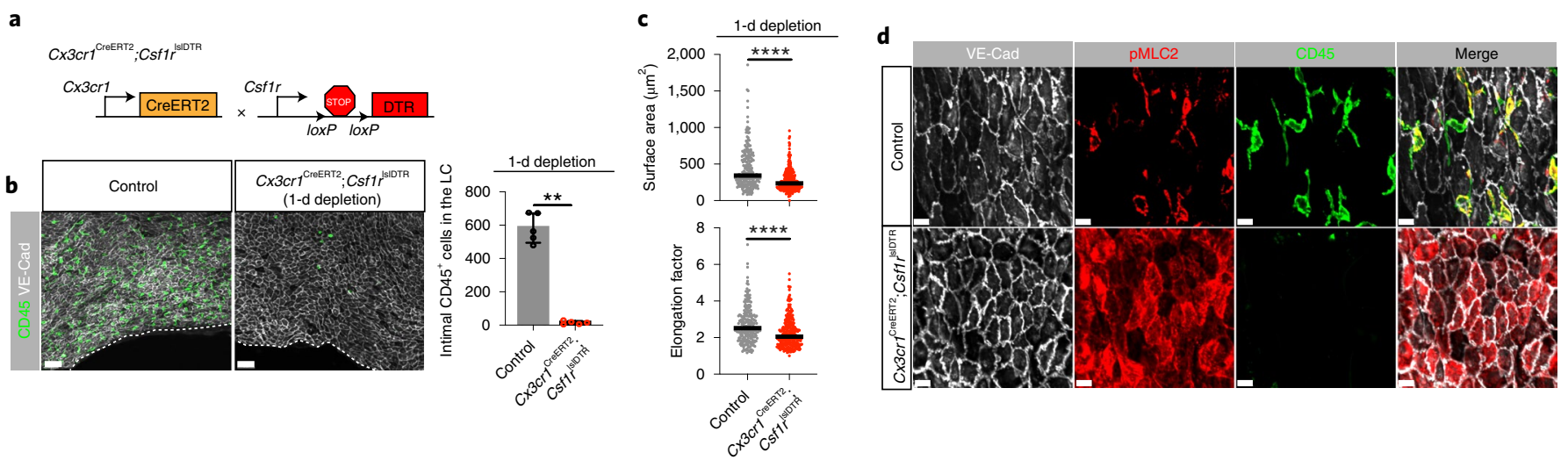

e

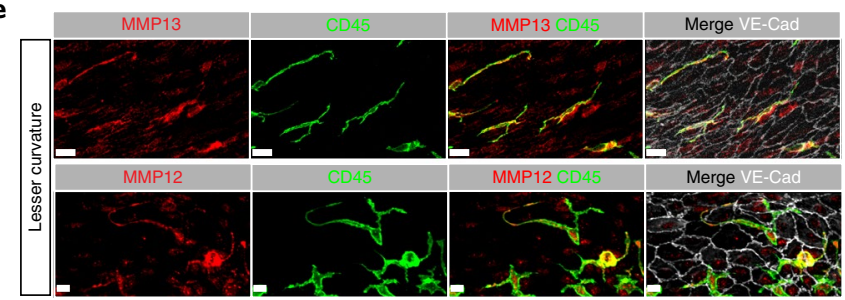

g

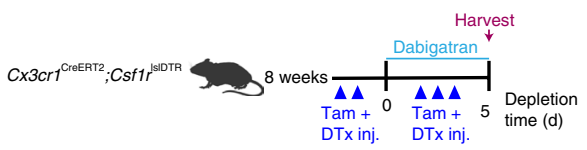

h

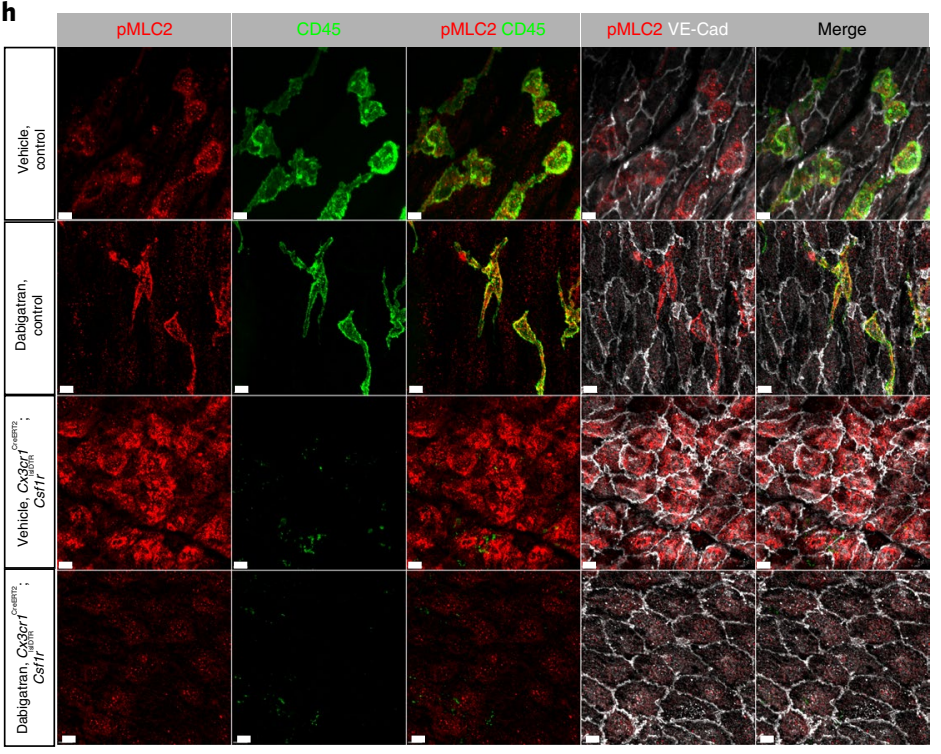

,
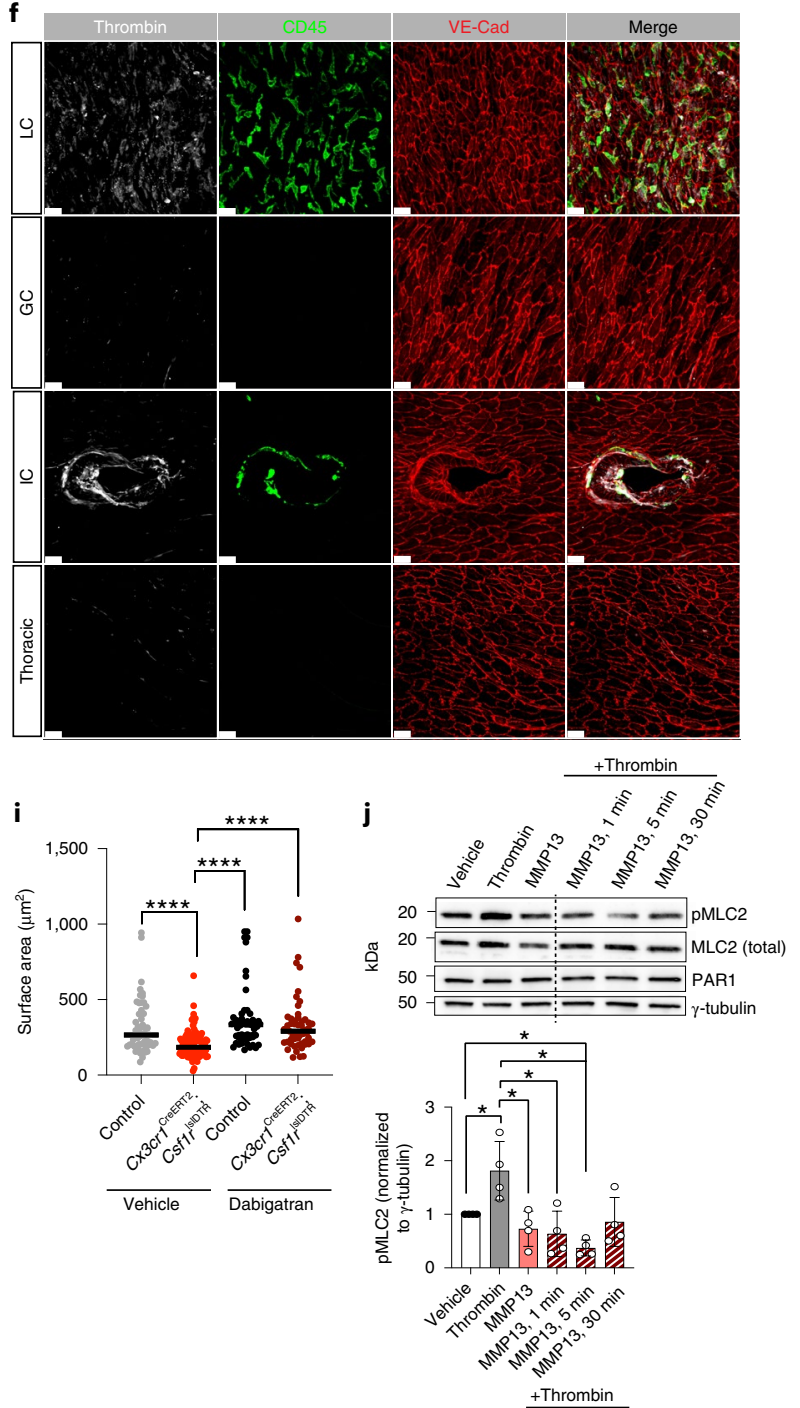
contraction, as shown by pMLC2 expression and cell size quantification (Fig. 4h,i), demonstrating that endothelial contraction was thrombin dependent but mitigated by Mac ${ }^{\text {AIR }}$ cells. Thrombin was still present in areas of oscillatory flow after macrophage depletion (Extended Data Fig. 7e). These findings indicate that Mac ${ }^{\text {AIR }}$ cells prevent endothelial contraction driven by thrombin in regions of disturbed flow. Importantly, we tested other aspects of clotting in the presence and absence of macrophages, including tail bleed times (Extended Data Fig. 7f) as well as other mediators or regulators of clotting such as nitric oxide and prostacyclin (Extended Data Fig. 7g,h). None of these were altered by depletion of $\mathrm{Mac}^{\text {AIR }}$ cells.

The next critical question was to sort out the molecular mechanism by which $\mathrm{Mac}^{\mathrm{AIR}}$ cells impaired thrombin action and, in particular, pMLC2. It is well established that thrombin mediates signals on endothelial cells through PAR1, PAR2 and PAR4) ${ }^{32,33}$, all expressed by aortic endothelial cells but not by Mac ${ }^{\text {AIR }}$ cells (Extended Data Fig. 7d). Interestingly, PAR receptors can be cleaved by thrombin, mediating signaling, but also by other proteases such as MMP12 and MMP13 blunting these signals by cleaving PAR1 on the carboxyl-terminal side of the thrombin site ${ }^{34-37}$. Considering the high levels of MMP12 and MMP13 expressed by Mac ${ }^{\text {AIR }}$ cells, it was only logical to predict that MMPs secreted by Mac ${ }^{\text {AIR }}$ cells impaired thrombin-mediated contraction. While testing this hypothesis in mouse aortae was impossible, we evaluated thrombin signaling, as shown by pMLC2 levels on cultured endothelial cells in the presence and absence of MMP13 (Fig. 4j). The findings showed that thrombin activates pMLC2 in the endothelium and that this effect is impaired by co-incubation with MMP13.

$\mathrm{Mac}^{\mathrm{AIR}}$ residency coincides with deposition of fibrin(ogen). The presence of thrombin in areas of disturbed flow led us to inquire whether fibrinogen, a thrombin substrate, was also found in these regions. Indeed, en face immunostaining revealed buildup of fibrin(ogen) in the lesser curvature (Fig. 5a). Higher magnification images (Fig. 5b) and 3D surface rendering (Fig. 5c) showed fibrin(ogen) decorating the surface of Mac ${ }^{\text {AIR }}$ cells. To confirm that fibrin(ogen) was deposited in regions of disturbed flow, we depleted endogenous fibrinogen in vivo and mice with fluorescently tagged fibrinogen to assess binding and accumulation (Fig. 5d). Knockdown of Fbg (fibrinogen gamma chain) was accomplished by delivery of small interfering (si)RNA targeting hepatic fibrinogen mRNA (siFibrinogen), which was encapsulated in lipid nanoparticles containing an ionizable cationic lipid ${ }^{38}$. Seven days after treatment, circulating fibrinogen was $\sim 90 \%$ depleted (Fig. 5e), and, at this time, fibrinogen-Alexa Fluor (A)488 (fbg-a488) avidly bound to $\mathrm{Mac}^{\mathrm{AIR}}$ cells and accumulated throughout the lesser curvature $3 \mathrm{~h}$ after injection (Fig. 5f). By contrast, fbg-a488 did not accumulate in the greater curvature (Extended Data Fig. 8a), confirming the predilection of fibrinogen for regions of disturbed flow.

We validated concurrent associations of $\mathrm{Mac}^{\mathrm{AIR}}$ cells and fibrin(ogen) in intercostal arterial openings (Fig. 5g). The correlation was also noted in descending aortae of 78-week-old mice (Extended Data Fig. 8b). Moreover, a time course evaluation showed progressive fibrin(ogen) accumulation in the aortic arch of mice at P7, 3 weeks and 8 weeks, coinciding with the age-dependent expansion of Mac $\mathrm{MIR}^{\mathrm{AR}}$ cells (Extended Data Fig. 8c). As fibrinogen is a substrate for macrophages, we predicted that fibrinogen deposits might be required for seeding $\mathrm{Mac}^{\mathrm{AIR}}$ cells in the regions of disturbed flow. Thus, we analyzed $\mathrm{Mac}^{\mathrm{AIR}}$ accumulation in fibrinogen-deficient mice $\left(\mathrm{Fbg}^{-/}\right)^{39}$ and in mice expressing a mutant form of fibrinogen that could not be converted to fibrin polymer $\left(F b g^{\mathrm{AEK}}\right)^{40}$. Our prediction was incorrect, as no difference in the abundance or distribution of $\mathrm{Mac}^{\mathrm{AIR}}$ cells was found in either mouse model (Fig. 5h). These findings revealed that, while overlapping in location, fibrin(ogen) was dispensable for seeding or anchoring $\mathrm{Mac}^{\mathrm{AIR}}$ cells to the tunica intima.

Mac $^{\text {AIR }}$ cells are necessary to clear fibrin(ogen) deposits in regions of disturbed flow. The concurrent presence of both thrombin and fibrin(ogen) in areas of disturbed flow implied that Mac ${ }^{\text {AIR }}$ cells might be involved in preventing fibrin formation; therefore, we explored fibrin(ogen) accumulation in the $C x 3 \mathrm{cr} 1^{\mathrm{CrERT} 2} ; \mathrm{Cs} f 1 r^{\mathrm{slDTR}}$ model over time (Fig. 6a). Evaluation of fibrin(ogen) levels at 7 and $14 \mathrm{~d}$ after continued depletion showed progressive and significant accumulation in the lesser curvature (Fig. 6b-d and Extended Data Fig. 8d). Additionally, fibrin fibrils, which were not detected in any control mice in our experiments, were clearly visible in the aortae of mice depleted of macrophages for $14 \mathrm{~d}$ (Fig. $6 \mathrm{c}$ ). The data imply that $\mathrm{Mac}^{\mathrm{AIR}}$ cells promote clearance of fibrin(ogen) and/or prevention of fibrin formation. Further, en face SEM images of aortae (Fig. 6e and Extended Data Fig. 8e) revealed microthrombi and polymerized fibrin decorating the lesser curvature of $C x 3 c r 1^{\text {CreERT2 }} ; C s f 1 R r^{\text {sIDTR }}$ mice but not present in littermate controls. Microthrombi were also noted by confocal microscopy along with rupture of the endothelial lining (Fig. 6f,g). As a direct readout of disseminated microthrombi, we evaluated $\mathrm{D}$-dimer levels and found markedly elevated levels in macrophage-depleted mice compared to undetectable levels in control mice (Fig. 6h), further supporting hemostatic imbalance. Histological examination of tissues from $\mathrm{Mac}^{\mathrm{AIR}}$-depleted mice revealed hemorrhagic foci in multiple tissues, including the kidney, liver and lung (Fig. 6i). Images of kidneys from $C \times 3 c r 1^{\text {CreERT2}} ; C s f 1 r^{1}$ sIDTR mice that had to be euthanized due to health decline exhibited abundant fibrin(ogen) throughout the tissue (Fig. 6j), consistent with vascular rupture. Based on the data presented, it is likely that dislodged microthrombi traveling through the circulatory system were responsible for occluding smaller-diameter vessels, leading

Fig. 5 | Fibrin(ogen) is deposited and binds to Mac ${ }^{\text {AlR }}$ cells in regions that experience oscillatory and disturbed flow. a, Whole-mount, en face images of fibrin(ogen) (white) and CD45+ cells (green) in the aortic arch of 8 -week-old C57BL/6 mice. Scale bars, $500 \mu \mathrm{m}$ and $50 \mu \mathrm{m} ; n=20$ mice. $\mathbf{b}$, Fibrin(ogen) (white) was overlaid with CD45 staining (green) in the adult aortic arch. Higher magnification (bottom) of blue dotted inserts highlights fibrin(ogen) staining in an area that resembles the cellular process of a Mac AlR cell (blue dotted ellipse). Scale bars, $10 \mu M$ and $5 \mu M$; $n=20$ mice. $\mathbf{c}$, En face images of fibrin(ogen) location in the aortic arch displayed using maximum-intensity projections (top) and 3D surface rendering (bottom). Scale bars, $7 \mu \mathrm{m}$ and $4 \mu \mathrm{m} ; n=16$ mice. d, Experimental design. Eight-week-old C57BL/6 mice were injected via the tail vein with either siFibrinogen (siFbg) or siLuciferase (siluc) (control). Seven days later, mice were injected via the tail vein with fbg-a488. Three hours after fbg-a488 injection, aortae were collected for analysis. The illustration was created using https://biorender.com/. e, Plasma levels of fibrinogen $7 \mathrm{~d}$ after mRNA injection, measured by ELISA ( $n=4$ mice per group, Mann-Whitney $t$-test, mean \pm s.d., $P=0.0286$, two tailed, $\left.{ }^{\star} P \leq 0.05\right)$. $\mathbf{f}$, Distribution of intravenously injected fbg-a488 in relation to Mac ${ }^{A l R}$ cells. CD45 is shown in white. Yellow arrowheads indicate Mac ${ }^{A I R}$ surface-bound fbg-a 488 ( $n=4$ mice per group; scale bar, $4 \mu \mathrm{m}$ ). $\mathbf{g}$, Intercostal artery from adult (8-week-old) mice exhibiting fibrin(ogen) deposition (white) and intimal CD45+ cell accumulation (green). VE-Cad (red) identifies endothelial cells (scale bar, $70 \mu \mathrm{m}, n=15$ mice). $\mathbf{h}$, En face images of the aortic arch comparing fibrin(ogen) accumulation (green) and immune cell (CD45, white) deposition in control (h(i); $n=8$ mice), Fbg $^{-/-}$(h(ii); $n=5$ mice) and Fbg ${ }^{\text {AEK }}$ (h(iii); $n=6$ mice) mice. VE-Cad identifies endothelial cells in red. Scale bars, $300 \mu \mathrm{m}$ and $8 \mu \mathrm{m}$. The adjacent graph represents quantification of Mac ${ }^{\text {AIR }}$ cells in the lesser curvature of the aortic arch in Fbg ${ }^{-/-}$and Fbg ${ }^{\text {AEK }}$ mice compared to colony controls $\left(n=3\left(\mathrm{Fbg}^{\mathrm{WT} / \mathrm{WT}}\right), n=5\left(\mathrm{Fbg}^{-/-}\right), n=5\left(\mathrm{Fbg}^{\mathrm{WT} / \mathrm{WT}}\right), n=6\right.$ (Fbg $\left.{ }^{\mathrm{AEK}}\right)$ mice per group; Mann-Whitney $t$-test, mean $\pm \mathrm{s} . \mathrm{d}$., $P=0.3929$, 
to hemorrhagic foci and organ damage. Additional support for the requirement of $\mathrm{Mac}^{\mathrm{AIR}}$ cells in clearing fibrin(ogen) deposits also emerged from evaluation of $C d 11 c^{-/-}$mice. The absence of CD11c resulted in a significant reduction in $\mathrm{Mac}^{\mathrm{AIR}}$ numbers, indicating that that CD11c was necessary, albeit not fully sufficient, for anchorage of $\mathrm{Mac}^{\mathrm{AIR}}$ cells to the intimal niche, as we could still detect some macrophages (approximately 45\% in comparison to controls) in the tunica intima of this mouse. Importantly, this mouse model also exhibited an impressive accumulation of fibrin(ogen) (Extended Data Fig. 8f).

An alternative explanation for fibrin(ogen) accumulation and thrombosis upon Mac AIR depletion could relate to breach of
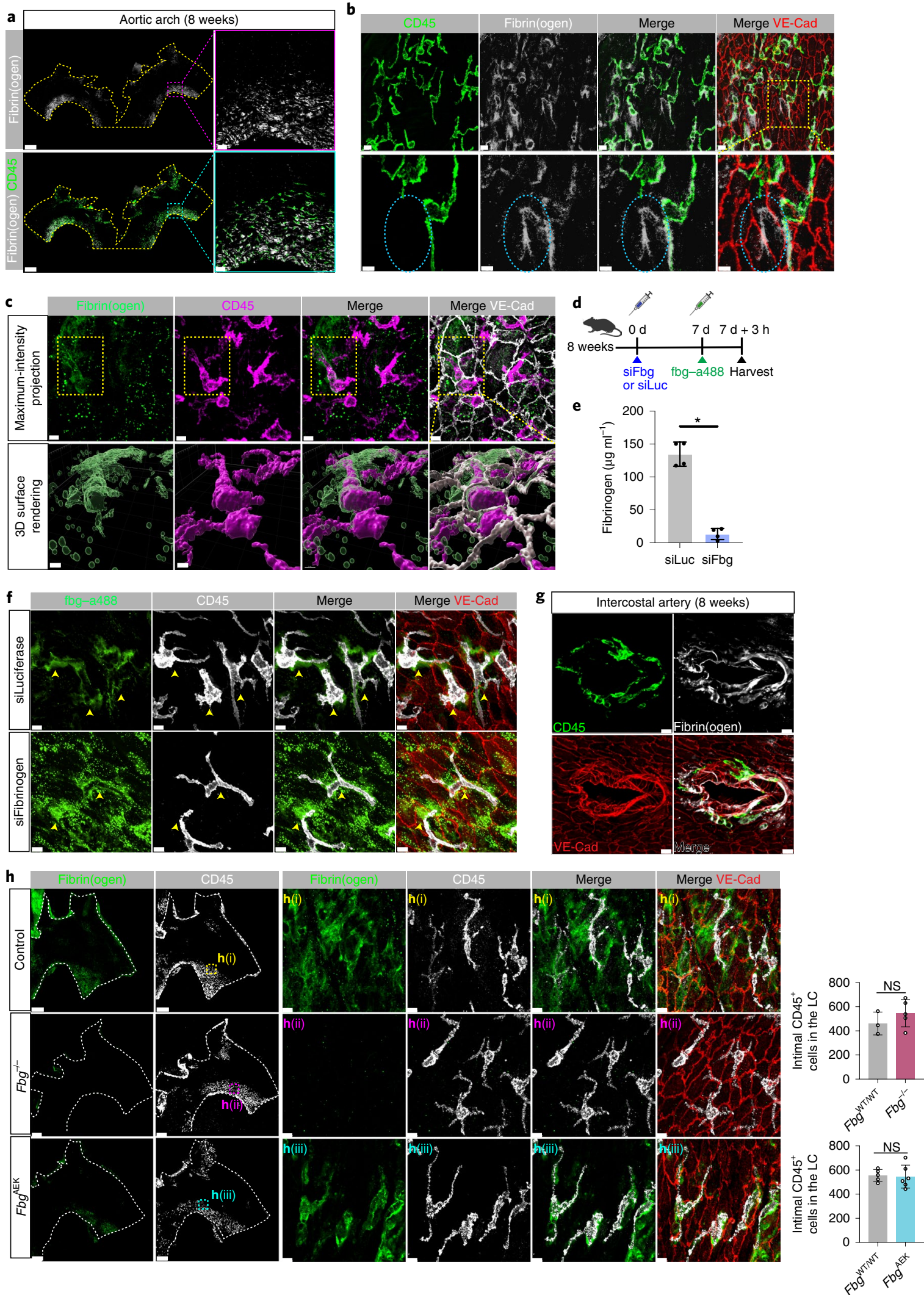
barrier integrity in areas of oscillatory flow, implicating Mac ${ }^{\text {AIR }}$ cells in the maintenance of endothelial junctional integrity in these areas. We tested this possibility by examining fluorescent microsphere $(40 \mathrm{~nm})$ deposition in the tunica intima of littermate control and $C \times 3 c r 1^{\text {CreERT2}} ; C s f 1 r^{\text {slDTR }}$ mice that both received tamoxifen and DTx injections ( $1 \mathrm{~d}$ of Mac ${ }^{\text {AIR }}$ depletion) (Extended Data Fig. 8g). While the positive control (buffered EDTA intracardiac injection for $5 \mathrm{~min}$ ) resulted in robust deposition of fluorescent beads in between cells, no accumulation of fluorescent beads was found in any of the other groups (Extended Data Fig. 8g). Thus, Mac ${ }^{\text {AIR }}$ cells do not play a role in maintaining barrier integrity; instead, they appear to be necessary to clear fibrin(ogen) deposits and mitigate PAR1-thrombin signaling in regions of disturbed flow.

The drastic phenotype observed from depletion of Mac ${ }^{\text {AIR }}$ cells, a relatively small population, prompted the question of whether the approach might affect a broader group of macrophages. Thus, we evaluated alterations in the macrophage populations of multiple organs by flow cytometry analysis (Fig. 6k). The findings revealed that, while bone marrow and peripheral blood were affected by the dose of tamoxifen and DTx used, none of the other CX3CR $1^{+} \mathrm{CSF} 1 \mathrm{R}^{+}$ macrophage populations in the evaluated organs were altered. The implication is that areas of oscillatory and disturbed flow in the arterial tree are sites of fibrin accumulation that absolutely depend on $\mathrm{Mac}^{\mathrm{AIR}}$ cells for clearance with critical consequences. A few last important pieces of evidence to solidify this conclusion are still pending, including how $\mathrm{Mac}^{\mathrm{AIR}}$ cells degrade fibrin(ogen).

Macrophages are known to express plasminogen receptors to degrade fibrin(ogen) extracellularly ${ }^{41}$. Importantly, Mac ${ }^{\text {AIR }}$ cells expressed plasminogen receptors (Fig. 6l) and bound to fluorescently conjugated plasminogen when injected intravenously (Fig. $6 \mathrm{~m}$ ). Thus, in addition to MMP12 and MMP13, Mac ${ }^{\text {AIR }}$ cells are capable of generating cell surface-associated plasmin, particularly with the aid of endothelial cells, which express high levels of tissue-type plasminogen activator (Extended Data Fig. 8h,i). We also confirmed that neither endothelial cells nor Mac ${ }^{\text {AIR }}$ cells expressed plasminogen (Extended Data Fig. 8j). These findings indicate that Mac ${ }^{\mathrm{AIR}}$ cells are required to clear fibrin(ogen) deposits, prevent fibrin formation and maintain an anti-thrombotic state in areas of disturbed flow.

Additional support for the conclusion that $\mathrm{Mac}^{\mathrm{AIR}}$ cells are responsible for clearing fibrin(ogen) deposits in regions of disturbed flow came from experiments in which Mac ${ }^{\mathrm{AIR}}$ cells were allowed to return after a 2-week depletion. Much like resident macrophages in other organs ${ }^{27,28,42-44}$, we found that, upon removal of Mac $\mathrm{MIR}^{\mathrm{AIR}}$ cells and elimination of depletion pressure (tamoxifen and DTx), monocytes seeded areas of disturbed flow and reconstituted the Mac AIR population (Fig. $7 \mathrm{a}-\mathrm{d}$ ). A gradual increase in macrophage number was noted 1 and 2 weeks after depletion (Fig. $7 \mathrm{~d}$ ). Importantly, the increase in $\mathrm{Mac}^{\text {AIR }}$ numbers was associated with a reduction of accumulated fibrin(ogen) (Fig. 7c,e). Moreover, circulating D-dimer levels (Fig. 7f) and endothelial cell size in the lesser curvature of the aorta (Fig. 7g) also returned to control levels, demonstrating full rescue of the phenotype. Overall, these findings further support the conclusion that Mac ${ }^{\text {AIR }}$ cells are required to clear fibrin(ogen) deposits in regions of disturbed flow.

\section{Discussion}

Vascular endothelial and hematopoietic cells are well known to coordinate inflammatory responses. Here, we have expanded these functions to also include regulation of intravascular hemostasis. Indeed, our findings indicate that, while endothelial cells provide a non-thrombogenic surface, facilitating blood circulation in areas of disturbed flow, this function is challenged by the accumulation of fibrinogen and thrombin (aortic arch and branches with rapid flow). In these regions, the presence of a population of intima resident macrophages ( $\mathrm{Mac}^{\mathrm{AIR}}$ ), summoned to areas of disturbed flow from birth, is critical to effectively clear fibrinogen and prevent intravascular clotting.

Macrophage association with the endothelium is not necessarily surprising, and, when seen in aortic tissue sections, the assumption is that this heterotypic interaction might be part of an inflammatory or pre-atherosclerotic lesion. However, the unusual feature that captured our attention was that seeding of this macrophage population occurred after birth; furthermore, these cells displayed a unique topology in relation to the endothelium. These elements indicated that the presence of these macrophages in the luminal aspect of the aorta was not part of an inflammatory response; instead, the process was a normal developmental program by which Mac ${ }^{\text {AIR }}$ cells become a constitutive component of the tunica intima in regions of disturbed flow. While here we addressed multiple points related to origin, lineage and self-renewal, questions such as why these cells are attracted to areas of disturbed and oscillatory flow and how and why do they migrate from the closing ductus arteriosus to these regions remain unanswered. A logical assumption is that disturbed flow alters the endothelium, creating unique niche conditions that attract monocytes to these sites and promote $\mathrm{Mac}^{\mathrm{AIR}}$ differentiation into a stable population capable of self-renewal.

The ability of macrophages to cleave fibrinogen and participate in the remodeling of the provisional matrix formed during wound

Fig. 6 | Mac AIR cells are necessary to clear fibrin deposits in regions of oscillatory and disturbed blood flow. a, Experimental design for long-term Mac AIR depletion. Cx3cr7 ${ }^{\text {CreERT2 }}$; Csf1ristDTR mice and littermate controls were injected every other day, alternating between tamoxifen and DTx. $\mathbf{b}$, Accumulation of fibrin(ogen) (green) in the lesser curvature $14 \mathrm{~d}$ after Mac ${ }^{A I R}$ depletion in Cx3cr7CreERT2; Csf1rlsidTR mice compared to in control mice (scale bars, $500 \mu \mathrm{m}$ (left) and $150 \mu \mathrm{m}$ (right); $n=4$ (control), $n=11$ (CX3cr7 ${ }^{\text {CreERT2; Csf1rIIDTR }) ~ m i c e ~ p e r ~ g r o u p . ~ c, ~ F i b r i n ~ f i b r i l s ~(g r e e n) ~ a f t e r ~ M a c ~}{ }^{\text {AIR }}$ depletion (14 d) (yellow dotted ovals). VE-Cad, white; CD45, pink (scale bar, $10 \mu \mathrm{m} ; n=4$ (control), $n=11$ (Cx3cr7 CreERT2;Csf1ristR) mice per group). Fb(g), fibrin(ogen). d, Mean fluorescence intensity (MFI) of fibrin(ogen) in the lesser curvature of Cx3cr7CreERT2; Csf1rlsiDTR and control mice $14 \mathrm{~d}$ after depletion ( $n=4$ mice per group, Mann-Whitney $t$-test, mean \pm s.d., $P=0.0286$, two tailed, ${ }^{\star} P \leq 0.05$ ). AU, arbitrary units. e, SEM showing microthrombi in the lesser curvature of control and $C x 3 c r 7$ reeRT2; Csf1rsIDTR mice $14 \mathrm{~d}$ after depletion. Scale bars, $20 \mu \mathrm{m}$ and $5 \mu \mathrm{m} ; n=3$ mice per group. $\mathbf{f}$, Microthrombi (green) and intima ruptures (yellow arrowheads) in a Cx3cr7 ${ }_{\text {CreERT2 }}$ Csf1r IsIDTR mouse that was euthanized due to declined health $7 \mathrm{~d}$ after depletion. Scale bar, $20 \mu \mathrm{m}$. $\mathbf{g}$, Intima ruptures with high fibrinogen (yellow arrowheads) in CX3cr7reERT2; Csf1risIDTR mice $14 \mathrm{~d}$ after depletion (fibrinogen, green; VE-Cad, red). Scale bar, $20 \mu \mathrm{m} ; n=4$ mice. h, D-dimer plasma levels in

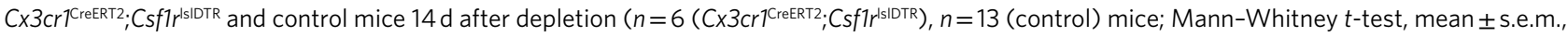
$P=0.0024$, two tailed, $\left.{ }^{\star \star} P \leq 0.01\right)$. i,j, Kidneys, lung and liver from a $C \times 3$ cr $^{\text {CreERT2}} ;$ Csf1rsidTR mouse with 7 or $14 \mathrm{~d}$ of macrophage depletion. $\mathbf{i}$, Multiple

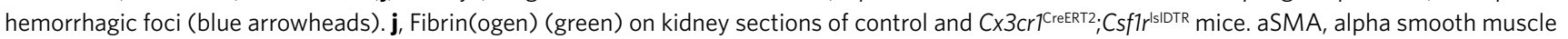

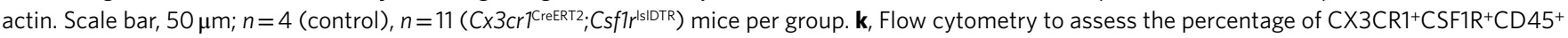
cells in bone marrow (BM), lung, liver, kidney and peripheral blood in control and $C \times 3 \mathrm{cr}^{\text {CreERT2}}{ }^{\text {; }}$ Csf1R ${ }^{\text {IIDTR }}$ mice $1 \mathrm{~d}$ after depletion ( $n=5$ mice per group ( $n=3$ mice, peripheral blood), Mann-Whitney $t$-test, mean \pm s.e.m.; $P=0.007937$ (bone marrow), $P=0.0065$ (peripheral blood); two tailed, ${ }^{\star \star} P \leq 0.01$ ). Q, quartile. I, Transcript levels of plasminogen receptors (Eno1 and Plgrkt) in adventitial macrophages (adv M $\varphi$ ) and Mac ${ }^{\text {AIR }}$ cells from scRNA-seq (Extended Data Fig. 2). m, Left, experimental strategy for intravenous injection of FITC-conjugated albumin or plasminogen. Right, distribution of injected FITC-conjugated albumin (control, top) or plasminogen (bottom) in relation to Mac ${ }^{\text {AIR }}$ cells (yellow arrowheads). CD45, white; PECAM1, red. Scale bar, $5 \mu \mathrm{m} ; n=3$ per group. The illustration was created using https://biorender.com/. 
healing has been established long ago ${ }^{45}$. However, the repertoire of proteases is distinct in different macrophage subtypes; some proteases are expressed only upon induction and in situations of wound healing. We found that, unlike adventitial and other macrophage populations, Mac ${ }^{\text {AIR }}$ cells constitutively express MMP12 and MMP13, known to cleave fibrinogen ${ }^{46-48}$. In addition, Mac ${ }^{\text {AIR }}$ cells also bind to plasminogen, and this anchorage enables endothelial tissue plasminogen activator (tPA) to generate plasmin, which also degrades fibrinogen and fibrin. Together, these proteases enable intravascular macrophages to efficiently remove fibrinogen deposits and antagonize fibrin accumulation driven by procoagulant pathways and the presence of thrombin in areas of oscillatory and disturbed flow.

Supporting the biological role attributed to $\mathrm{Mac}^{\mathrm{AIR}}$ cells, it is pertinent to remember that, while compatible with development and reproduction, plasminogen deficiency results in a severe thrombotic phenotype in both normal and inflamed tissues of adult animals ${ }^{39,49,50}$. Homozygous plasminogen-deficient mice display spontaneous
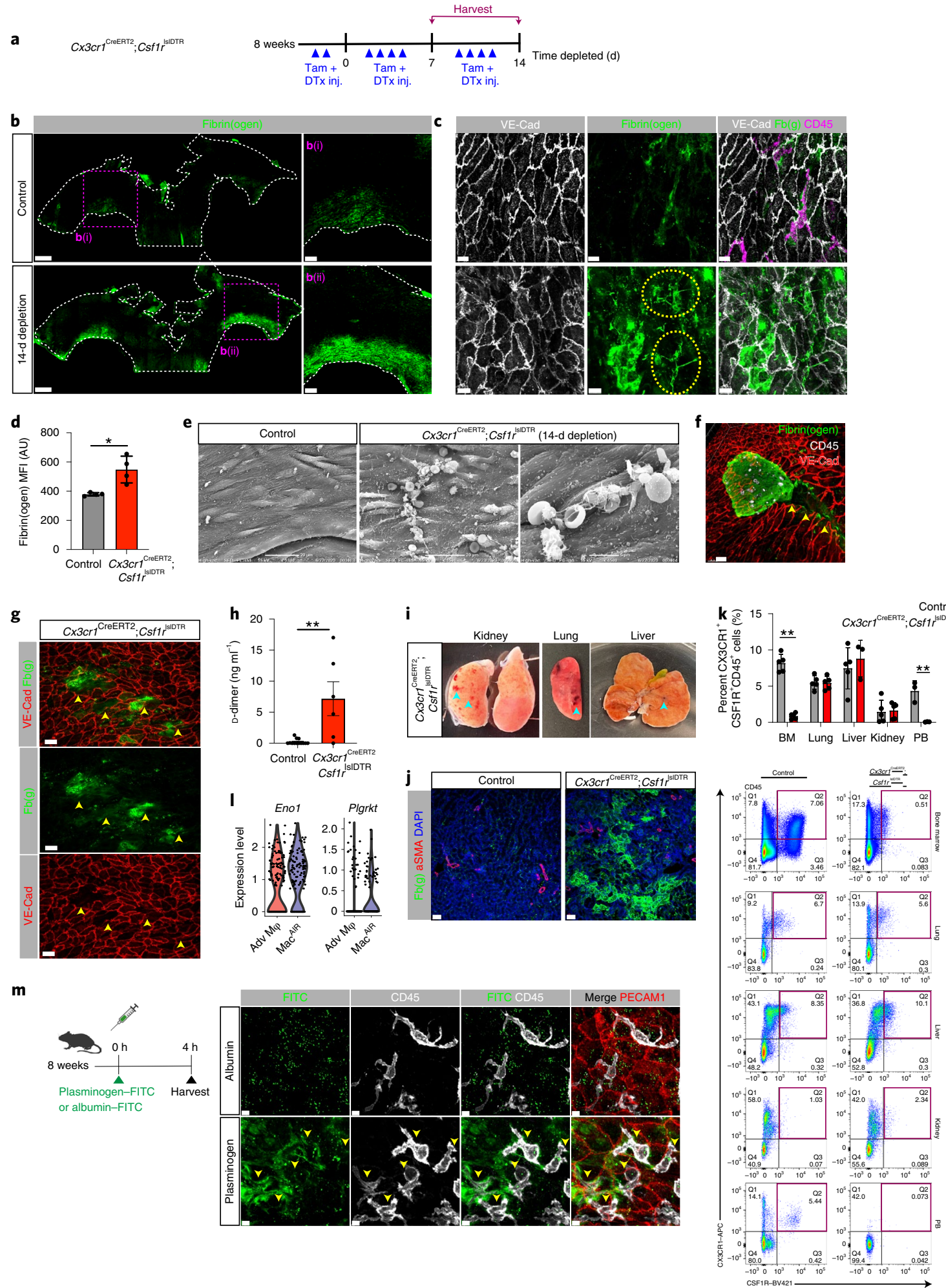

m

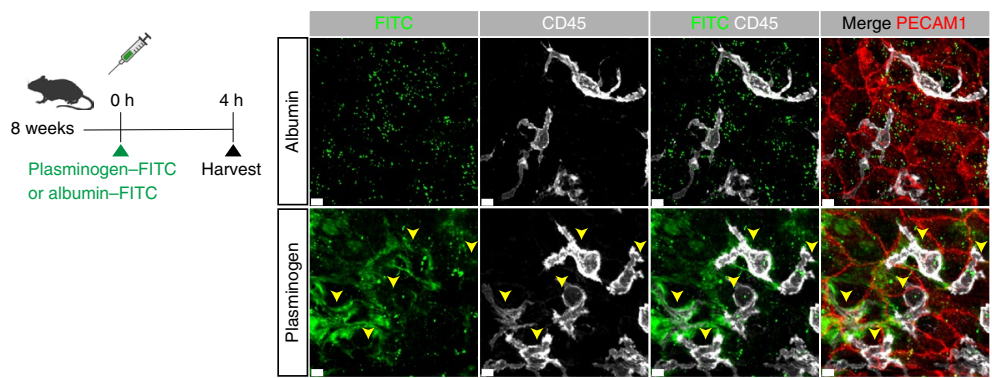



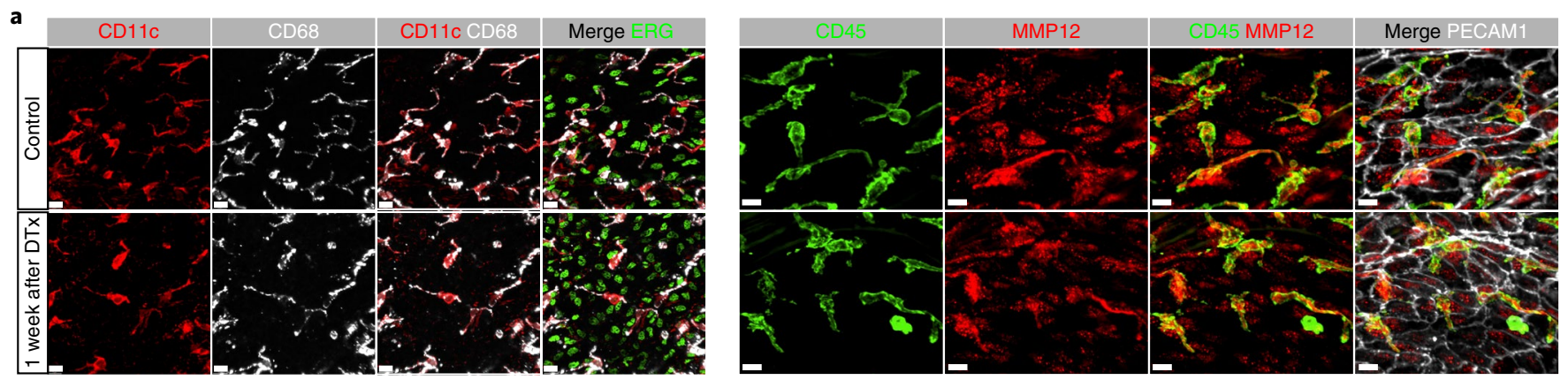

b

2 weeks after depletion

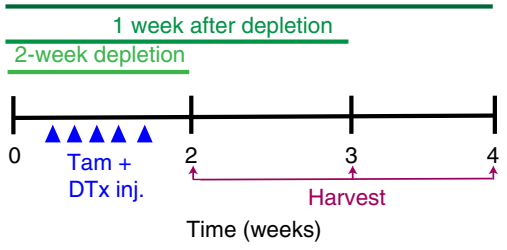

c

Cx3cr1 ${ }^{\text {CreERT2 }} ;$ CSF1 $^{\text {ISIDTR }}(10-12$ weeks old $)$
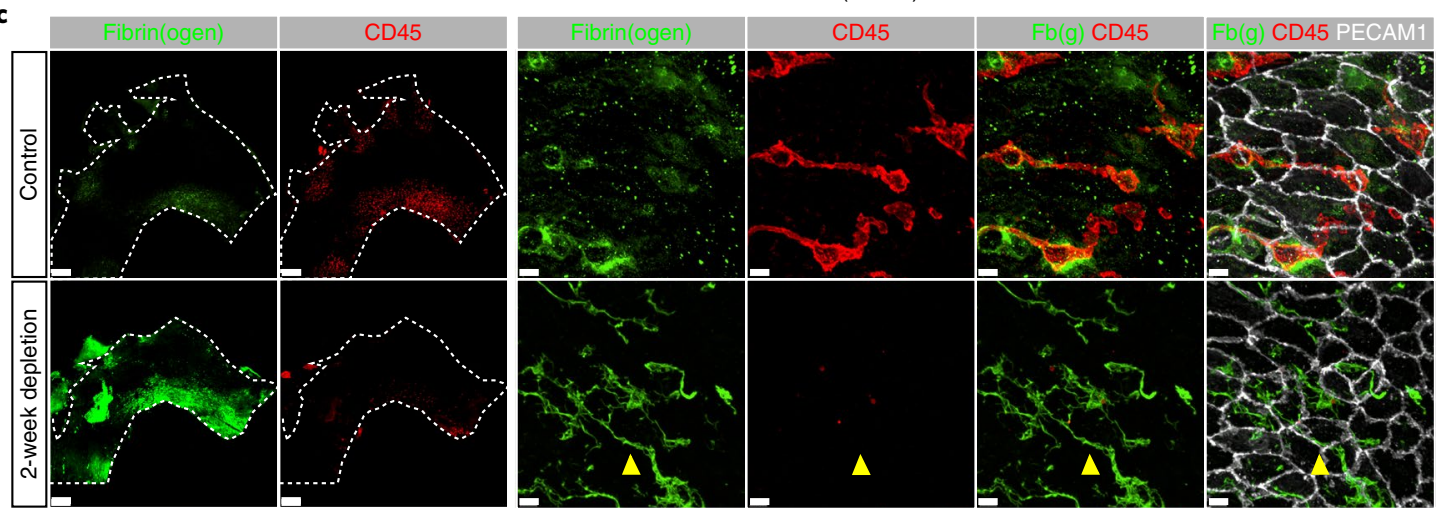
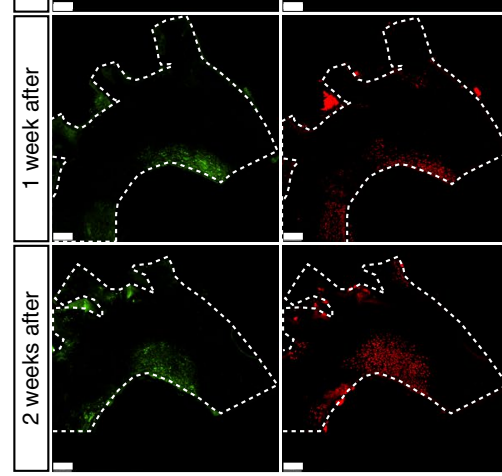

d

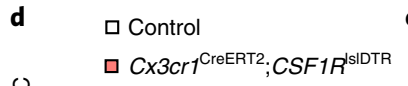

e

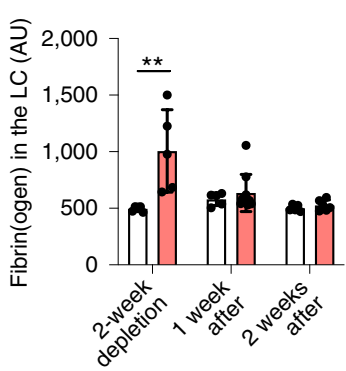

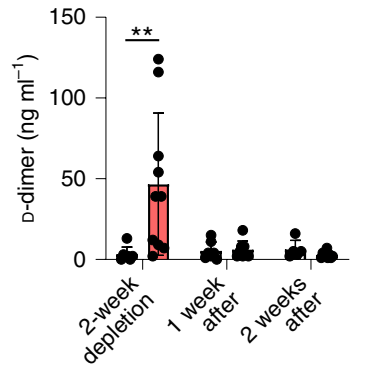

g

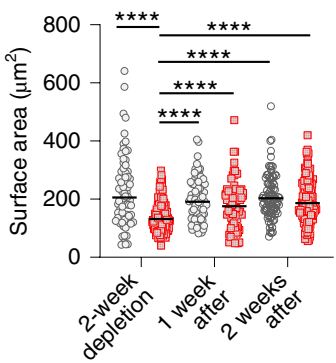

thrombotic lesions in multiple organs and a median survival of $176 \mathrm{~d}$, with about $40 \%$ of mice succumbing to death. Additionally, these death phenotypes are effectively reversed by the simultaneous imposition of fibrinogen deficiency ${ }^{39}$. These findings underlie a critical constitutive function of the plasminogen-activation system for fibrin surveillance and clearance in non-pathological settings and resonate extremely well with the findings described here.

Given the association of $\mathrm{Mac}^{\mathrm{AIR}}$ cells with areas of disturbed flow, also known to be pro-atherogenic sites, their potential contribution to atherosclerotic lesions is an important question. Specifically, one 


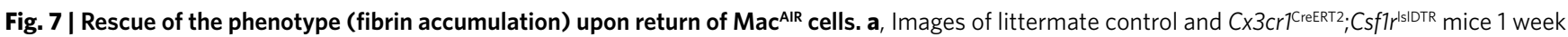
after a single DTx injection. Stopping tamoxifen and DTx injections relieves the pressure of cell ablation and allows for the repopulation of intimal immune cells. Aortae were stained for Mac ${ }^{\text {AIR }}$ markers to validate the monocyte-derived population that repopulates the arch after the resident population is depleted (Mac AlR identity, $\left.\mathrm{CD} 11 \mathrm{c}^{+} \mathrm{MMP12}{ }^{+} \mathrm{CD} 68^{+} \mathrm{CD} 45^{+}\right)(n=3$ mice per stain; scale bars, $15 \mu \mathrm{m}$ (left) and $10 \mu \mathrm{m}$ (right)). b, Schematic of macrophage

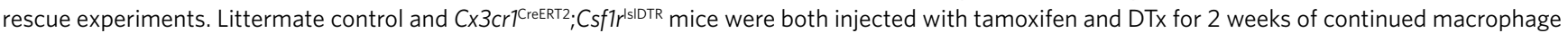
depletion. After 2 weeks, we stopped administration of tamoxifen and DTx, allowing Mac ${ }^{\text {AlR }}$ cells to repopulate the aorta. Aortae were evaluated en face at 2 weeks of depletion (baseline) and after ceasing tamoxifen or DTx treatment ( 1 week after depletion and 2 weeks after depletion). c, En face images evaluating fibrin(ogen) (green) and Mac ${ }^{\text {AIR }}$ (red) accumulation in aortae of littermate control and Cx3cr ${ }^{\text {CreERT2; }}$ Csf1rsiDTR mice at the given time points. Fibrin fibrils are indicated by yellow arrowheads. VE-Cad, white; 1 week after, 1 week after depletion; 2 weeks after, 2 weeks after depletion. Scale bars, $300 \mu \mathrm{m}$ and $7 \mu \mathrm{m} ; n=6$ mice per group. $\mathbf{d}$, Total number of intimal CD45+ cells in the lesser curvature of the aortic arch in littermate controls and Cx3cr7creeRT2; Csflristr mice at 2 weeks of depletion, 1 week after depletion and 2 weeks after depletion $(n=5$ (control, 1 week after), $n=6$ (DTR, 2 weeks after), $n=7$ mice per group; Mann-Whitney $t$-test, mean \pm s.d., $P=0.00116$ (2-week depletion), two tailed, ${ }^{\star \star} P \leq 0.01$ ). e, Mean fluorescence intensity of fibrin (ogen) in the lesser curvature of the aortic arch ( $n=5,6$ (DTR, 2 weeks after), $n=10$ (DTR, 1 week after) mice per group; Mann-Whitney $t$-test, mean \pm s.e.m., $P=0.007937$ (2-week depletion), two tailed, ${ }^{\star \star} P \leq 0.01$ ). f, D-dimer plasma levels ( $n=5$ (control, 2 weeks after), $n=7$ (control, 2-week depletion and 1 week after), $n=8$ (DTR, 1 week after), $n=9$ (DTR, 2 weeks after), $n=10$ (DTR, 1-week depletion) mice; Mann-Whitney $t$-test, mean \pm s.d., $P=0.0034$

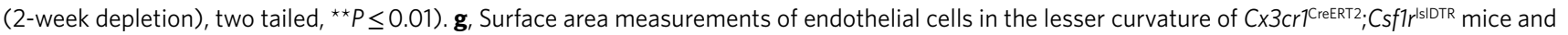
littermate controls ( $n=4$ mice per group, Mann-Whitney $t$-test, line at median; $P<0.0001$ (exact, 2-week depletion), ${ }^{\star \star \star \star} P \leq 0.0001$ ).

could predict that Mac ${ }^{\mathrm{AIR}}$ cells are particularly poised to be the progenitors of foam cells. This question was the focus of a recently published elegant study ${ }^{15}$. The authors found that, while Mac ${ }^{\mathrm{AIR}}$ cells can take up lipids, elimination of these cells only slightly delays but does not alter the burden of atherosclerotic lesions, nor does it change the accumulation of foam cells in lesions. Altogether, the conclusions of that study indicate that, while participating in the process, it is the influx of circulating monocytes into the neointima that is the main source of foam cells. In context, these data together with the fact that Mac ${ }^{\mathrm{AIR}}$ cells embed themselves in the tunica intima immediately after birth concur with the notion that the presence of these macrophages is not a response to a pathological insult.

The present report adds to the long list of studies that have recently identified self-renewing tissue-resident macrophages in multiple organs $\mathrm{s}^{21,22,28,51-54}$, now including the aortic endothelium in this list. These tissue-resident macrophages were found to seed multiple organs either during embryonic development or shortly thereafter and are derived from either yolk sac erythroid-myeloid or circulating myeloid progenitors ${ }^{27,28}$.

The findings presented here shift several paradigms. First, they challenge the concept that associations between the endothelium and leukocytes are always transient and triggered by acute inflammatory events. In fact, our results highlight a long-term partnership between endothelial cells and macrophages that is not dependent on immune responses; instead, it is triggered by the drastic hemodynamic changes associated with birth. Second, they change the view that a homotypic endothelial layer forms the luminal side of vessels, which now needs to be amended to include macrophages in areas of disturbed flow and aged arteries. Lastly, our findings indicate that intravascular clotting in arteries is constantly antagonized in regions of disturbed flow and the 'aged' endothelium. While the non-thrombogenic function of vessels was attributed exclusively to the endothelium, the data presented here provide clear evidence that, in some regions of the vascular tree, this can only be accomplished with the aid of macrophages.

\section{Methods}

Mice. All animal procedures were approved and performed in accordance with the University of California, Los Angeles (UCLA) and Northwestern University (NU) Institutional Animal Care and Use Committee. All other mouse information can be found in Supplementary Table 9. Mouse strains were maintained on a C57BL/6J background, with the exception of Csf1 $r^{\text {merCremer }}$ mice, which had a mixed background (FVB:C57BL/6). Mice were genotyped by Transnetyx. Male and female mice were used in approximately equal numbers for all experiments except for scRNA-seq experiments in which only male C57BL/6 mice were used to minimize potential sex and strain differences at the transcriptional level for the arch (8 weeks) and 'aged descending (78 weeks) A,B' datasets. However, validation of transcripts was performed in male and female mice. Unless specified, all adult mice used were $8-10$ weeks of age.

Aorta en face collection. Adult mice were injected intraperitoneally (i.p.) with $10 \mathrm{mg}$ methacholine to promote smooth muscle cell relaxation and facilitate en face imaging. Immediately after injection, mice were killed and perfused with $10 \mathrm{ml}$ $2 \%$ paraformaldehyde (PFA) through the left ventricle (for embryos and neonates, $0.5-3 \mathrm{ml} 2 \%$ PFA was used). Following perfusion, aortae were removed, and the adventitia was dissected under a microscope. Aortae were opened longitudinally, transferred to a $35-\mathrm{mm}$ silicon-coated dish filled with $2 \%$ PFA and pinned to lay flat, exposing the endothelium. Fixation proceeded for 1 additional hour at $4{ }^{\circ} \mathrm{C}$ followed by washes with PBS.

Aortic en face immunostaining. For immunostaining, tissue was washed three times with $1 \times$ Hank's balanced salt solution (HBSS) and incubated in blocking-permeabilization buffer ( $0.3 \%$ Triton X-100, 0.5\% Tween-20, 3\% normal donkey serum) for $1 \mathrm{~h}$. The primary antibody cocktail was prepared in blockingpermeabilization buffer and incubated overnight at $4{ }^{\circ} \mathrm{C}$ (the endothelial marker ERG, VE-Cad or PECAM1 were always used in conjunction with other markers to label the endothelium). Aortae were washed three times with $1 \times$ HBSS and incubated with secondary antibodies for $1 \mathrm{~h}$, washed with $1 \times$ HBSS and mounted on glass slides with ProLong Gold (Thermo, P36930). Antibodies and dilutions used are listed in Supplementary Table 9. Aortae were imaged using either an LSM880 confocal microscope (Zeiss) or an A1R HD25 confocal microscope (Nikon). $Z$-stack and tile scan features were used to image the large, wavy surfaces of the aortae. Resulting tiles were then stitched into a single large image (ZEN 2.0 Black software, Zeiss or NIS-Elements, Nikon), which enabled visualization of the large aortic arch at high resolution. Imaris software (Imaris 9.5.1 and 9.7.0, Bitplane) was used to visualize images in 3D. For a list of software used for analysis, see Supplementary Table 9. Additionally, Denoise.AI (Nikon) was employed to remove Poisson shot noise. Images were acquired using either $\times 20, \times 63$ or $\times 100$ objectives.

In vivo labeling. Mice were injected via the tail vein with either rat anti-mouse CD45 antibody or non-blocking PECAM1-specific (390)-daylight 650 antibody diluted in sterile PBS to label the lumen-facing surfaces of Mac ${ }^{\mathrm{AIR}}$ cells. Mice were killed $15 \mathrm{~min}$ after injection, and aortae were fixed, collected and stained with additional antibodies. For in vivo labeling of CD45, the following antibodies were additionally used: mouse anti-mouse CD45-biotin and streptavidin-A647, donkey anti-rat-A488 and anti-PECAM1 (2H8)-A568. For in vivo labeling of mice injected with PECAM1-specific (390) antibody, the following antibodies were used: anti-ERG and donkey anti-rabbit-A568, and anti-rat CD45 and donkey anti-ratA488 (Supplementary Table 9).

Immunostaining and imaging of sections. PFA-fixed, paraffin-embedded specimens from kidneys were sectioned at $4 \mu \mathrm{m}$. Antigen retrieval was performed using $1 \times$ citrate buffer, and then samples were incubated in blockingpermeabilization buffer for $1 \mathrm{~h}$. Sections were then incubated with primary antibodies (Supplementary Table 9) overnight at $4^{\circ} \mathrm{C}$. The following day, samples were incubated with species-specific secondary antibodies for $1 \mathrm{~h}$ before mounting in ProLong Gold. Samples were evaluated using an A1R HD25 confocal microscope (Nikon)

Bright-field imaging of aortae. Aortae from mice at E18.5, P1, P3, 1 week, 3 weeks and 10 weeks were dissected, and the adventitia was removed under a dissecting 
microscope. Aortae were pinned onto a 35-mm silicon-coated dish filled with PBS. Aortae were imaged using an Echo Revolve microscope (RVL-100-G).

Single-cell RNA sequencing. Isolation of intima cells was performed as previously described $^{20}$. In summary, mice were anesthetized and perfused with $10 \mathrm{ml}$ Versene buffer through the left ventricle. Under a dissecting microscope, the adventitia was removed, and the aorta was cut open in Versene buffer, exposing the endothelium. After Versene washes, aortae were bathed in $1 \times$ trypsin and incubated twice for $5 \mathrm{~min}$ at $37^{\circ} \mathrm{C}$. The endothelium was then gently removed using a microscalpel (EMS, 72046-30) and repeat pipetting, with sc-HBSS (containing 0.04\% BSA and $2 \%$ FBS to inactivate trypsin and actinomycin $\mathrm{D}$ at $1 \mu \mathrm{g} \mathrm{ml}^{-1}$ to block transcription applied to obtain a single-cell suspension (Extended Data Fig. 2a). Cells were pelleted at $300 \mathrm{~g}$ and then treated with $1 \times$ red blood cell (RBC) lysis buffer (eBioscience, 00-4333-57) for $1 \mathrm{~min}$ and washed twice with $0.04 \%$ BSA. To obtain enough cells, six male (78-week-old; 'aged descending') or eight male (8-week-old; arch) C57BL/6 mice were used per library.

To isolate adventitia cells into a single-cell suspension, mice were anesthetized and perfused with $10 \mathrm{ml}$ DMEM. The adventitia was dissected from aortae and dissociated using the Miltenyi Adipose Tissue Dissociation kit (130-105-808). Cells were pelleted at $300 \mathrm{~g}$. Following the Miltenyi protocol, the single-cell suspension was additionally treated with $1 \times$ RBC lysis buffer (eBioscience, 00-4333-57) and $1 \mathrm{U}$ DNase. The final cell suspension was washed multiple times and resuspended in $0.04 \%$ BSA.

scRNA-seq libraries were generated using the 10x Genomics Chromium Single Cell 3' Library \& Gel Bead kit version 2. Cells were loaded accordingly following the 10x Genomics protocol with an estimated targeted cell recovery of 5,000 cells. Sequencing was performed on the Illumina HiSeq 4000 (paired end, $100 \mathrm{bp}$ per read, 8-week-old mice, arch and 'aged descending A,B'). The digital expression matrix was generated by demultiplexing, processing barcodes and counting gene unique molecular indices using the Cellranger count pipeline (version 4.0.0, 10x Genomics). Multiple samples were merged using the Cellranger aggr pipeline. To identify different cell types and find signature genes for each cell type, the $\mathrm{R}$ package Seurat (version 3.1.2) was used. Cells that expressed $<100$ genes or $<500$ transcripts were filtered out. We ran the DoubletFinder algorithm and set the doublet rate at $2 \%$ as recommended by the vendor (10x Genomics). The algorithm predicted that only $0.01 \%$ of doublets were captured. Variable genes were selected using the FindVariableGenes function for further analysis. Data were normalized using the NormalizeData function with a scale factor of 10,000 . Genes were then scaled and centered using the ScaleData function. Principal-component analysis and $t$-SNE were used to reduce the dimensionality of the data. Cluster marker genes were found using the FindAllMarkers function. Cell types were annotated based on cluster marker genes. Heatmaps, violin plots and gene expression plots were generated by DoHeatmap, VlnPlot and featurePlot functions, respectively.

Flow cytometry of peripheral blood. To measure reporter labeling (lineage tracing) and donor chimerism (parabiotic mice) of circulating cells, blood was collected by retro-orbital bleeding into tubes containing FACS Buffer at $4{ }^{\circ} \mathrm{C}$. Blood cells were pelleted at $300 \mathrm{~g}$ and treated with $1 \times \mathrm{RBC}$ lysis buffer. Additionally, cells were stained on ice with anti-CD45-APC-Cy7 (BD, 557659) and then analyzed on a BD Fortessa machine.

Flow cytometry of organs. One day after DTx injection (Mac ${ }^{\text {AIR }}$ depletion), littermate control and $C x 3 \mathrm{Cr}^{\mathrm{CreERT2}}$; $C s f 1 r^{\text {slDTR }}$ mice were euthanized and perfused with $10 \mathrm{ml}$ Versene to remove blood and then perfused with $10 \mathrm{ml}$ DMEM. Lung, liver, kidney and leg bones (femur and tibia) were dissected and washed with PBS. For bone marrow collection, muscle was removed, and bones were cleaned, crushed in FACS Buffer using a motor pestle to release bone marrow cells and treated with $1 \times \mathrm{RBC}$ lysis buffer. For generation of single-cell suspensions from lung, liver and kidney, tissues were digested using Miltenyi's Lung Dissociation kit (130-095-927), Liver Dissociation kit (130-106-807) and multidissociation kit 2 (130-110-203), following the vendor's protocols. Cells were pelleted and treated with $1 \times \mathrm{RBC}$ lysis buffer. Additionally, bone marrow, lung, liver and kidney cells were stained on ice with anti-CD45-APC-Cy7 (BD, 557659), anti-CX3CR1-APC (BioLegend, 149008), anti-CSF1R-BV421 (BioLegend, 135513) and anti-TER119-PE-Cy7 (eBioscience, 25-5921-82) antibodies and then analyzed on a BD Fortessa machine.

Lineage tracing. Homozygous lox-stop-lox-tdTomato reporter mice were crossed with homozygous $C \times 3 \mathrm{cr} 1^{\text {CreERT2 }}$ mice. Tamoxifen induction of Cre activity in the resulting $\mathrm{F}_{1}$ compound heterozygotes was initiated by dissolving tamoxifen (free base, MP Biomedicals, 0215673891) in sunflower seed oil (Sigma, S5007) and administering $0.01 \mathrm{mg}$ tamoxifen via oral gavage to neonates at P1, P3 and P5. The penetration of intimal tdTomato ${ }^{+} \mathrm{CD} 45^{+}$cells in the aorta at $\mathrm{P} 7$ ranged from $40 \%$ to $62 \%$, determined by en face confocal imaging (Fig. 3f); thus, analysis of 4 -week-old adult mice needed to be normalized to data from littermates at P7. For baseline controls, half of the litter was killed, and blood as well as aortae were collected to determine reporter labeling efficiency. At 4 weeks, the remaining littermates were killed, and blood as well as aortae were collected to determine reporter expression.

Reporter expression in adult $C x 3 c r 1^{\text {CreERT2}} ; R 26^{\text {lox-stop-lox-tdTomato }}$ mice was induced after i.p. injection of $1 \mathrm{mg}$ tamoxifen three times, every other day. The penetration of tdTomato-positive intimal CD $45^{+}$cells in the aorta ranged from $97 \%$ to $100 \%$. Aortae and blood were collected at 1 week after tamoxifen treatment as a baseline control for normalization purposes. Littermates were killed 10 weeks after injection to assess retention of reporter expression in adulthood.

For clonal tracing, $C \times 3 \mathrm{cr} 1^{\text {CreERT2 }} ; R 26^{\text {Rainbow }}\left(\right.$ ref. ${ }^{55}$ ) neonates were administered $0.01 \mathrm{mg}$ tamoxifen via oral gavage at P1, P3 and P5 to yield permanent expression of three mutually exclusive fluorescent proteins: Cerulean, mOrange and mCherry. At 8 weeks, mice were killed, and reporter labeling was assessed. Adult $C \times 3 c r 1^{\text {CreERT }}$ ${ }^{2} ; R 26^{\text {Rainbow }}$ mice were injected with one dose of $0.02 \mathrm{mg}$ tamoxifen, and aortae were assessed 9 months later (at 11 months of age)

In vivo macrophage depletion. To deplete macrophages, adult (8-10-week-old) $\mathrm{C} \times 3 \mathrm{cr} 1^{\text {CreERT2}} ;$ Csf $1 r^{\text {sIDTR }}$ mice were first injected with tamoxifen i.p. to promote Cre-dependent induction of the DTR. The following day, mice were injected with $200 \mathrm{ng}$ DTx (Sigma, D0564) in sterile $1 \times$ PBS to induce apoptosis of DTR-expressing cells. This resulted in cell death of this population in as quickly as $24 \mathrm{~h}$. However, as intimal, aortic myeloid cells are replenished quickly, evaluation of longer time points required continuous injection with tamoxifen followed by DTx every $3 \mathrm{~d}$ until the end of the experiment.

EdU-incorporation assay. Mice were injected i.p. with EdU (Fisher Scientific, A10044 at $10 \mathrm{mM})$. Two hours after injection, mice were terminally anesthetized and perfused with $2 \%$ PFA. The aorta was removed, and the adventitia was dissected. Aortae were longitudinally cut to exposed the endothelium. Following fixation, EdU was revealed using A647 following the manufacture's protocol (Invitrogen, C10640). Additionally, aortae were stained for the nuclear endothelial marker ERG and the pan-hematopoietic marker CD45 and with DAPI before being imaged (Supplementary Table 9).

Scanning election microscopy. Mice were anesthetized and perfused with $10 \mathrm{ml} 2 \%$ PFA through the left ventricle. Aortae were first immunostained en face following protocols listed above. High- and low-resolution images were then obtained by confocal microscopy. Aortae were then washed with $1 \times$ HBSS, dehydrated with increasing concentrations of ethanol and subjected to critical point drying followed by gold and/or palladium coating using a sputter coater. High- and low-resolution SEM images were also taken to use landmarks and find macrophages identified with the confocal images. Confocal and SEM images were then overlaid together using Adobe Photoshop (22.5.0).

To image microclots in aortae from macrophage-depleted $\left(\mathrm{C} x 3 \mathrm{cr} 1^{\mathrm{CreERT} 2}\right.$; Csfl $\left.1 r^{\text {sIDTR }}\right)$ and control mice, mice were perfused with $4 \%$ glutaraldehyde through the left ventricle. Aortae were dissected and treated for an additional hour with $4 \%$ glutaraldehyde at room temperature and then washed several times with $1 \times$ PBS. Aortae were incubated in $1 \%$ osmium tetroxide for $1 \mathrm{~h}$, dehydrated with a series of ethanol, dried to the critical point, mounted on pins and coated with 10-nm gold particles for SEM. Aortae were then imaged using a JEOL NeoScope microscope at $10 \mathrm{kV}$ or $15 \mathrm{kV}$.

Dabigatran treatment. Mice were injected twice daily (morning and night) i.p. with $300 \mu$ g dabigatran (BIBR 953, Selleck, S2196) per injection for $5 \mathrm{~d}$. Dosage was determined by the clotting test. After $5 \mathrm{~d}$ of treatment, mice were sacrificed, and aortae were analyzed.

In vivo siFibrinogen knockdown. Mice were injected with either siFibrinogen or an siRNA targeting luciferase (siLuciferase, control) at $1 \mathrm{mg}$ siRNA per $\mathrm{kg}$ body weight via tail vein injection. siFibrinogen and siLuciferase were each encapsulated in lipid nanoparticles, composed of an ionizable lipid (DLin-MC3-DMA), phosphatidylcholine, cholesterol and a polyethylene glycol lipid, using methods previously described ${ }^{56}$. To determine knockdown efficiency, we quantified fibrinogen protein levels in plasma $7 \mathrm{~d}$ after injection. For this, we collected peripheral blood in EDTA-coated tubes (BD, 365974) and isolated plasma by centrifugation. Plasma was diluted (1:50), and fibrinogen levels were measured using a Mouse Fibrinogen ELISA kit (Abcam, ab213478).

Intravenous administration of fbg-a488 or plasminogen-FITC. Mice were injected intravenously with $400 \mu \mathrm{g}$ fbg-a 488 (Thermo, F13191), plasminogenFITC (Abcam, ab92770) or albumin-FITC (control, Thermo, A23015). Mice injected with fbg-a 488 were killed $3 \mathrm{~h}$ after injection, and mice injected with plasminogen-FITC were harvested $4 \mathrm{~h}$ after injection to assess local accumulation relative to the $\mathrm{Mac}^{\mathrm{AIR}}$ cells.

D-dimer measurements. Mice were anesthetized, and blood was collected via right ventricle puncture with a $25 \mathrm{G}$ syringe. For D-dimer measurements, blood was collected in citrate buffer with a final concentration of $3.2 \%$ citrate (for D-dimer). Blood was centrifuged, and citrate plasma was collected and shipped to IDEXX on dry ice for quantification.

Comparison to the Tabula Muris Atlas and the Chakarov et al. study. Mac ${ }^{\mathrm{AIR}}$ cells were compared to monocytes or macrophages from the Tabula Muris Atlas ${ }^{25}$. Specifically, expression values for all monocytes and macrophages were extracted 
from the Tabula Muris Atlas and merged with expression values of Mac ${ }^{\mathrm{AIR}}$ cells from our dataset. The Seurat pipeline described above was applied to cluster cells. As the datasets were generated by different laboratories, the RunHarmony function from the R package Harmony ${ }^{57}$ was applied to remove potential batch effects among different tissues. After clustering, Mac ${ }^{\mathrm{AIR}}$ marker genes were used to calculate a module score, which was used to identify the cell population from the Tabula Muris Atlas that was similar to $\mathrm{Mac}^{\mathrm{AIR}}$ cells. The module score was calculated based on the average expression of genes in the list, subtracted by the aggregated expression of randomly chosen control genes.

Our monocyte and macrophage scRNA-seq datasets were also overlapped with data from the two macrophage populations obtained by Chakarov et al. ${ }^{23}$. For this marker, genes extracted from the comparison between monocytes, adventitial macrophages and $\mathrm{Mac}^{\mathrm{AIR}}$ cells in our study were compared to data from Lyve $1^{\mathrm{lo}} M H C I I^{\mathrm{hi}}$ and $L y v e 1^{\mathrm{hi}} M H C I I^{\mathrm{lo}}$ cells (MHCII complex contains multiple genes: M2-Aa, M2-Ab1, M2-Eb2, M2-Eb1) from the Chakarov et al. study. The Jaccard index between each pair for cell types from the two datasets was calculated and plotted in the heatmap.

Parabiosis. Mice of similar weight and sex were housed together for 2 weeks before surgery to assess compatibility. Surgeries were performed as described previously ${ }^{58}$. In short, mice were anesthetized, and matching skin incisions were made from the olecranon to the knee joint of each mouse, and the subcutaneous fascia was bluntly dissected to create $\sim 0.5 \mathrm{~cm}$ of free skin. The right olecranon of one animal was attached to the left olecranon of the other by a single 3-0 nylon suture and tie. The partners' knee joints were similarly connected. Dorsal and ventral skins were closed, approximated by staples, and the animals were warmed with heating pads and monitored until recovery. Parabiotic pairs were housed one pair per cage and given acidified water ( $\mathrm{pH}$ 2.5). After 4 weeks of anastomosis, blood samples from each animal in a parabiont pair were analyzed using flow cytometry. Animal pairs with $<30 \%$ blood chimerism were excluded from our studies.

Annexin staining in aortae. Adult mice were killed and perfused with $3 \mathrm{ml} 5 \%$ Annexin-A488 conjugate (Invitrogen, A13201) through the left ventricle. Five minutes later, the aorta was dissected and transferred to a $35-\mathrm{mm}$ silicon-coated dish filled with 20\% Annexin-A488. Annexin-A488 (20\%) was also used to flush the Annexin inside the intact vessel and then incubated at $37^{\circ} \mathrm{C}$ for 10 min. Following incubation, tissue was fixed in 2\% PFA for 1 additional hour at room temperature, followed by multiple washes with PBS. Aortae were then opened and pinned to lay flat, exposing the endothelium and immunostained as described previously with anti-ERG and anti-CD45 antibodies and DAPI (Supplementary Table 9).

In vitro thrombin and MMP13 treatment and thrombin treatment with shear stress. HAECs were isolated as described from aortic trimmings of donor hearts at UCLA $^{59}$. Cells were de-identified and exempt from consideration as human individuals by institutional regulatory boards at the UCLA. Additional HAECs were purchased from Lonza (CC-2535). HAECs were grown on gelatin-coated, tissue culture-treated six-well plates (Falcon, 353046) to confluency in complete MCDB-131 medium (Vec Technologies, MCDB131-WOFBS) with 10\% FBS (Omega Scientific, FB-11). Cells were cultured in a humidified incubator at $37^{\circ} \mathrm{C}$ with $5 \% \mathrm{CO}_{2}$ and used between passages 4 and 9. Once confluent, cells were washed with PBS to remove serum and incubated with serum-free MCDB-131 medium. The next day, cells were treated with vehicle, MMP13 ( $1 \mu \mathrm{g}, \mathrm{Abcam}$, ab227435) or thrombin $\left(0.625 \mathrm{U} \mathrm{ml}^{-1}\right.$, Sigma, 10602400001) for $5 \mathrm{~min}$. After treatment, cells were washed with phosphate buffered saline (PBS) containing $\mathrm{Na}_{3} \mathrm{OV}_{4}$, and lysates were collected using mRIPA (50 mM Tris, $0.1 \%$ SDS, $0.5 \%$ sodium deoxycholate, $1 \%$ Triton $\mathrm{X}-100,150 \mathrm{mM} \mathrm{NaCl}, 1 \mathrm{mM} \mathrm{Na}_{3} \mathrm{VO}_{4}$, protease inhibitor cocktail). Lysates were denatured in Laemmli buffer with 2-mercaptoethanol at $95^{\circ} \mathrm{C}$ for $10 \mathrm{~min}$. Proteins were separated with an SDSPAGE gradient (4-20\%, Bio-Rad, 4561094DC) gel, transferred onto nitrocellulose membranes (Bio-Rad, 1704271) and incubated overnight at $4{ }^{\circ} \mathrm{C}$ with primary antibodies. Primary antibodies were anti-pMLC2 (3671S), anti-MLC2 (CST, 3672S), anti-PAR1 (Thermo, PA5-19102) and anti- $\gamma$-tubulin (Abcam, ab11321). HRP-conjugated secondary antibodies were applied in a species-dependent manner for $1 \mathrm{~h}$. Immunocomplex detection was performed with the enhanced chemiluminescence SuperSignal West Femto Maximum Sensitivity Substrate (Thermo Fisher Scientific, 34095) using the ChemiDoc XRS+ Molecular Imager (Bio-Rad Laboratories). Quantification of bands was performed using Image Lab Software (Bio-Rad Laboratories).

For thrombin and shear stress experiments, HAECs were grown on gelatin-coated, glass-bottom, six-well plates (Cellvis, P06-1.5H-N) to confluency in complete MCDB-131 medium supplemented with $10 \%$ FBS. Once confluent, cells were washed with PBS to remove serum, and medium containing MCDB-131 and $4 \%$ dextran (Sigma-Aldrich, 31392) was added to confluent HAECs. Vehicle (PBS) or thrombin (Sigma, 10602400001) at a final concentration of $0.625 \mathrm{U} \mathrm{ml}^{-1}$ was then applied. Monolayers were subjected to unidirectional constant laminar flow for $48 \mathrm{~h}$ at 130 r.p.m. in a horizontal circular orbit (Benchmark, BT302). Static monolayers used the same dextran-containing medium and were cultured alongside flow-treated monolayers. After flow treatment $(48 \mathrm{~h})$, cells were fixed in 2\% PFA for $15 \mathrm{~min}$ at room temperature and then washed with PBS.

For immunostaining, cells were incubated in blocking-permeabilization buffer $(0.3 \%$ Triton X-100, 0.5\% Tween-20, 3\% normal donkey serum) for $1 \mathrm{~h}$ at room temperature. The primary antibody cocktail was prepared in blockingpermeabilization buffer and incubated with samples overnight at $4{ }^{\circ} \mathrm{C}$. The following day, aortae were washed three times with $1 \times$ PBS and incubated with secondary antibodies for $1 \mathrm{~h}$ at room temperature.

Microsphere permeability. One day after DTx injection (Mac ${ }^{\text {AIR }}$ depletion), littermate control and $C x 3 \mathrm{crl}^{\mathrm{CreERT2}} ; \mathrm{Csf1} 1 r^{\mathrm{ISITR}}$ mice were injected in the left ventricle with $200 \mu \mathrm{l} 40$-nm microspheres (1:10, Thermo, F8795). Beads circulated for $5 \mathrm{~min}$, and then mice were euthanized and perfused with $10 \mathrm{ml}$ PBS, followed by $10 \mathrm{ml} 2 \%$ PFA. Aortae were collected, and whole-mount staining was performed as described above. For the positive control, C57BL/6 mice were injected with $50 \mathrm{mM}$ EDTA buffer solution in the left ventricle, which was allowed to circulate for $5 \mathrm{~min}$ to challenge endothelial junctions. Next, these positive control mice were injected with microspheres and harvested as described.

Tail bleeding. One day after DTx injection (Mac ${ }^{\mathrm{AIR}}$ depletion), littermate control and $C \times 3 \mathrm{crl}^{\mathrm{CreERT2}} ; \mathrm{Cs} f 1 r^{\text {slDTR }}$ mice with same age and weight were anesthetized with ketamine-xylazine at a dose of $0.1 \mathrm{ml}$ per $20 \mathrm{~g}$. Using scalpel blade \#11, tails of mice were resected exactly $3 \mathrm{~mm}$ distal from the tail end. Tails were quickly placed in tail-bleeding buffer ( $10 \mathrm{mM}$ Tris- $\mathrm{HCl}, 2 \mathrm{mM} \mathrm{CaCl}_{2}$, warmed to $37^{\circ} \mathrm{C}$ ), and a stopwatch was started. The stopwatch was stopped after the blood stream halted.

Ex vivo nitric oxide measurements. A $5 \mathrm{mM}$ stock solution of 4 -amino5-methylamino-2 ${ }^{\prime}, 7^{\prime}$-difluorofluorescein (DAF-FM) diacetate (Invitrogen, D3844) was diluted to $10 \mu \mathrm{M}$ in medium without phenol red (EBM, Lonza, CC-3129). One day after DTx injection (Mac ${ }^{\text {AIR }}$ depletion), littermate control and $\mathrm{C} x 3 \mathrm{cr} 1^{\mathrm{CreERT} 2}$; Csf1 $r^{\text {sIDTR }}$ mice were euthanized and perfused with $10 \mathrm{ml}$ PBS followed by $3 \mathrm{ml}$ $10 \mu \mathrm{M}$ DAF-FM diacetate. Aortae were then quickly and gently collected in $10 \mu \mathrm{M}$ DAF-FM. Intact aortae were then incubated in DAF-FM for $10 \mathrm{~min}$ at $37^{\circ} \mathrm{C}$. After $10 \mathrm{~min}, \mathrm{DAF}-\mathrm{FM}$ was replaced with fresh DAF-FM, and the sample was incubated for an additional $15 \mathrm{~min}$. Aortae were then quickly filleted open and mounted with PBS. Confocal imaging occurred immediately after to evaluate nitric oxide levels.

Quantification and statistical analysis. Treatments were randomized; investigators were blinded to allocation for outcome assessment. No samples or animals were excluded from this study. Sampling size for animal experiments was determined by power analysis with a type 1 error rate of $5 \%$ and a minimum detectable effect of $20 \%$. For cell surface experiments, we used sampling similar to previous published reports. For experiments for which the outcome was immunofluorescence, figures show representative images; however, the number of independent times that the experiment was reproduced using biological replicates is provided in the legend.

Quantification of intimal immune cells in aortae was performed using the spots function in Imaris 9.5.1 or 9.7.0 (Bitplane) on maximum-intensity $Z$ projections. Only cells with clearly distinguishable bodies and nuclei (DAPI, not shown) were quantified. No samples or animals were excluded from this study.

Cell surface area and elongation factors of endothelial cells in aortae of control and macrophage-depleted $\left(C \times 3 \mathrm{cr}^{\mathrm{CreERT2}} ; C s f 1 r^{\text {ISIDTR }}\right)$ mice was determined in several regions of $44.1 \mathrm{~mm}^{2}$ within the lesser curvature of each whole-mount, flat-mounted aorta. Measurements were performed with NIS-Elements, using a combination of manual and automated cell shape identification. VE-Cad or PECAM1 staining was used to define endothelial cell borders. For macrophage-depletion experiments, we used littermate controls that received both tamoxifen and DTx injections. For evaluation of fluorescence, mean fluoresce intensity was determined using the surface function in Imaris 9.5.1 or 9.7.0 on maximum-intensity $Z$ projections. Subsequently, the mean for at least three independent samples and s.d. was determined. To assess whether two datasets were significantly different, we calculated $P$ values with unpaired, nonparametric Student's $t$-test followed by the Mann-Whitney test; $P<0.05$ was considered significant $\left({ }^{\star} P<0.05,{ }^{* \star} P<0.01\right.$, $\left.{ }^{* * *} P<0.001,{ }^{* * * *} P<0.0001\right)$. Statistical analyses were performed with Prism 8 (GraphPad Software).

Reporting Summary. Further information on research design is available in the Nature Research Reporting Summary linked to this article.

\section{Data availability}

All data generated or analyzed are included in the main article and associated files. scRNA-seq data were deposited in the GEO database under accession numbers GSE161787, GSE125691 (ref. ${ }^{22}$ ) and GSE154817 (ref. ${ }^{15}$ ). The Tabula Muris Atlas dataset is deposited at https://www.czbiohub.org/tabula-muris $/ 25$. Source data are provided with this paper.

Received: 13 June 2021; Accepted: 17 November 2021; Published online: 12 January 2022 


\section{References}

1. Diacovo, T. G., Roth, S. J., Buccola, J. M., Bainton, D. F. \& Springer, T. A. Neutrophil rolling, arrest, and transmigration across activated, surface-adherent platelets via sequential action of P-selectin and the $\beta_{2}$-integrin CD11b/CD18. Blood 88, 146-157 (1996).

2. Ley, K., Laudanna, C., Cybulsky, M. I. \& Nourshargh, S. Getting to the site of inflammation: the leukocyte adhesion cascade updated. Nat. Rev. Immunol. 7, 678-689 (2007).

3. Liew, P. X. \& Kubes, P. The neutrophil's role during health and disease. Physiol. Rev. 99, 1223-1248 (2019).

4. Mamdouh, Z., Chen, X., Pierini, L. M., Maxfield, F. R. \& Muller, W. A. Targeted recycling of PECAM from endothelial surface-connected compartments during diapedesis. Nature 421, 748-753 (2003).

5. Gisterå, A. \& Hansson, G. K. The immunology of atherosclerosis. Nat. Rev. Nephrol. 13, 368-380 (2017).

6. Quintar, A. et al. Endothelial protective monocyte patrolling in large arteries intensified by western diet and atherosclerosis. Circ. Res. 120, 1789-1799 (2017)

7. Libby, P., Nahrendorf, M. \& Swirski, F. K. Leukocytes link local and systemic inflammation in ischemic cardiovascular disease: an expanded "cardiovascular continuum". J. Am. Coll. Cardiol. 67, 1091-1103 (2016).

8. Robbins, C. S. et al. Local proliferation dominates lesional macrophage accumulation in atherosclerosis. Nat. Med. 19, 1166-1172 (2013).

9. Ross, R. Rous-Whipple Award Lecture. Atherosclerosis: a defense mechanism gone awry. Am. J. Pathol. 143, 987-1002 (1993).

10. Auffray, C. et al. Monitoring of blood vessels and tissues by a population of monocytes with patrolling behavior. Science 317, 666-670 (2007)

11. Carlin, L. M. et al. Nr4a1-dependent Ly6C $\mathrm{C}^{\text {low }}$ monocytes monitor endothelial cells and orchestrate their disposal. Cell 153, 362-375 (2013).

12. Hanna, R. N. et al. Patrolling monocytes control tumor metastasis to the lung. Science 350, 985-990 (2015).

13. Choi, J.-H. et al. Identification of antigen-presenting dendritic cells in mouse aorta and cardiac valves. J. Exp. Med. 206, 497-505 (2009).

14. Jongstra-Bilen, J. et al. Low-grade chronic inflammation in regions of the normal mouse arterial intima predisposed to atherosclerosis. J. Exp. Med. 203, 2073-2083 (2006).

15. Williams, J. W. et al. Limited proliferation capacity of aortic intima resident macrophages requires monocyte recruitment for atherosclerotic plaque progression. Nat. Immunol. 21, 1194-1204 (2020).

16. Broadhouse, K. M. et al. Assessment of PDA shunt and systemic blood flow in newborns using cardiac MRI. NMR Biomed. 26, 1135-1141 (2013).

17. Colucci-Guyon, E. et al. Mice lacking vimentin develop and reproduce without an obvious phenotype. Cell 79, 679-694 (1994).

18. Salvador, J. et al. Transcriptional evaluation of the ductus arteriosus at the single-cell level uncovers a requirement for vimentin for complete closure. Preprint at bioRxiv https://doi.org/10.1101/2021.10.30.466605 (2021).

19. Ma, F., Hernandez, G. E., Romay, M. \& Iruela-Arispe, M. L. Single-cell RNA sequencing to study vascular diversity and function. Curr. Opin. Hematol. 28, 221-229 (2021).

20. McDonald, A. I. et al. Endothelial regeneration of large vessels is a biphasic process driven by local cells with distinct proliferative capacities. Cell Stem Cell 23, 210-225 (2018).

21. Ensan, S. et al. Self-renewing resident arterial macrophages arise from embryonic CX3CR1 ${ }^{+}$precursors and circulating monocytes immediately after birth. Nat. Immunol. 17, 159-168 (2016).

22. Chakarov, S. et al. Two distinct interstitial macrophage populations coexist across tissues in specific subtissular niches. Science 363, eaau0964 (2019).

23. Lim, H. Y. et al. Hyaluronan receptor LYVE-1-expressing macrophages maintain arterial tone through hyaluronan-mediated regulation of smooth muscle cell collagen. Immunity 49, 326-347 (2018)

24. Hernandez, G. E. \& Iruela-Arispe, M. L. The many flavors of monocyte/ macrophage-endothelial cell interactions. Curr. Opin. Hematol. 27, 181-189 (2020).

25. Tabula Muris Consortium et al. Single-cell transcriptomics of 20 mouse organs creates a Tabula Muris. Nature 562, 367-372 (2018).

26. Satpathy, A. T. et al. Zbtb46 expression distinguishes classical dendritic cells and their committed progenitors from other immune lineages. J. Exp. Med. 209, 1135-1152 (2012).

27. Epelman, S., Lavine, K. J. \& Randolph, G. J. Origin and functions of tissue macrophages. Immunity 41, 21-35 (2014).

28. Ginhoux, F. \& Guilliams, M. Tissue-resident macrophage ontogeny and homeostasis. Immunity 44, 439-449 (2016).

29. Serbina, N. V. \& Pamer, E. G. Monocyte emigration from bone marrow during bacterial infection requires signals mediated by chemokine receptor CCR2. Nat. Immunol. 7, 311-317 (2006).

30. Boyer, S. W., Schroeder, A. V., Smith-Berdan, S. \& Forsberg, E. C. All hematopoietic cells develop from hematopoietic stem cells through Flk2/ Flt3-positive progenitor cells. Cell Stem Cell 9, 64-73 (2011).
31. Epelman, S. et al. Embryonic and adult-derived resident cardiac macrophages are maintained through distinct mechanisms at steady state and during inflammation. Immunity 40, 91-104 (2014).

32. Mehta, D. \& Malik, A. B. Signaling mechanisms regulating endothelial permeability. Physiol. Rev. 86, 279-367 (2006).

33. Rigor, R. R., Shen, Q., Pivetti, C. D., Wu, M. H. \& Yuan, S. Y. Myosin light chain kinase signaling in endothelial barrier dysfunction. Med. Res. Rev. 33, 911-933 (2013).

34. Austin, K. M., Covic, L. \& Kuliopulos, A. Matrix metalloproteases and PAR1 activation. Blood 121, 431-439 (2013).

35. Hou, H.-H. et al. MMP-12 activates protease-activated receptor-1, upregulates placenta growth factor, and leads to pulmonary emphysema. Am. J. Physiol. Lung Cell. Mol. Physiol. 315, L432-L442 (2018).

36. Jaffré, F., Friedman, A. E., Hu, Z., Mackman, N. \& Blaxall, B. C. $\beta$-adrenergic receptor stimulation transactivates protease-activated receptor 1 via matrix metalloproteinase 13 in cardiac cells. Circulation 125, 2993-3003 (2012).

37. Willis Fox, O. \& Preston, R. J. S. Molecular basis of protease-activated receptor 1 signaling diversity. J. Thromb. Haemost. 18, 6-16 (2020).

38. Kulkarni, J. A., Witzigmann, D., Chen, S., Cullis, P. R. \& van der Meel, R. Lipid nanoparticle technology for clinical translation of siRNA therapeutics. Acc. Chem. Res. 52, 2435-2444 (2019).

39. Bugge, T. H. et al. Loss of fibrinogen rescues mice from the pleiotropic effects of plasminogen deficiency. Cell 87, 709-719 (1996).

40. Prasad, J. M. et al. Mice expressing a mutant form of fibrinogen that cannot support fibrin formation exhibit compromised antimicrobial host defense. Blood 126, 2047-2058 (2015).

41. Motley, M. P. et al. A CCR2 macrophage endocytic pathway mediates extravascular fibrin clearance in vivo. Blood 127, 1085-1096 (2016).

42. Bonnardel, J. et al. Stellate cells, hepatocytes, and endothelial cells imprint the Kupffer cell identity on monocytes colonizing the liver macrophage niche. Immunity 51, 638-654 (2019).

43. Sakai, M. et al. Liver-derived signals sequentially reprogram myeloid enhancers to initiate and maintain Kupffer cell identity. Immunity $\mathbf{5 1}$ 655-670 (2019).

44. Scott, C. L. et al. Bone marrow-derived monocytes give rise to self-renewing and fully differentiated Kupffer cells. Nat. Commun. 7, 10321 (2016).

45. Colvin, R. B. \& Dvorak, H. F. Fibrinogen/fibrin on the surface of macrophages: detection, distribution, binding requirements, and possible role in macrophage adherence phenomena. J. Exp. Med. 142, 1377-1390 (1975).

46. Hiller, O., Lichte, A., Oberpichler, A., Kocourek, A. \& Tschesche, H. Matrix metalloproteinases collagenase-2, macrophage elastase, collagenase- 3 , and membrane type 1-matrix metalloproteinase impair clotting by degradation of fibrinogen and factor XII. J. Biol. Chem. 275, 33008-33013 (2000).

47. Motterle, A. et al. Influence of matrix metalloproteinase-12 on fibrinogen level. Atherosclerosis 220, 351-354 (2012).

48. Tardif, G., Reboul, P., Pelletier, J.-P. \& Martel-Pelletier, J. Ten years in the life of an enzyme: the story of the human MMP-13 (collagenase-3). Mod. Rheumatol. 14, 197-204 (2004).

49. Bugge, T. H., Flick, M. J., Daugherty, C. C. \& Degen, J. L. Plasminogen deficiency causes severe thrombosis but is compatible with development and reproduction. Genes Dev. 9, 794-807 (1995).

50. Goguen, J. D., Bugge, T. \& Degen, J. L. Role of the pleiotropic effects of plasminogen deficiency in infection experiments with plasminogen-deficient mice. Methods 21, 179-183 (2000)

51. Gomez Perdiguero, E. et al. Tissue-resident macrophages originate from yolk-sac-derived erythro-myeloid progenitors. Nature $\mathbf{5 1 8}$, 547-551 (2015)

52. Hoeffel, G. et al. C-Myb ${ }^{+}$erythro-myeloid progenitor-derived fetal monocytes give rise to adult tissue-resident macrophages. Immunity 42, 665-678 (2015)

53. Schulz, C. et al. A lineage of myeloid cells independent of Myb and hematopoietic stem cells. Science 336, 86-90 (2012).

54. Yona, S. et al. Fate mapping reveals origins and dynamics of monocytes and tissue macrophages under homeostasis. Immunity 38, 79-91 (2013).

55. Rinkevich, Y., Lindau, P., Ueno, H., Longaker, M. T. \& Weissman, I. L. Germ-layer and lineage-restricted stem/progenitors regenerate the mouse digit tip. Nature 476, 409-413 (2011).

56. Strilchuk, A. W. et al. Sustained depletion of FXIII-A by inducing acquired FXIII-B deficiency. Blood 136, 2946-2954 (2020).

57. Korsunsky, I. et al. Fast, sensitive and accurate integration of single-cell data with Harmony. Nat. Methods 16, 1289-1296 (2019).

58. Ali, S. R. et al. Developmental heterogeneity of cardiac fibroblasts does not predict pathological proliferation and activation. Circ. Res. 115, 625-635 (2014)

59. Navab, M. et al. Monocyte migration into the subendothelial space of a coculture of adult human aortic endothelial and smooth muscle cells. J. Clin. Invest. 82, 1853-1863 (1988). 


\section{Acknowledgements}

We are grateful to G. Randolph and J. Williams for extensive discussions about intima resident macrophages and for the generous transfer of the $C x 3 \mathrm{cr} 1^{\text {CreERT }}$ Csf $1 r^{\text {sIDTR }}$ transgenic mouse. We are also thankful to W. Mueller for anti-PECAM1 antibodies. We are thankful to K. Ridge for the generous transfer of $\mathrm{Vim}^{-1-}$ mice. We also thank the Broad Stem Cell Research Center and the Jonsson Comprehensive Center at the UCLA for sequencing scRNA-seq libraries; the Broad Stem Cell Research Center flow core at the UCLA and the Robert H. Lurie Comprehensive Cancer Center flow core at NU, Feinberg School of Medicine; the Mouse Histology and Phenotyping core at NU; and the Center for Advanced Microscopy at NU. Special thanks to M. Steel and S. Mirkov for managing animal colonies and for assistance with husbandry and animal experiments. Additionally, we thank S. Buchanan for help with cell size quantification. We also thank F.V. Korobova from the Center for Advanced Microscopy at NU for support with SEM. We thank members of the Arispe laboratory and the Feinberg Cardiovascular and Renal Research Institute at NU for extensive discussions. Illustrations were created by C. Bauista or with https://biorender.com/. This work was supported by R35HL140014 to M.L.I.-A. and a Howard Hughes Medical Institute Gilliam Fellowship (GT11560) to G.E.H. J.S. is supported by the NU Molecular and Translational Cardiovascular Training Program (T32HL134633, SP0040691). D.A.L. is supported by the NIH-NICHD training grant T32HD007491.

\section{Author contributions}

G.E.H. performed and designed experiments and wrote and edited the manuscript; G.M., N.B.F., J.S. and D.A.L. performed experiments and quantification; L.J.J., J.L. and C.J.K. provided siRNA-nanoparticles; P.Z. performed parabiosis experiments, supervised by R.A. R.A. provided transgenic mice and provided intellectual input and discussion; A.E.B. and M.J.F. provided key transgenic mice and provided intellectual input for experimental design and analysis. M.P. provided intellectual input for experiments using scRNA-seq data; F.M. performed bioinformatic analysis of scRNA-seq data; M.L.I.-A. conceived the study, designed experiments and wrote and edited the manuscript. All authors discussed the results and had the opportunity to comment on the manuscript.

\section{Competing interests}

C.J.K. is a director and shareholder of NanoVation Therapeutics Inc., which is commercializing RNA-based therapies. C.J.K., L.J.J. and J.L. have filed intellectual property on RNA-based therapies, with the intention of commercializing these inventions. All other authors declare no competing interests.

\section{Additional information}

Supplementary information The online version contains supplementary material available at https://doi.org/10.1038/s44161-021-00006-4.

Correspondence and requests for materials should be addressed to M. Luisa Iruela-Arispe.

Peer review information Nature Cardiovascular Research thanks Klaus Ley, John Hwa and the other, anonymous, reviewer(s) for their contribution to the peer review of this work.

Reprints and permissions information is available at www.nature.com/reprints.

Publisher's note Springer Nature remains neutral with regard to jurisdictional claims in published maps and institutional affiliations.

\section{(c) (i)}

Open Access This article is licensed under a Creative Commons

Attribution 4.0 International License, which permits use, sharing, adaptation, distribution and reproduction in any medium or format, as long as you give appropriate credit to the original author(s) and the source, provide a link to the Creative Commons license, and indicate if changes were made. The images or other third party material in this article are included in the article's Creative Commons license, unless indicated otherwise in a credit line to the material. If material is not included in the article's Creative Commons license and your intended use is not permitted by statutory regulation or exceeds the permitted use, you will need to obtain permission directly from the copyright holder. To view a copy of this license, visit http://creativecommons. org/licenses/by/4.0/.

(c) The Author(s) 2022 

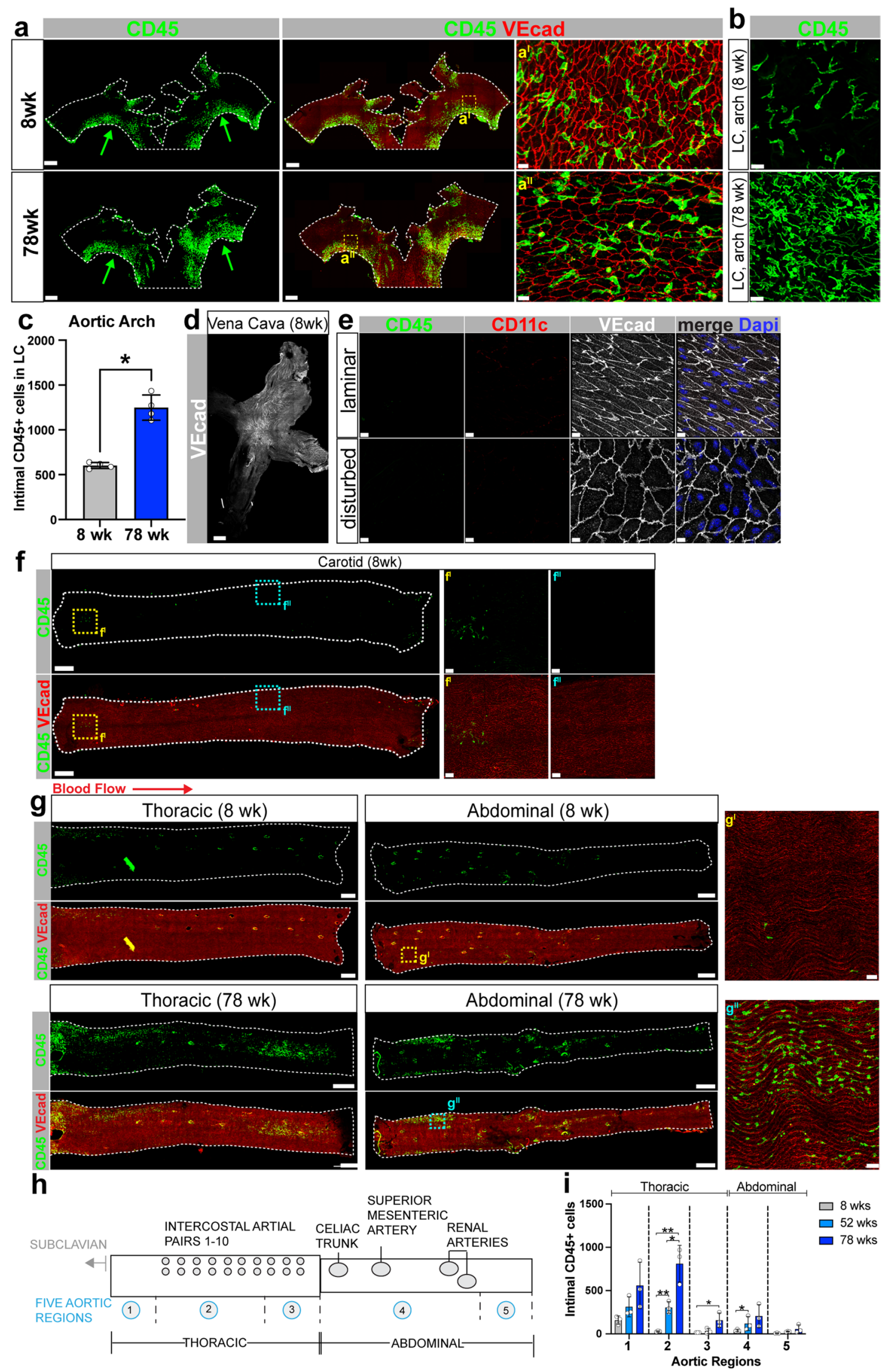

Extended Data Fig. 1 | See next page for caption. 
Extended Data Fig. 1 | Intimal immune cell distribution in the aorta and vena cava of young and aged mice. a, Accumulation of intimal immune cells, as per CD45 (green arrows) in adult $\left(n=498\right.$ mice) and old $78 w k\left(n=6\right.$ mice) aortic arch. VE-Cadherin (red). Scale bars, 500 $\mu m \& 15 \mu m\left(a^{1-11}\right)$. b, High magnification of en face distribution of CD45+ (green) cells in the lesser curvature (LC) of adult and aged aortae. Scale bar, $20 \mu \mathrm{m}$. c, Quantification of intimal CD $45+$ cells in LC of $8 w k$ and 78wk old mice. ( $n=4$ mice per group, Mann-Whitney $T$-test, \pm SD, $p=0.0286$, two-tailed, $\left.{ }^{\star} p \leq 0.05\right)$. $d, e$ Images of

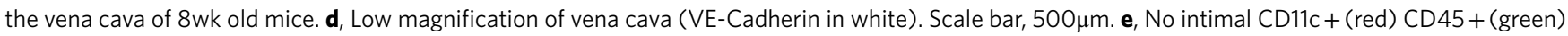
cells were found in the vena cava, even in regions of disturbed blood flow (Scale bar, 10 $\mu \mathrm{m}, \mathrm{n}=3$ mice). f, Few intimal CD $45+$ cells (green) accumulate in carotid arteries ( $8 \mathrm{wk}$ ), VE-Cadherin in red (Scale bar, $300 \mu \mathrm{m}, 50 \mu \mathrm{m}\left(\mathrm{f}^{\prime-1}\right), \mathrm{n}=8$ mice). High magnification inserts on the right showing carotid branches (disturbed flow). $\mathbf{g}$, Whole mount scans of the descending aortae (thoracic and abdominal) of 8 wk and 78 wk old C57BL/6 mice. Except for branches, few intimal CD45 + cells (green) were detected in $8 \mathrm{wk}$ old descending aortae, $\left(\mathrm{g}^{\prime}\right)$ whereas $78 \mathrm{wk}$ old mice display large number of intimal CD45 + cells ( $\left.\mathrm{g}^{\prime \prime}\right)$. Scale bar, $500 \mu \mathrm{m}$ ( $8 \mathrm{wk}$ thoracic), $700 \mu \mathrm{m}$ ( $8 \mathrm{wk}$ abdominal), $1000 \mu \mathrm{m}$ (78 wk thoracic and abdominal), and $50 \mu \mathrm{m}\left(\mathrm{g}^{1-11}\right), \mathrm{n}=5$ ( $\left.8 \mathrm{wk}\right)$ and $6(78 \mathrm{wk}) \mathrm{mice} . \mathbf{h}$, Topographic map to guide the quantification of immune cells shown in ' $\mathbf{i}$ '. $\mathbf{i}$, Intimal CD $45+$ cells per aortic region at $8 w k, 52 w k$, and $78 w k$ old mice $(n=3$ mice per timepoint, two-tailed T-test, $\pm S D, p=0.0022$ (2: $8 w k$ vs 52wk), $p=0.0031$ (2: $8 w k$ vs $78 w k), p=0.0175$ ( $2: 52 w k$ vs $78 w k$ ), $p=0.0396$ (3: $8 w k$ vs $78 w k), p=0.0052(5: 8 w k$ vs $52 w k),\left({ }^{\star} p \leq 0.05,{ }^{\star \star} p \leq 0.01\right)$. 


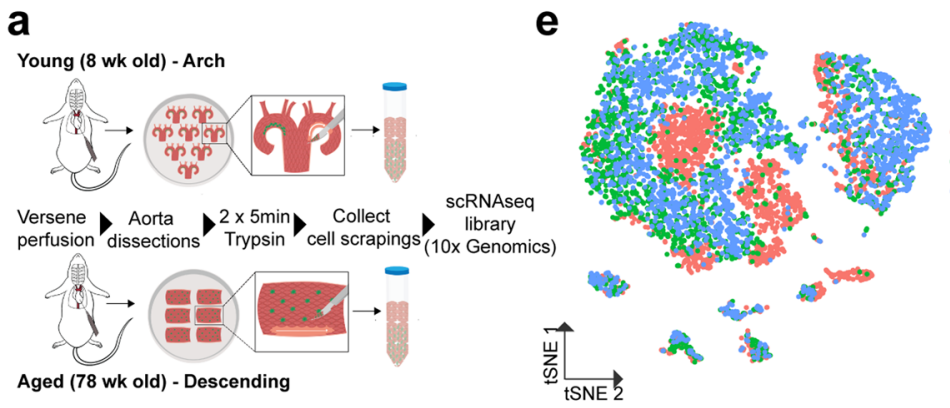

b
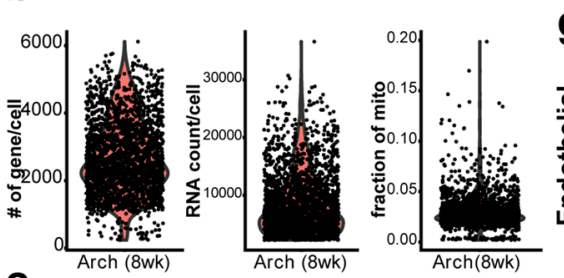

g
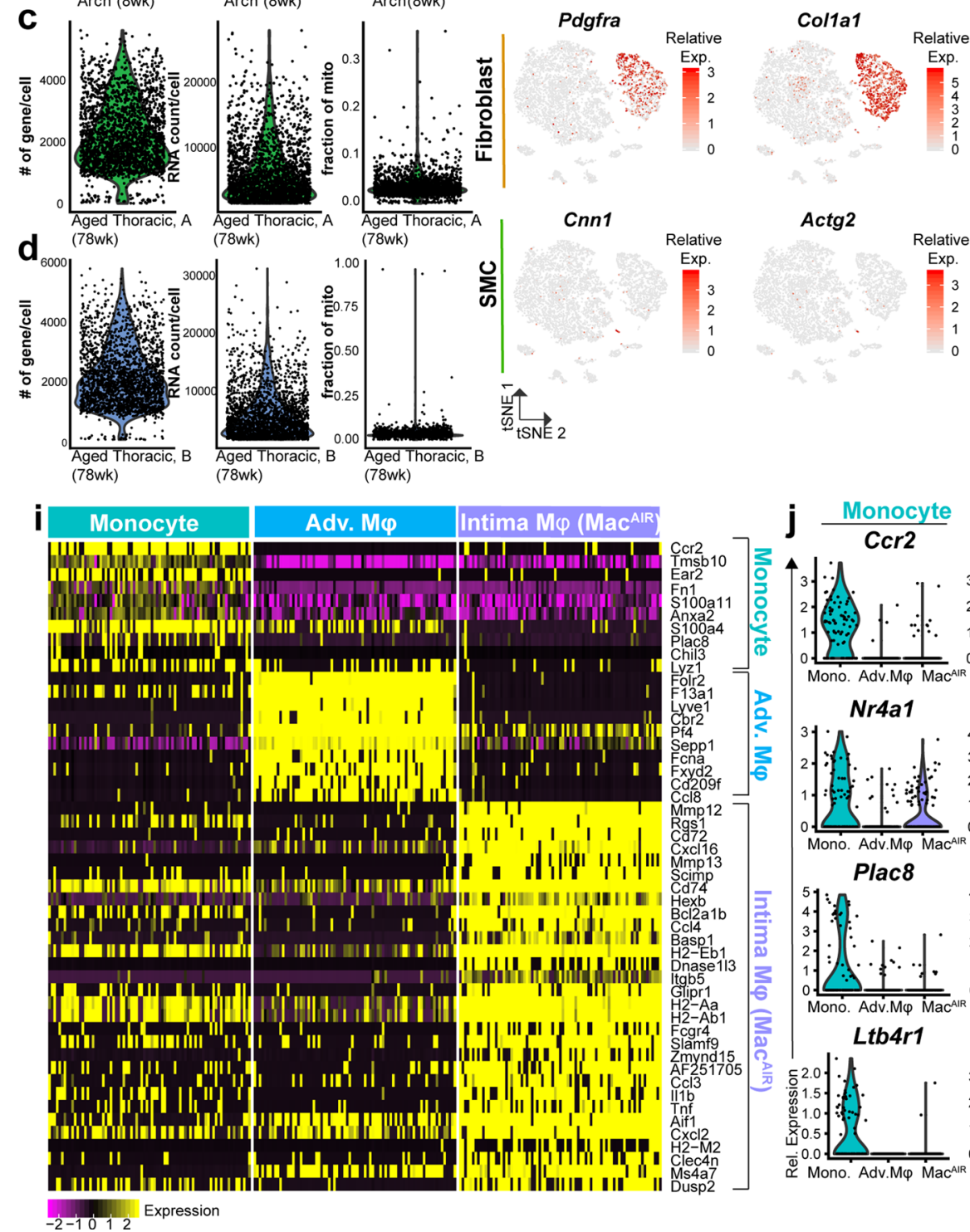

$\frac{\text { Adventitia M } \varphi}{\text { Lyve1 }}$

f

Young Arch

Aged Descen. A

Aged Descen. B

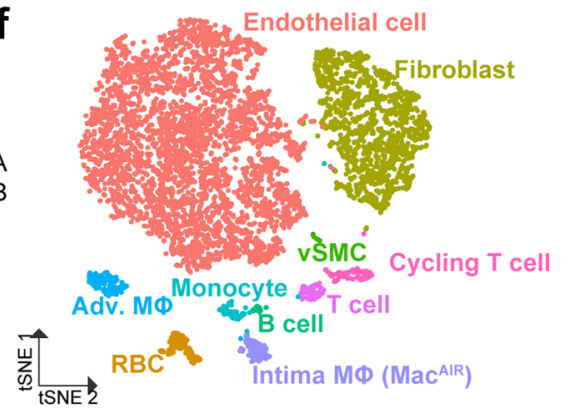

h
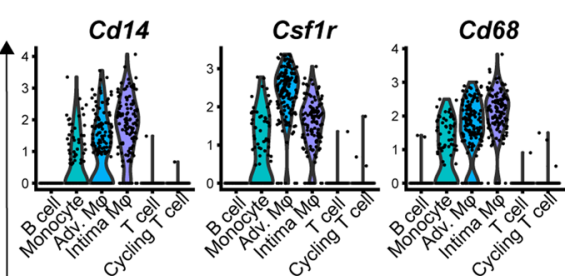

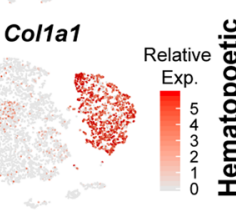

Actg 2
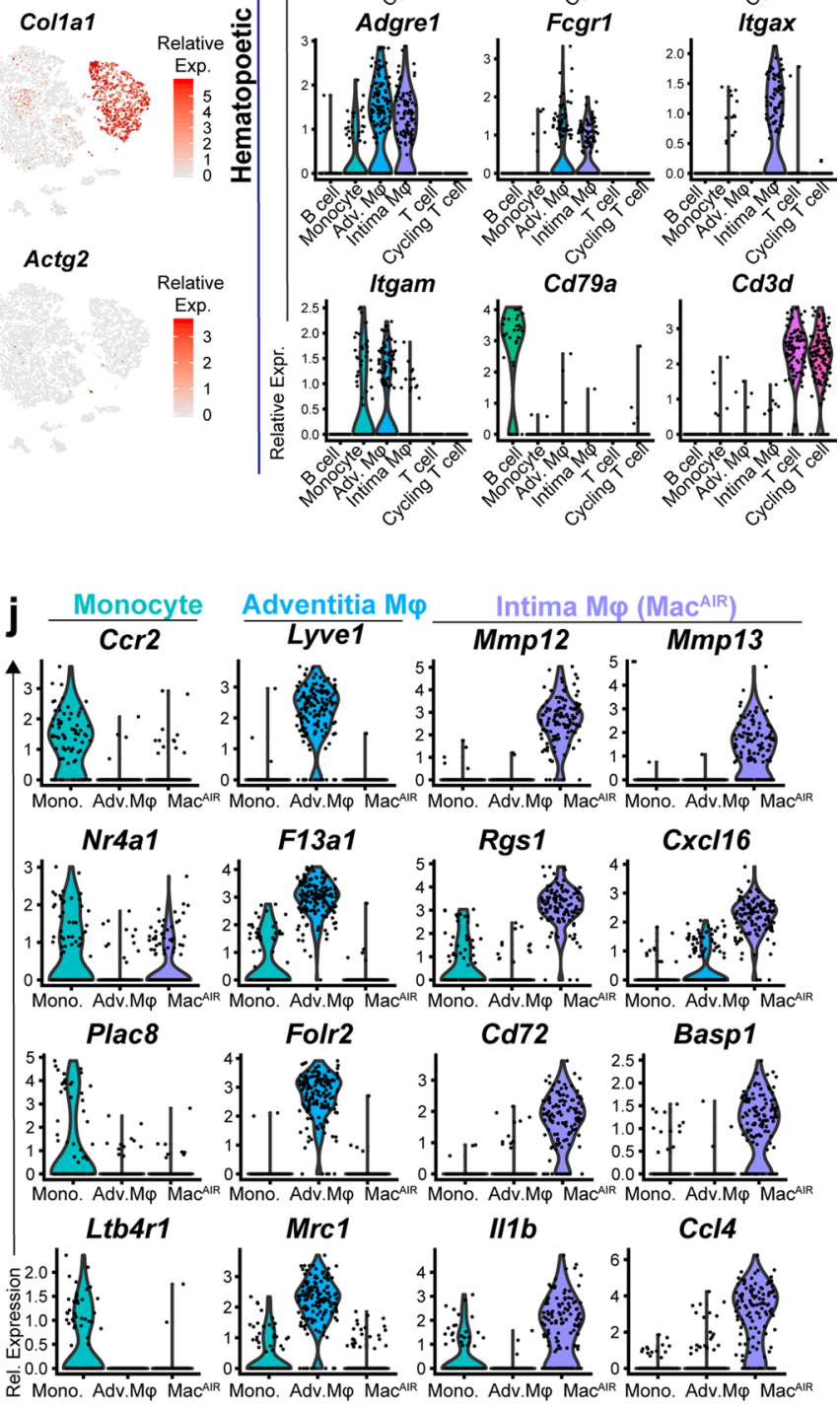

Extended Data Fig. 2 | See next page for caption. 
Extended Data Fig. 2 | scRNA-seq uncovers the macrophage identity and unique transcriptional signature of the intimal immune cell population. a, Schematic to illustrate the process for isolation of intimal, aortic cells for single-cell RNA sequencing. $\mathbf{b}$-d, Violin plots representing the Quality Control (QC) metrics for each scRNAseq library: Arch and Aged Thoracic A and B. Note, Aged Thoracic A \& B represent two independent libraries, not technical replicates, each composed of cells isolated from 6-8 aortae. Shown are: the number of genes per cell ( $n$ Gene), the absolute number of observed transcripts per cell (nUMI, Unique Molecular Identifier), and the fraction of transcripts arising from the mitochondria genome (fration of mito). $\mathbf{b}$, Aortic arch library (red); c, Aged thoracic library A (green); d, Aged thoracic library B (blue). e, tSNE plot of cells from three independent libraries: aortic arch in red ( $8 \mathrm{wk}$ ) and aged descending (descen.) A (in green) and B (in red) (78wk). 6-8 aortas were used per library. f, Ten distinct cell types were identified, and cellular identities were assigned to each cluster. $\mathbf{g}$, tSNE analysis and heat map-style representation of non-hematopoietic vascular lineage markers (color key indicates expression level). $\mathbf{h}$, Violin plots showing expression levels of the most prominent hematopoietic lineage markers shown as normalized gene expression per cell. i, Top differentially expressed genes expressed by monocytes, adventitia macrophages (Adv. M $\phi$ ), aortic intima-resident macrophages (Mac AIR) (color key indicates expression level). j, Violin plots showing expression levels of selected genes that distinguished monocytes (left column), Adv. M $\phi$ (center left column), and Mac AlR (right columns). 
a

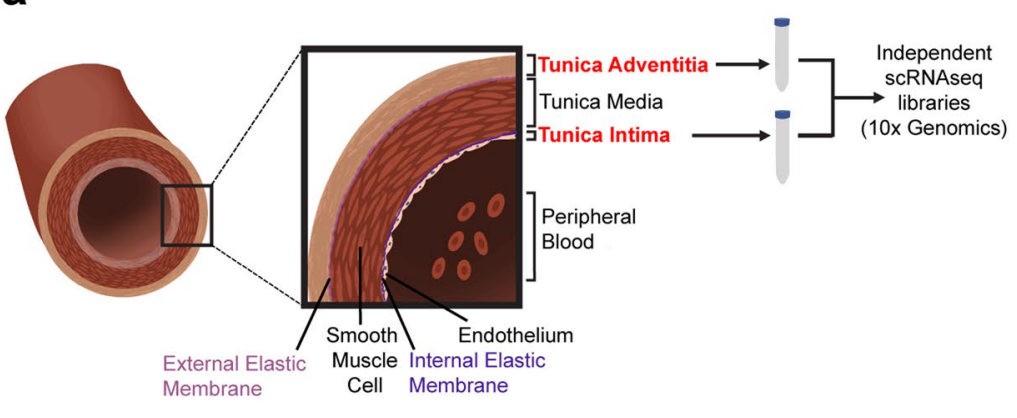

b

Aged Descen. (intima enriched), A Aged Descen. (intima enriched), B Aged Adventitia

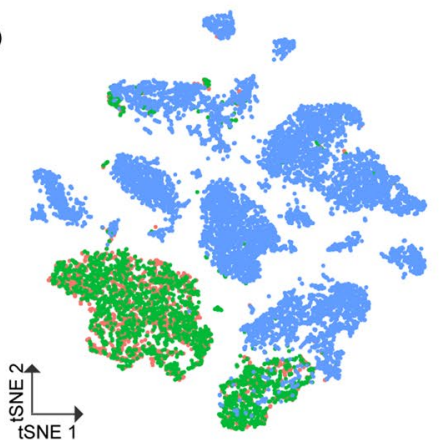

C

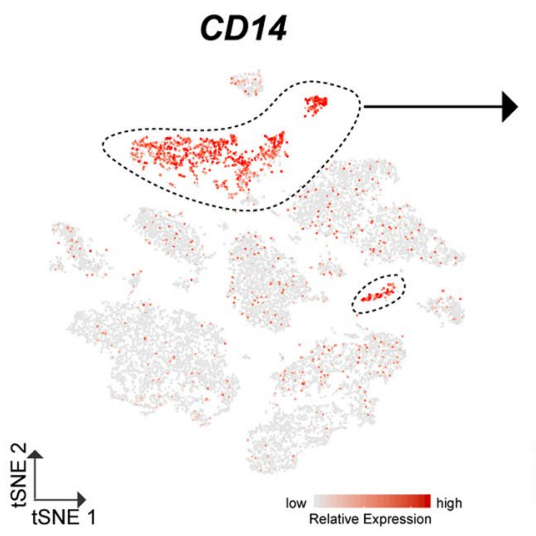

Aged Descend. (intima enriched), A Aged Descend. (intima enriched), B d Aged Adventitia

\section{CD14}

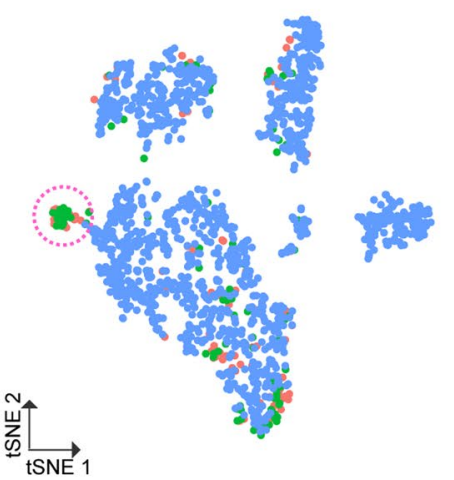

e

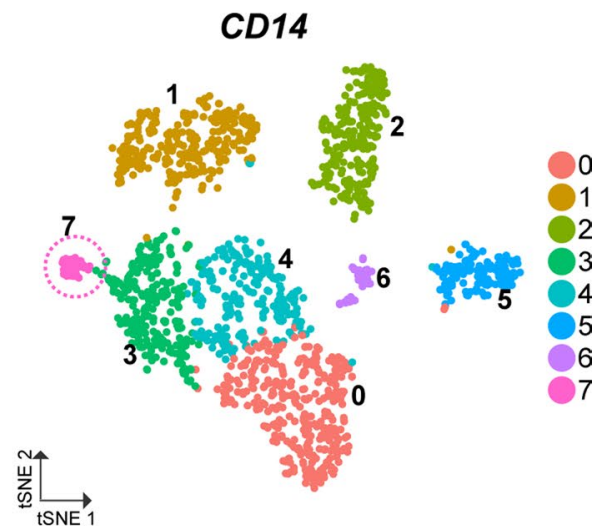

f

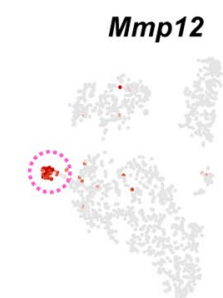

Lyve1

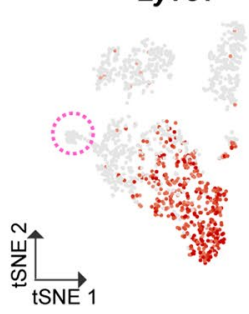

Mmp13
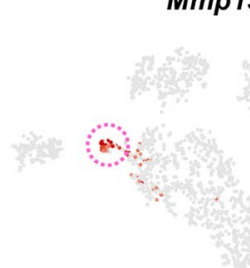

Fcgr1(CD64)

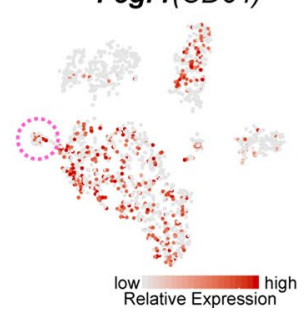

g

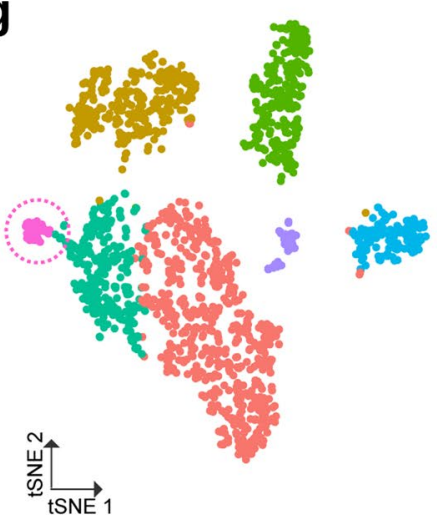

'Adventitia' Macrophage

Monocyte

Monocyte (Plac8+)

Macrophage (MHCll+)

Macrophage (Prg4+)

Fibroblast

Intima macrophage (Mac ${ }^{\mathrm{AIR}}$ )

Extended Data Fig. 3 | scRNAseq on dissected adventitia. a, Illustration displaying the different aortic layers and highlighting the tissue that was dissected and used to generate independent scRNAseq libraries for comparison: tunica adventitia vs tunica intima, (red text). b, tSNE projections of cells from three independent libraries: aged adventitia ( $78 \mathrm{wk}$ ) and aged thoracic A and B (78wk). c, Expression of CD14 to identify myeloid cells in aged thoracic and aged adventitia data sets (color key indicates expression level). d, tSNE plot displaying only the CD14 expressing cells from c. Pink dotted circle highlights a cluster of cells that are exclusively found in intimal-enriched libraries (Aged Thoracic A and B). e, tSNE plot identifying unique cell clusters presented in d. Note, cluster 7 (pink dotted cluster) is a clearly distinct population. f, Expression of Lyve1 was used to identify adventitia macrophages and Fcgr1 was used to identify macrophages. Note, Cluster 7 expresses Fcgr1 and also highly expresses Mmp12 and Mmp13 but it does not express Lyve1 (color key indicates expression level). $\mathbf{g}$, Cellular identities assigned to each cluster identified based on transcriptional expression patterns. 
a

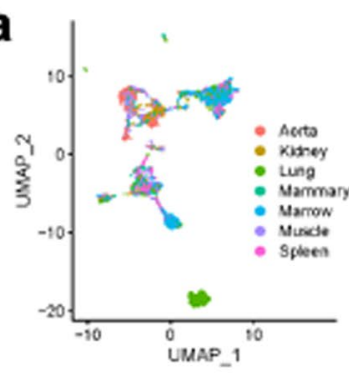

e
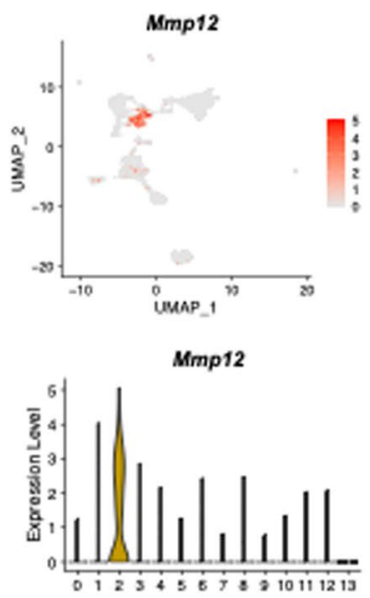

b

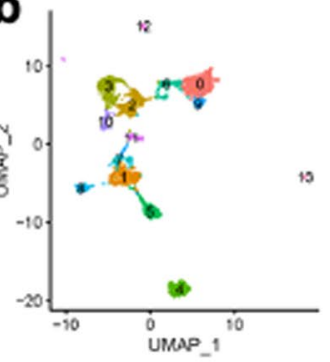

Cx3er1

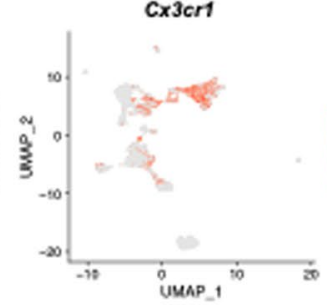

c.x3er1

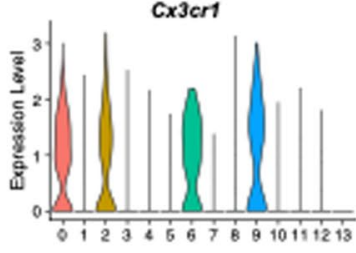

Top 50 Intima $\mathrm{M} \varphi\left(\mathrm{Mac}^{\mathrm{AIR}}\right)$ markers

f

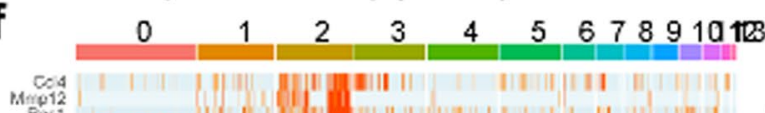

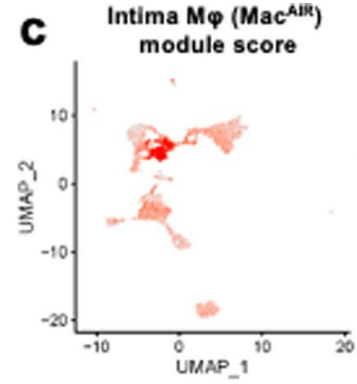

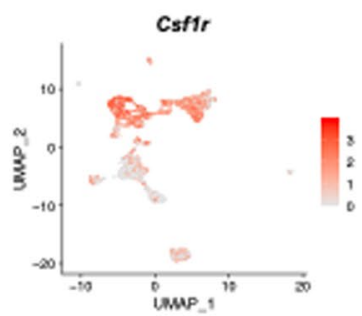

\section{g Cluster 2}
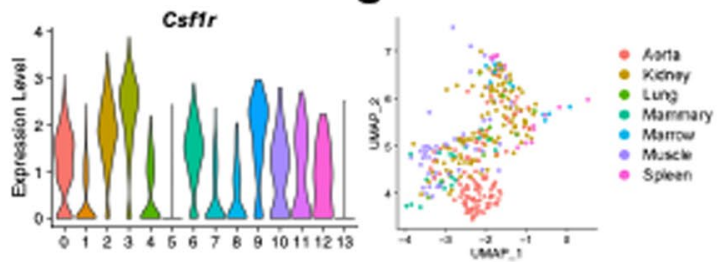

h
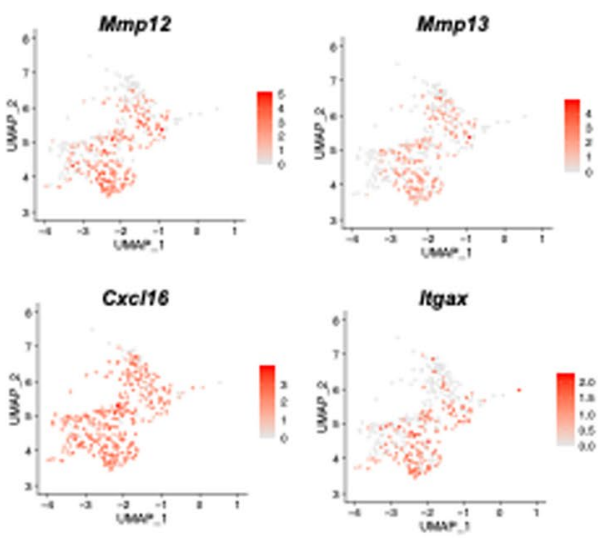

Hernandez et al. vs Chakarov et al.

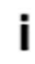

i

tyve1_llow_MHCI_high Lyve1_high_MHCII_low

\begin{tabular}{r|r|r|}
\hline Monocyte & 0.092682927 & 0.010799136 \\
\hline Adventitia macrophage & 0.001028807 & 0.229368932 \\
\hline
\end{tabular}
Intima $\mathrm{M} \phi\left(\mathrm{Mac}^{\mathrm{NR}}\right)$ 0.184177998 0.006048387

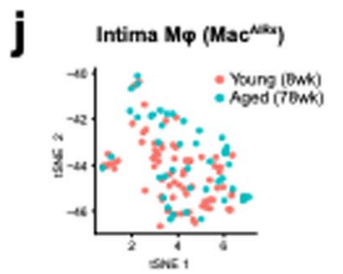

Extended Data Fig. 4 | See next page for caption. 
Extended Data Fig. 4 | Mac ${ }^{\text {AIR }}$ comparison to other published macrophage data sets. a, Uniform manifold approximation and projection (UMAP) plot of monocytes and macrophages extracted from the Tabula muris atlas and merged with the aortic intima-macrophage (Mac $\left.{ }^{A I R}\right)$ data set ('Aorta') from this work. b, UMAP plot identifying distinct cell clusters based on transcriptional signatures. c, Mac ${ }^{A I R}$ marker genes were used to calculate a module

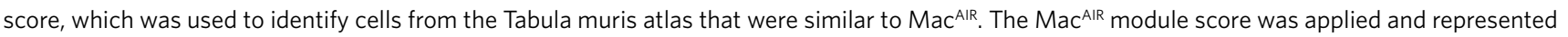
by heat map-style UMAP plot. d, Number (top graph) and percentage (bottom graph) of cells from each tissue that are in each cluster. e, UMAP heat map-style representation of MMP12 (Mac ${ }^{A I R}$ marker), Cx3cr1, and Csf1r (top row) with violin plot representation of each given gene below. $\mathbf{f}$, Heat map comparing the top 50 MacAIR markers to all the clusters found in b. $\mathbf{g}$, UMAP plotting only cluster 2 and showing tissue origin. $\mathbf{h}$, UMAP heat map-style representation of top Mac AlR markers: Mmp12, Mmp13, Cxcl16, Itgax (CD11c), i, Differential genes from the comparison among monocytes, adventitia macrophages, and Mac ${ }^{A I R}$ (our data - this study) were overlapped with genes extracted from the comparison between the Lyve ${ }^{\text {low }} \mathrm{MHCl}$ (high and Lyve ${ }^{\text {high }} \mathrm{MHClI}$ low macrophages from the Chakarov et al. dataset. The jaccard index between each pair for cell types from the two datasets were calculated and plotted in the heatmap. $\mathbf{j}$, Comparison of Mac AlRs identified in the tunica intima-enriched young arch vs aged descending data sets. 
P7

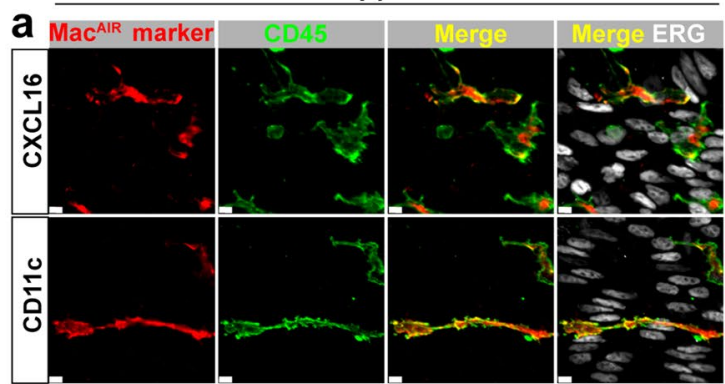

Adventitia

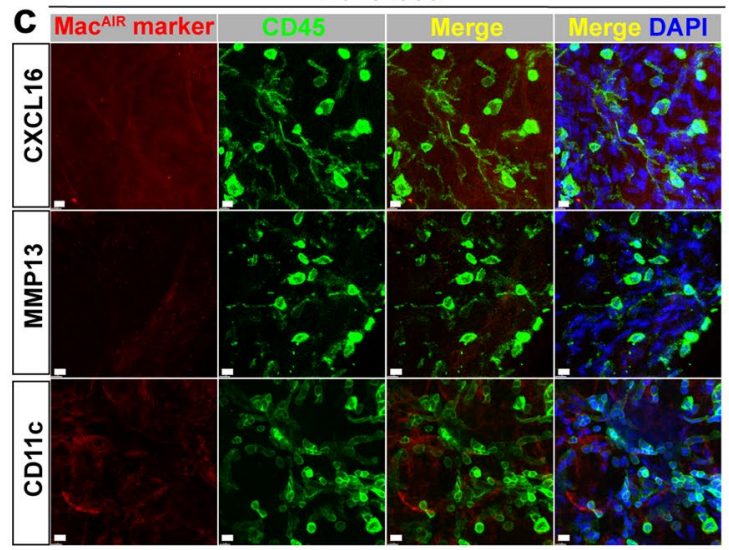

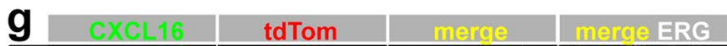

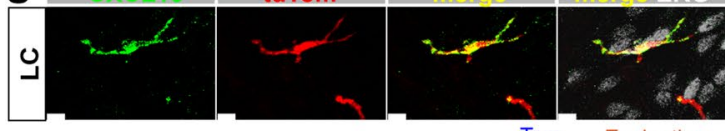

h CX3CR1-creERT2;R26-tdTom $\quad \underset{\mathrm{Ag} 1 \mathrm{P} 3}{\frac{\mathrm{Tam}}{\mathrm{T}}}$ Evaluation

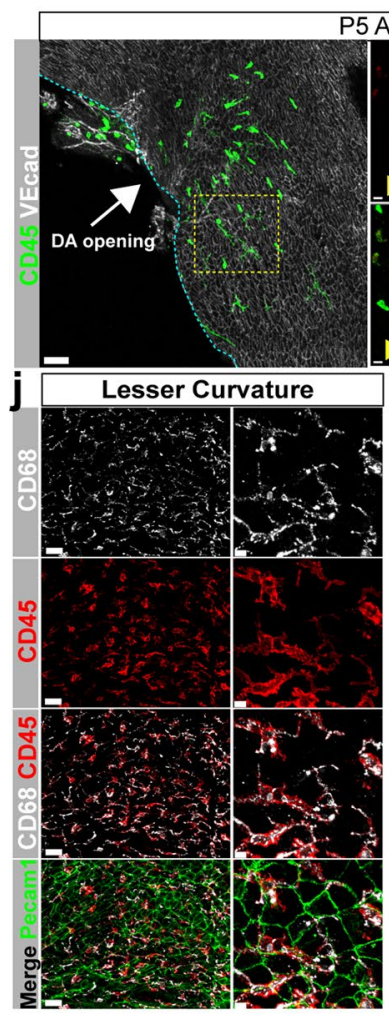

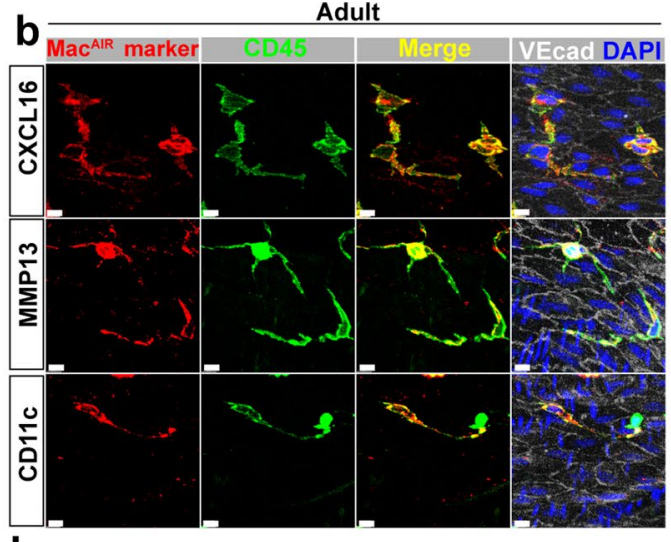

e

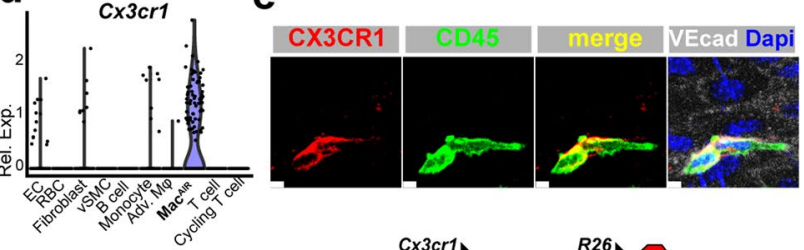

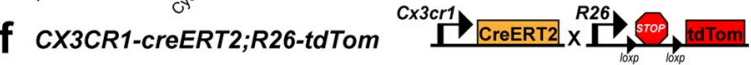

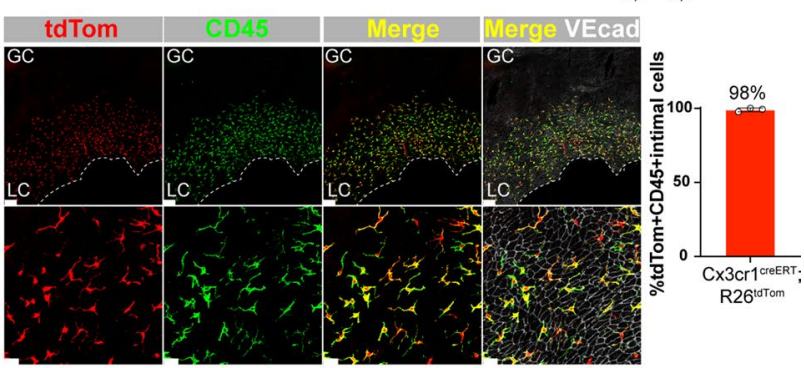

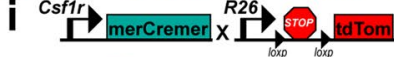
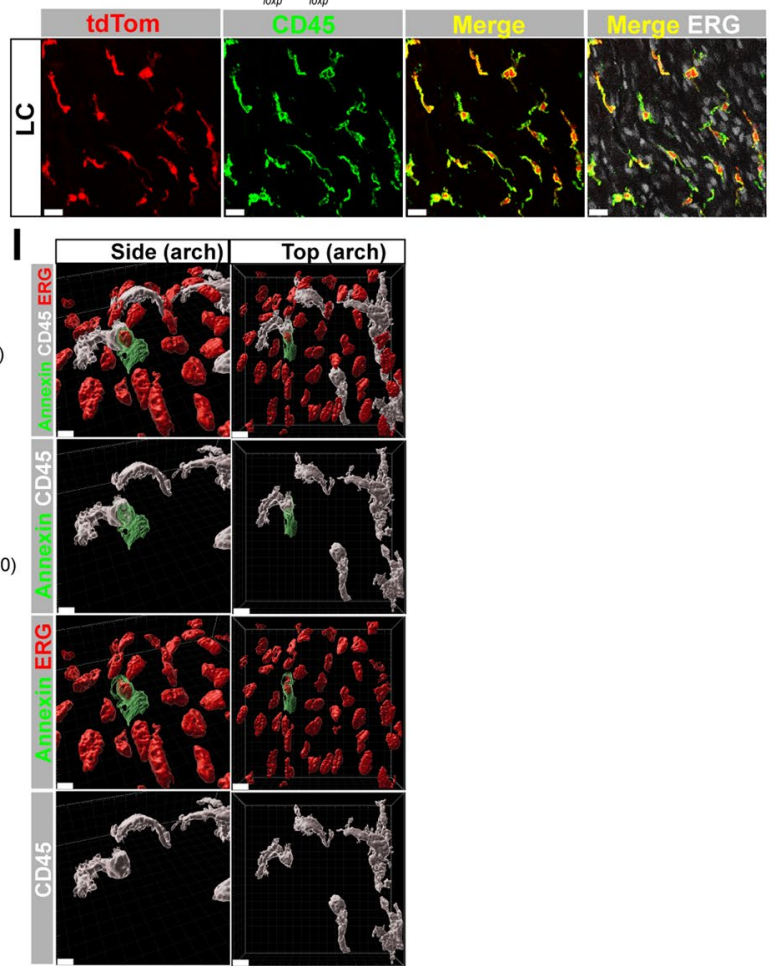

Extended Data Fig. 5 | See next page for caption. 
Extended Data Fig. 5 | Validation of Mac AIR markers identified by scRNAseq. a, b, Validation of unique Mac ${ }^{\text {AlR }}$ genes at P7 (a) and adult (b) aortae. Mac ${ }^{\text {AIR }}$ markers in red (CXCL16 MMP13 and CD11c) and CD45 in green. Scale bar, $5 \mu \mathrm{m}$ and 10 $\mu \mathrm{m}, \mathrm{n}=3$ (P7), 5 (adult) mice. c, Evaluation of the same markers as in panels a and $\mathbf{b}$, but now in the adventitia. Note that no CD45 + cells in adult adventitia expressed the Mac ${ }^{\text {AIR }}$ markers CXCL16, MMP13, and CD11c. Scale bar, $10 \mu \mathrm{m} ; \mathrm{n}=3$ mice per marker. $\mathbf{d}$-e, Mac ${ }^{\text {ARs }}$ express $\mathrm{C} \times 3 \mathrm{cr} 1$ at both the (d) transcript level and (e) protein level (in red) (Scale bar, $5 \mu \mathrm{m}$, $\mathrm{n}=3$ mice). $\mathbf{f}, \mathrm{C} \times 3 \mathrm{cr} 1$-reporter ( $\mathrm{C} \times 3 \mathrm{cr}$ r $^{\text {creERT2}} ; \mathrm{R} 26^{\text {tdTomato }}$ ) showing tdTomato reporter expression in intimal immune cells one-day post-tamoxifen (Scale bar, $100 \mu \mathrm{m}$ and $30 \mu \mathrm{m}) .98 \%$ of intimal CD $45+$ cells in the lesser curvature (LC) of adult Cx3 $\mathrm{cr}^{\text {1 }}{ }^{\text {creeRT2}} ; \mathrm{R} 26^{\text {tdTomato }}$ mice were tdTomato + ; represented on adjacent graph ( $n=3$ mice, \pm SD, GC, greater curvature). $\mathbf{g}$, Intimal tdTomato+ cells colocalize with Mac AIR marker Cxcl16, post-tamoxifen injection

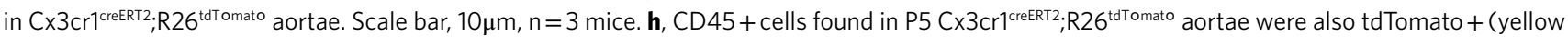

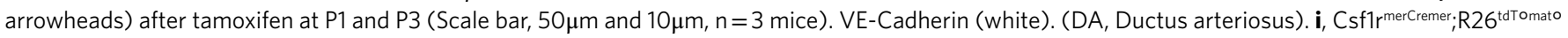
macrophage fate-mapping. Intimal CD45+cells in the lesser curvature (LC) labeled by tdTomato. Adult mice received a single injection of tamoxifen. ERG (white) (Scale bar, 20 $\mathrm{mm} ; \mathrm{n}=2$ mice). j, Images co-staining for CD68 (white), CD45 (red) and VE-Cadherin (green). Scale bar, 30 $\mu \mathrm{m}$ and $7 \mu \mathrm{m}, \mathrm{n}=3 \mathrm{mice}$. k, Expression of classical dendritic cell (DC) markers (Zbtb46, Cd8a, Ccr7) and macrophage (M $\varphi$ ) markers (Fcgr1, Adgre1, Cd68) in adventitia macrophages (Adv. M $\varphi$ ), intima-resident macrophages (Mac AlRs), and monocytes from scRNA-seq data in Extended Data Fig. 2. I, 3D surface rendering of an intimal CD45 + cell (white) phagocytosing an annexin + (green) endothelial cell (ERG +, red) in the LC. Scale bar, 10 $\mu \mathrm{m}$. 
b $\underbrace{\text { Time }}_{0 \mathrm{hr}}$ bdU inj. Harvest $^{\mathrm{hr}}$

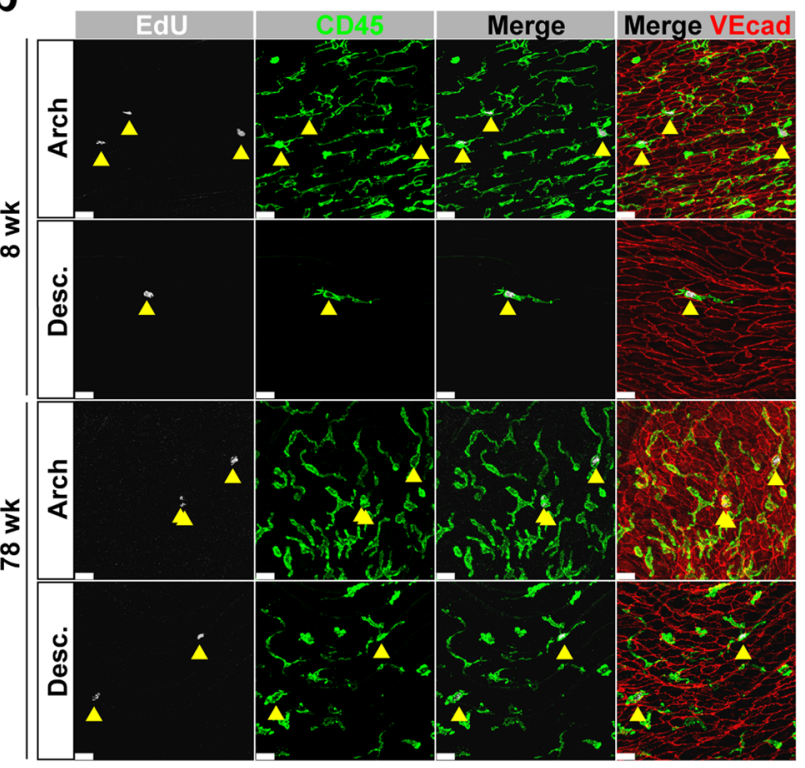

f

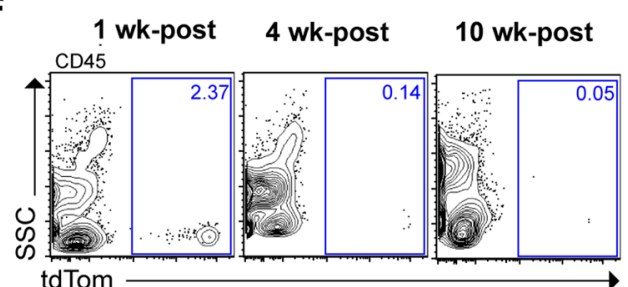

i

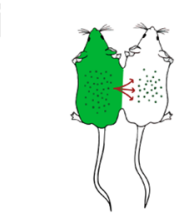

$\overbrace{\text { Surgery Evaluation }}^{10 w k s}$ contribution
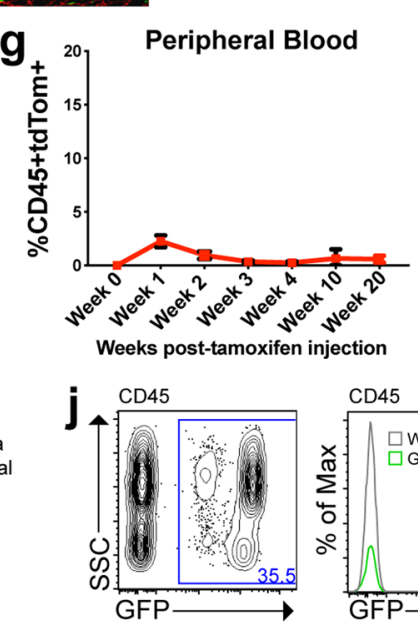

\section{d CX3CR1-CreERT2;R26-tdTOM}

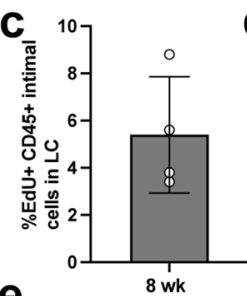

e
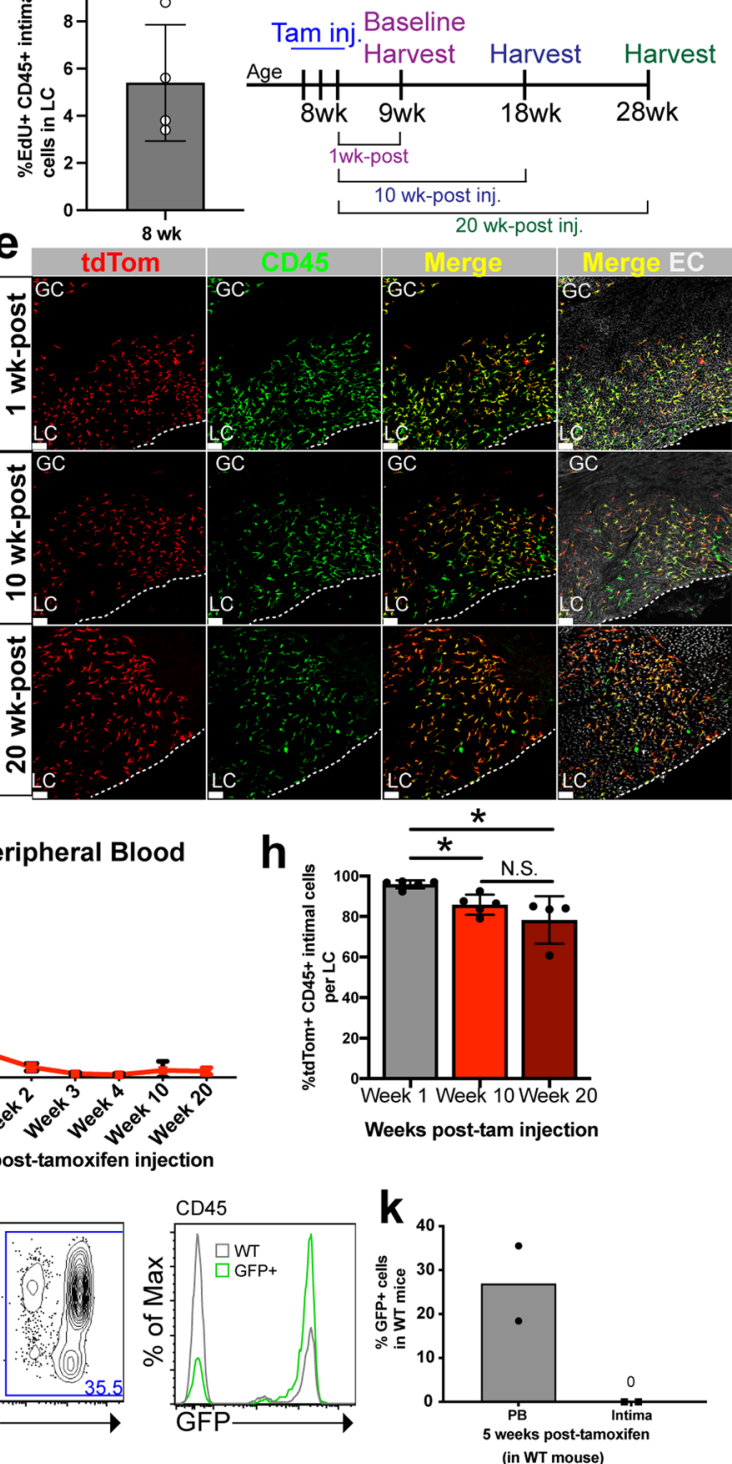

CX3CR1-CreERT2;R26-Rainbow

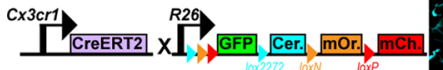
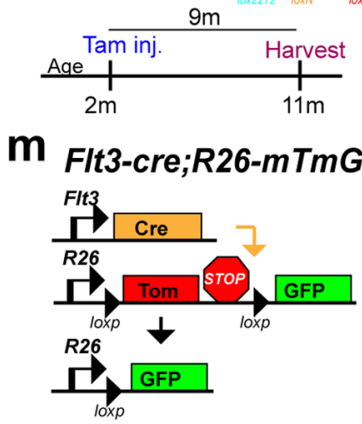

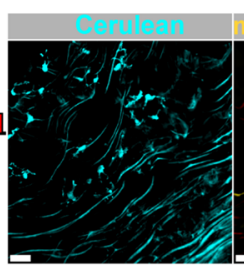

$n$

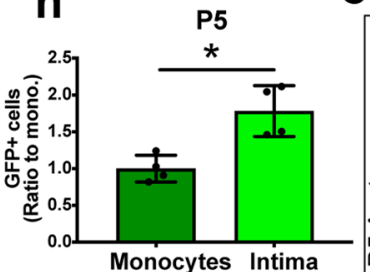

O

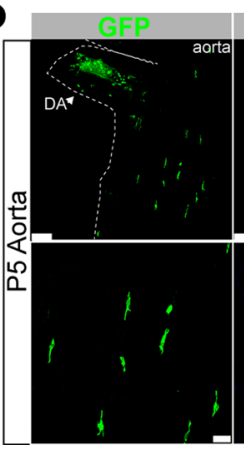

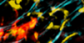
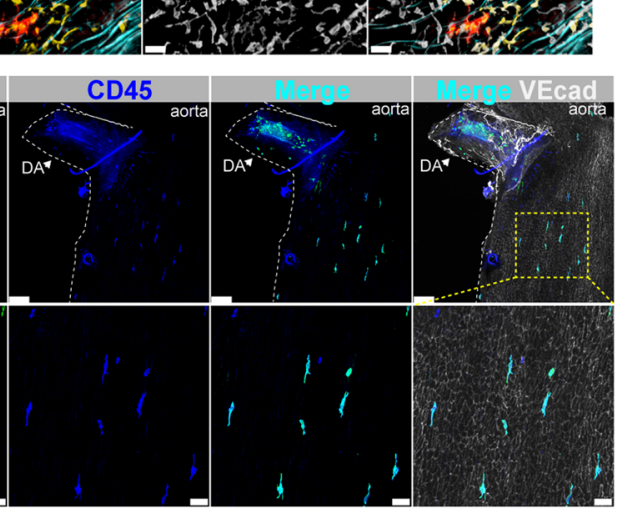

Extended Data Fig. 6 | See next page for caption. 
Extended Data Fig. 6 | Aortic intimal macrophages are a self-replicating cell population of the tunica intima residing in areas of disturbed flow. a, Timeline of 5-ethynyl-2'-deoxyuridine (EdU) incorporation. 8wk and 78wk old mice were injected with EdU and harvested 2-hrs later. b, EdU + (white) and CD45 + (green) co-expressing cells (yellow arrowheads) (arch and descending=Desc.). Scale bar, 20 $\mu \mathrm{m}, \mathrm{n}=3$ (78wk), 8 ( $8 \mathrm{wk}$ ) mice. c, Intimal EdU $+C D 45+$ cells found in $L C$ at $8 w k(n=4$ mice, $\pm S D)$. d, Experimental design for lineage tracing. 8 wk old $C \times 3 c r 1$ creeRT2; R26 $6^{\text {tdomato }}$ mice were pulselabeled by Tam injection (inj.). Mice were harvested at 1wk-post-tam to establish reporter baseline. Evaluation was done at 10- and 20-wks post-tam. e, Images of CD45 (green) and tdTomato+ at 1-, 10-, and 20-wks post-tamoxifen. Endothelial cell (EC) labeled with VE-cadherin (1- and 10-wk) and ERG (20-wk). Scale bar, 80 $\mu \mathrm{m} ; \mathrm{n}=4$ (20wk), 5 (1wk,10wk) mice. GC = greater curvature. f, Representative fluorescence activated cell sorting (FACS) plot of

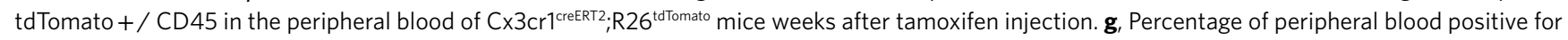
tdTomato during the time course of the study post-tamoxifen measured by flow cytometry ( $n=3$ ( $2 w k, 3 w k, 4 w k, 10 w k), 5(20 w k)$, and 6 (1wk) mice,

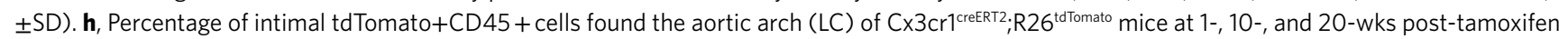
injection ( $n=4$ (20wk), 5 (1wk, 10wk) mice, Mann-Whitney t-test, \pm SD, $p=0.0159$ (1wk vs 10wk), p=0.0159 (1wk vs 20wk), two-tailed, * $p \leq 0.05)$. i, Experimental design and timeline indicating the possible outcomes of the parabiosis experiment. GFP + and wild-type (WT) mice were surgically paired together to share chimeric circulation for 5 wks prior to analyses. j, Representative FACS plot and histogram showing blood chimerism after parabiosis. $\mathbf{k}$, Quantification of intimal GFP $+C D 45+$ cells found in the $L C$ of the WT mice $(n=2$ pairs of mice, \pm SD). PB = peripheral blood; WT $=$ wild-type. $\mathbf{l}$, Clonal tracing model, design, and possible outcomes. 2 month old Cx3cr1 creeRT2;R26 Rainbow were administered a single dosage of tamoxifen. At 11 months (9 months later), aortae were evaluated clonal expansion and retention of labeling of Mac ${ }^{\text {AlRs }}$ overtime. Images revealed clones of Mac ${ }^{\text {AlRs }}$ labeled and CD45 (white)

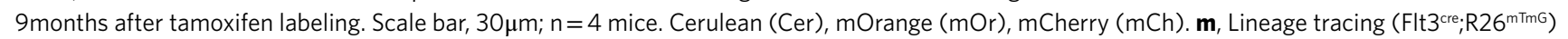
that labels cells derived from definitive hematopoiesis. Flt3-driven cre recombinase (cre) expression leads to an irreversible switch from Tomato (tom) to GFP expression in Flt3-expressing cells. As this is an irreversible switch, progeny will also be labeled GFP. $\mathbf{n}$, GFP expression in CD45+cells of P5 Flt $3^{\text {cre }}, R 26^{\mathrm{mTmG}}$ aortae relative to circulating monocytes from the same mice $\left(n=4\right.$ mice, Mann-Whitney $t$-test, $\left.\pm S D, p=0.0286,{ }^{\star} p \leq 0.05\right)$. $\mathbf{0}, I_{m a g e s}$ of P5 Flt3 ${ }^{\text {cre }}$ :R2 ${ }^{\mathrm{mTmG}}$ aortae showing GFP expression (green) of intimal CD45+ (blue) cells exiting the ductus arteriosus (DA) $(n=4$ mice). 
a

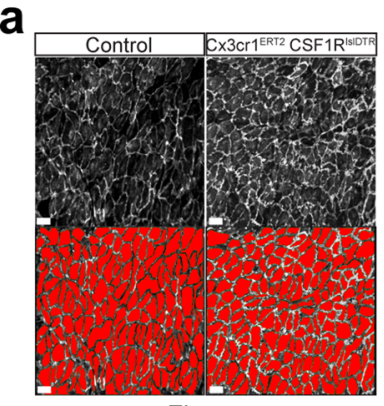

C
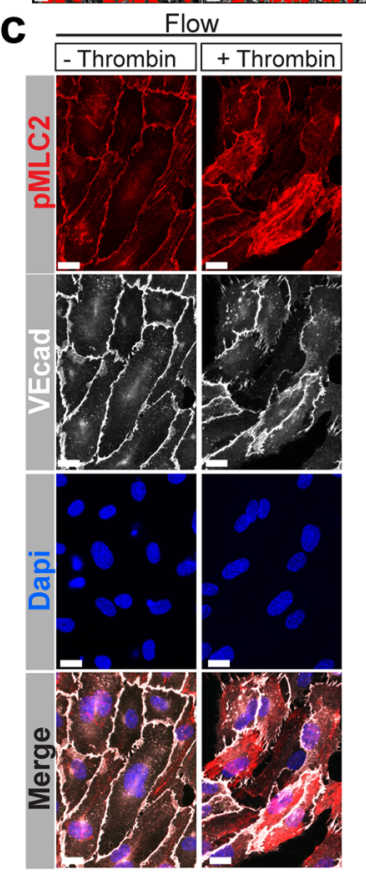

f

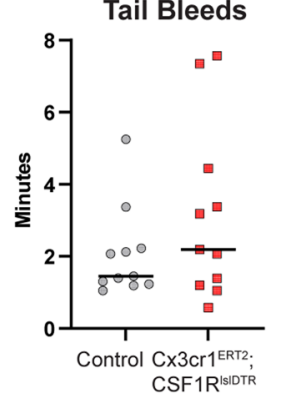

h

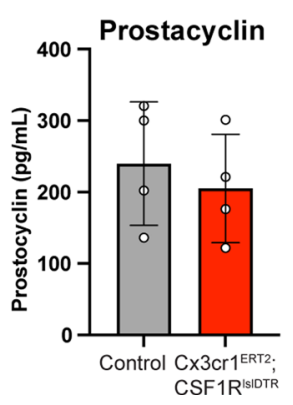

b
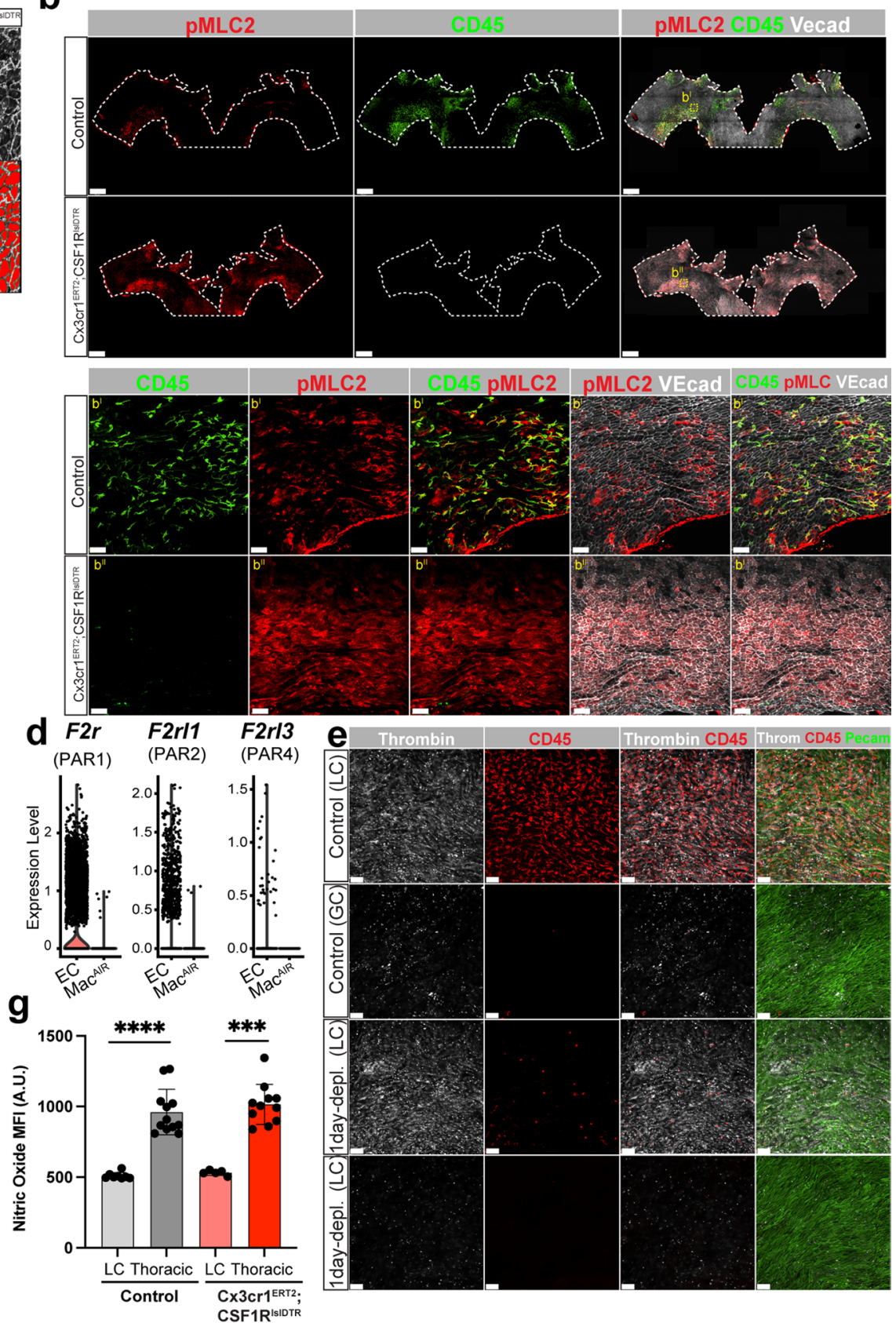

Extended Data Fig. 7 | See next page for caption. 
Extended Data Fig. 7 | Thrombin distribution and signaling in areas of disturbed flow. a, VE-cadherin (white) of control and macrophage-depleted (CX3 $\mathrm{Cr}^{\text {creeRT2} ; C S F 1 R ~}{ }^{\text {IIDTR }}$ ) aortae (upper panel). Changes in cell area and cell number were quantified by filling in red the area limited by VE-Cadherin

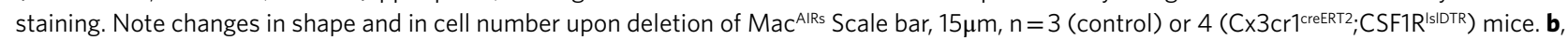
Low (upper panels) and high (lower panels) magnifications of control and CX3cr1 ${ }^{\text {creeRT2 }}$;CSF1R ${ }^{\text {ISDTR }}$ aortae staining for phosphorylation of myosin light chain 2 (pMLC2) (in red) and CD45 (green) one day post-diphtheria (DTx) toxin injection. Scale bar, 500 $\mu$ m (upper panels), 50 $\mu \mathrm{m}$ (lower panels); $\mathrm{n}=5$ mice per group. $\mathbf{c}$, Effect of thrombin on human aortic endothelial cells (HAECs) pMLC2 (red) cultured under laminar flow. VE-cadherin (white) provides information on cell shape. DAPI (blue). Scale bar, 10 $\mu$ m. d, F2r (PAR1), F2rl1 (PAR2), and F2rl3 (PAR4) expression in endothelial cells (EC) and in Mac AlRs from the arch scRNAseq data sets (Extended Data Fig. 2). e, En face images of the lesser curvature (LC) and greater curvature (GC) staining

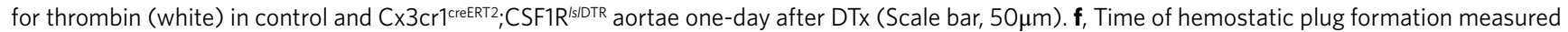
following transverse amputation of the tip of the tail $(3 \mathrm{~mm})$ to assess hemostasis function in control and CX3 $\mathrm{cr}^{\text {creeRT2 }}$; CSF1R ${ }^{\text {ISITR }}$ mice one-day post-DTX injection ( $n=11$ mice per group). $\mathbf{g}$, Ex vivo mean fluorescent intensity (MFI) measurements of nitric oxide in the lesser curvature (LC) and thoracic aorta from control and $\mathrm{C} \times 3 \mathrm{cr}^{\text {creeRT2}} ; \mathrm{CSF1R}{ }^{\text {IsITR }}$ mice one-day post-DTx injection $(\mathrm{n}=2$ mice per group and 3-7 number of areas quantified per mouse; MannWhitney $T$-test, \pm SD, $p=0.0005$, two-tailed ${ }^{* \star * \star} p \leq 0.0001$ ). DAF-FM Diacetate (4-Amino-5-Methylamino-2',7'-Difluorofluorescein Diacetate) was used to detect and quantify NO. h, Plasma levels of prostacyclin in control and Cx3cricreeRT2;CSF1R ${ }^{\text {IsITR }}$ one-day post-DTx injection, measured by ELISA. No statistically significant changes noted $(n=4$ mice per group, Mann-Whitney $t$-test, \pm SD). 


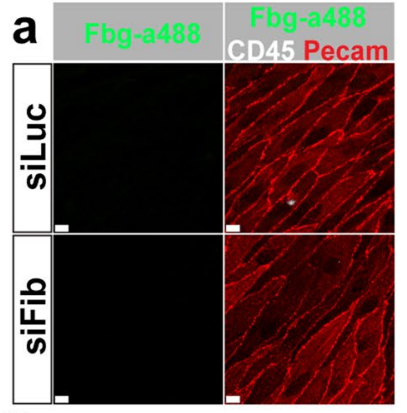

Blood Flow $\longrightarrow$

C

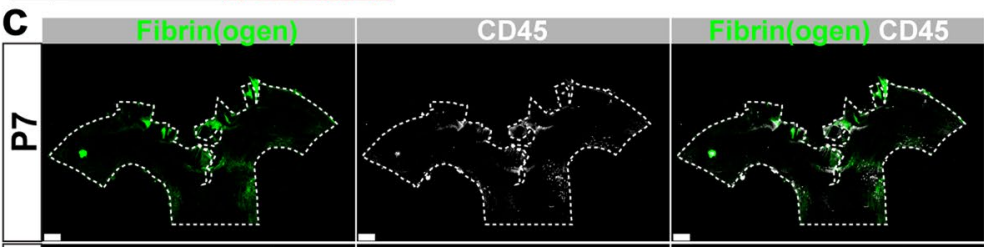

魰
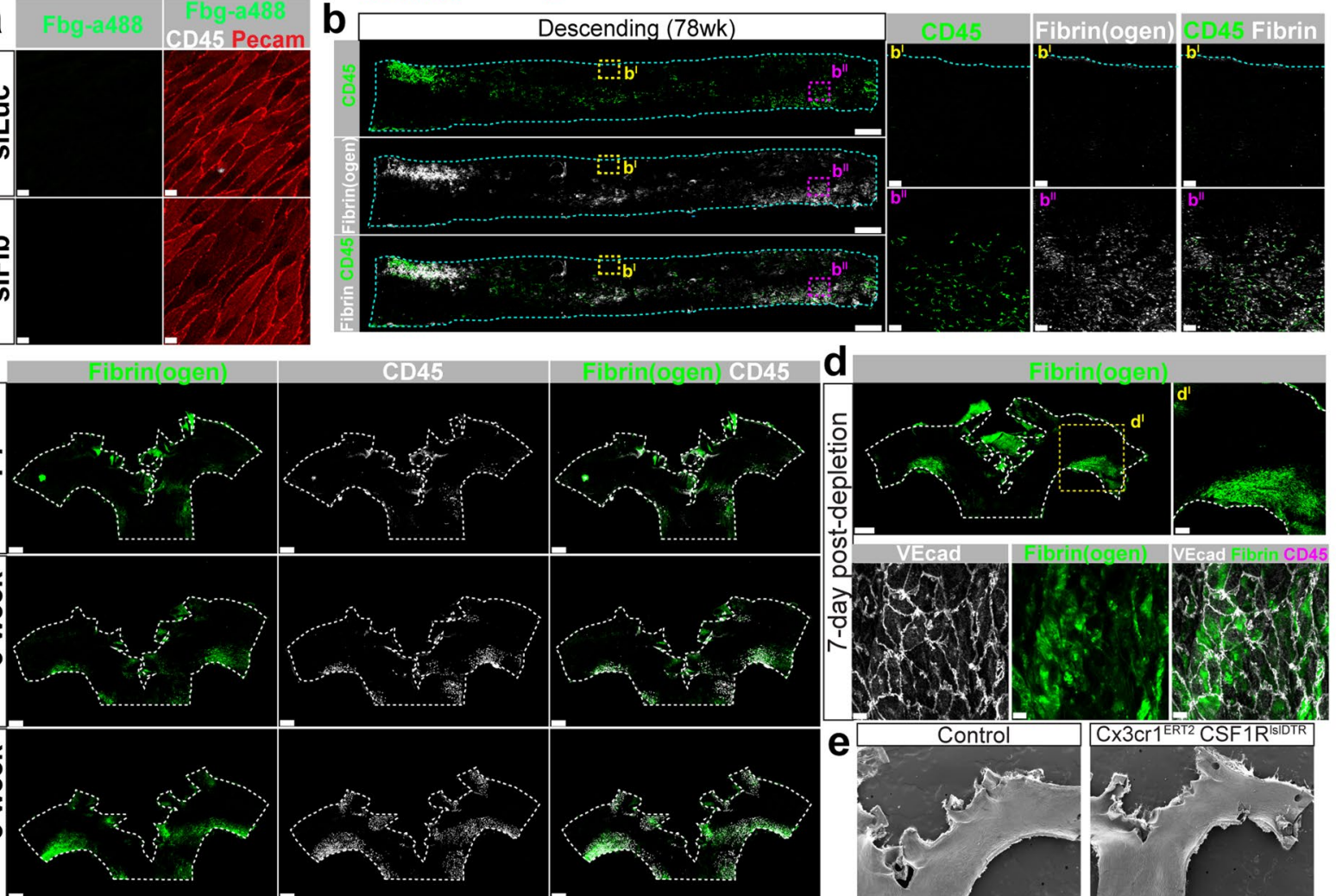

\section{d}

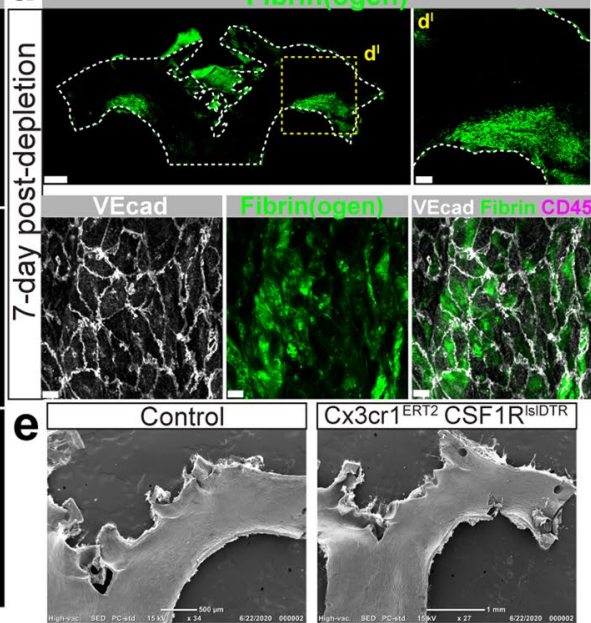

f
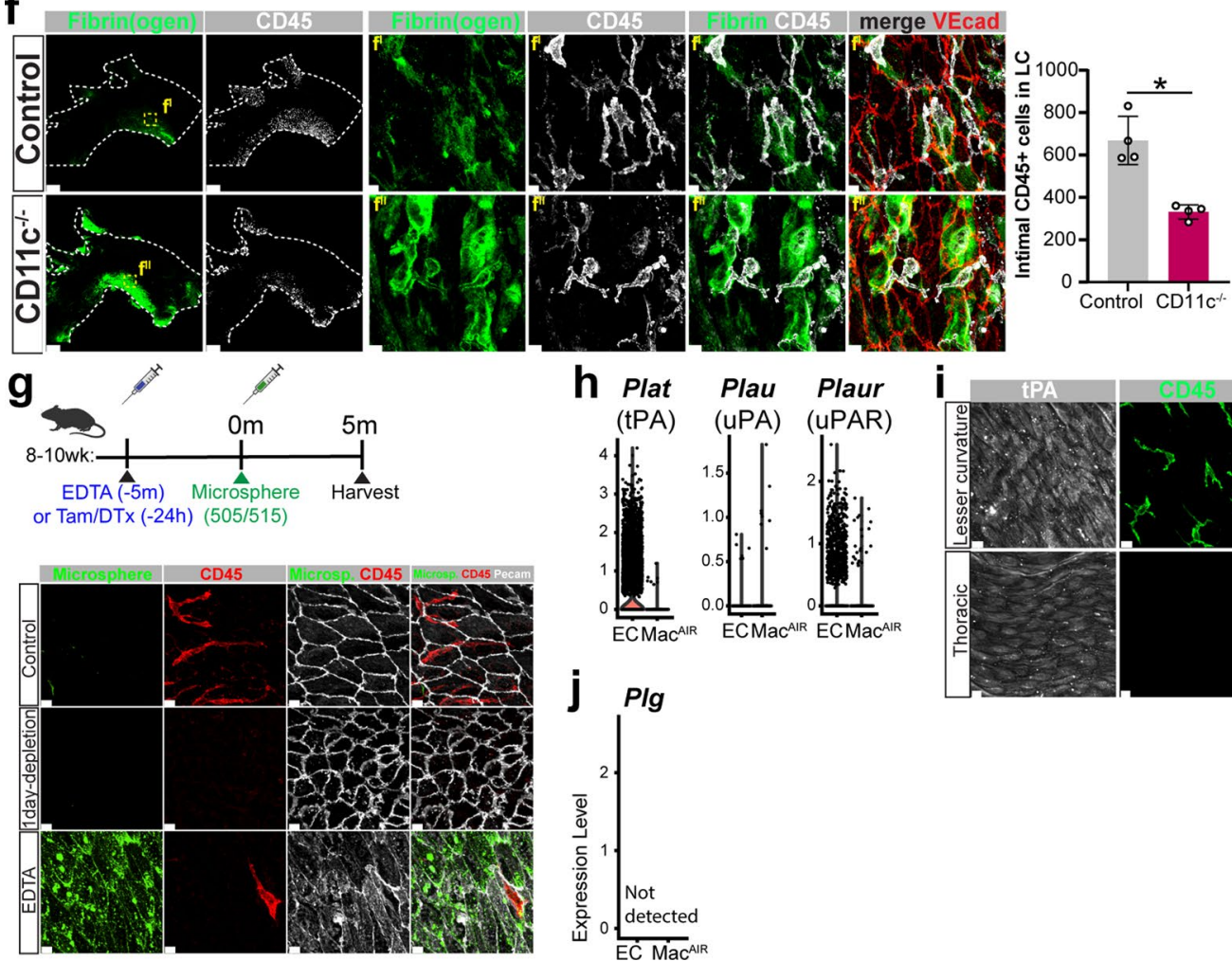

$\begin{array}{rrr}\text { Plat } & \text { Plau } & \text { Plaur } \\ \text { (tPA) } & \text { (UPA) } & \text { (UPAR) }\end{array}$

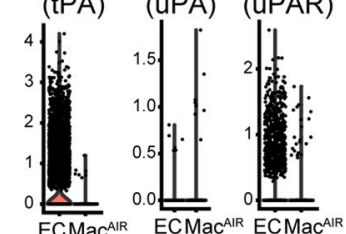

j $P l g$
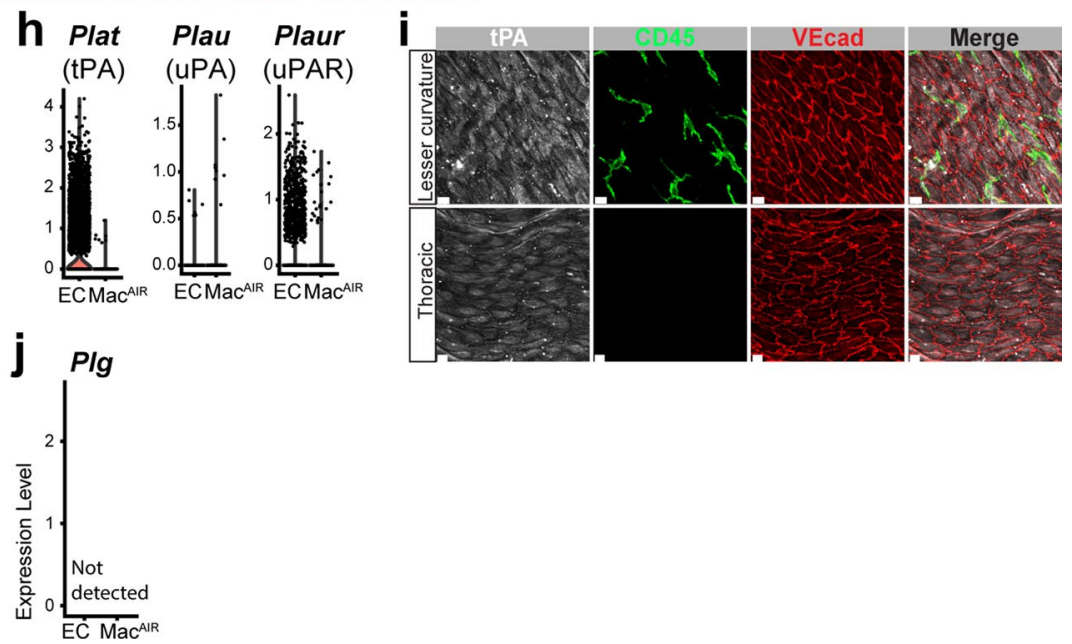

Extended Data Fig. 8 | See next page for caption. 
Extended Data Fig. 8 | Mac ${ }^{\text {ARs }}$ are necessary to prevent fibrin accumulation in areas of turbulent flow. a, Images of the greater curvature after treatment with siFibrinogen or siLuciferase (control) for 7 days and then exposed to Fbg-a488 intravenously for 3 hrs. No fibrinogen binds to the greater curvature (unlike Fig. 5f). Scale bar, $7 \mu \mathrm{m} ; \mathrm{n}=4$ mice per group. b. Descending aortae (78wk old) stained for fibrin(ogen) (white) and CD45 (green). Higher magnifications in $b^{\prime}$ and $b^{\prime \prime}$, right panels. Scale bars, $1000 \mu \mathrm{m}$ and $100 \mu \mathrm{m}\left(b^{1-1}\right), n=3$ mice. c, Time-course of fibrin(ogen) (in green) and CD45 (white) at P7 ( $n=3$ mice), $3 w k\left(n=5\right.$ mice), and $8 w k\left(n=20\right.$ mice) old mice. Scale bar, 300 $\mu$ m. d, Fibrin(ogen) (green) in Cx3cr1 creeRT2; CSF1R ${ }^{\text {slIDTR }}$ 7-days post-macrophage depletion. Scale bar, $500 \mu \mathrm{m}, 150 \mu \mathrm{m}(\mathrm{d}$ '), and $8 \mu \mathrm{m}$ (bottom row). e, Scanning electron microscopy of aortae from control and Cx3 $\mathrm{Cr}^{\text {creeRT2 }} ; \mathrm{CSF}^{\text {IsIDTR }}$ 14-days post-macrophage depletion ( $\mathrm{n}=3$ mice per group). $\mathbf{f}$, Images of the aortic arch comparing fibrin(ogen) (green) and CD45 (white) in Control and CD11 $c^{-1}$ mice (Scale bar, 300 $\mathrm{m}$ and $8 \mu \mathrm{m}, \mathrm{n}=4$ mice per group). Higher magnifications (f' and f') on the right. Graph with number of CD $45+$ cells in the LC of control and CD11 $c^{-1-}$ mice. $\left(n=4\right.$ mice per group, Mann-Whitney T-test, $\pm S D, p=0.0286$, two-tailed, $\left.{ }^{*} p \leq 0.05\right)$. g, Schema of

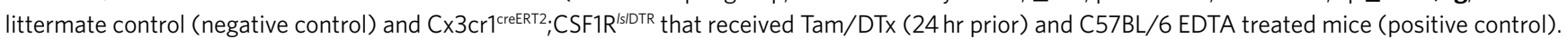
High magnification shown below. VE-Cadherin (white), CD45 (red) (Scale bar, $7 \mu \mathrm{m} ; \mathrm{n}=3$ mice per group). h, Plat (tPA), Plau (urokinase), and Plaur (urokinase receptor) expression in endothelial cells (EC) and Mac AIRs from data sets in Extended Data Fig. 2. i, Immunostaining for tPA (white) in the LC and in the thoracic aorta in relation to CD45 (green). Endothelial cells (red) (Scale bar, $10 \mu \mathrm{m} ; \mathrm{n}=3 \mathrm{mice}$ ). $\mathbf{j}$, Expression of plasminogen (Plg) undetected in EC and Mac AlRs by scRNA-seq. 


\section{Reporting Summary}

Nature Portfolio wishes to improve the reproducibility of the work that we publish. This form provides structure for consistency and transparency in reporting. For further information on Nature Portfolio policies, see our Editorial Policies and the Editorial Policy Checklist.

\section{Statistics}

For all statistical analyses, confirm that the following items are present in the figure legend, table legend, main text, or Methods section.

$\mathrm{n} / \mathrm{a} \mid$ Confirmed

$\bigotimes$ The exact sample size $(n)$ for each experimental group/condition, given as a discrete number and unit of measurement

\ A statement on whether measurements were taken from distinct samples or whether the same sample was measured repeatedly

The statistical test(s) used AND whether they are one- or two-sided

Only common tests should be described solely by name; describe more complex techniques in the Methods section.

Х A description of all covariates tested

Х A description of any assumptions or corrections, such as tests of normality and adjustment for multiple comparisons

A full description of the statistical parameters including central tendency (e.g. means) or other basic estimates (e.g. regression coefficient)

AND variation (e.g. standard deviation) or associated estimates of uncertainty (e.g. confidence intervals)

For null hypothesis testing, the test statistic (e.g. $F, t, r$ ) with confidence intervals, effect sizes, degrees of freedom and $P$ value noted Give $P$ values as exact values whenever suitable.

Х $\square$ For Bayesian analysis, information on the choice of priors and Markov chain Monte Carlo settings

Х $\square$ For hierarchical and complex designs, identification of the appropriate level for tests and full reporting of outcomes

\ $\square$ Estimates of effect sizes (e.g. Cohen's $d$, Pearson's $r$ ), indicating how they were calculated

\section{Our web collection on statistics for biologists contains articles on many of the points above.}

\section{Software and code}

Policy information about availability of computer code

Data collection Aortae were imaged en face using either an LSM880 confocal microscope (ZEISS) or an A1R HD25 confocal microscope (Nikon). Whole Aortae were imaged using an ECHO-Revolve (RVL-100-G)

Flow cytometry was carried out on the Becton Dickinson (BD) Fortessa/ BD FACSDiva v8.0.

Aortae were imaged on a scanning electron microscope JEOL NeoScope.

scRNAseq libraries were generated using the Chromium Single Cell 3' Library \& Gel Bead Kit v2 (10X Genomics). 

image (ZEN 2.0 Black software, ZEISS or NIS-Elements, Nikon).

Imaris software (Imarise 9.5.1, 8.3.1, Bitplane) was used to visualize aorta images in 3D

The digital expression matrix was generated by demultiplexing, barcode processing, and gene unique molecular index counting using the

Cellranger count pipeline (version 2.1.2, 10X Genomics)

The R package Seurat (version 2.3.4) was used to analyze the digital expression matrix.

Flow cytometry analysis was done using FlowJo (9.9.6)

Cell size quantifications were measured using NIS-Elements (Nikon).

Statistical analyses were performed with Excel (2016) or GraphPad Prism (8.02)

Metascape, a gene annotation and analysis resource web tool, was used for gene ontology comparisons

Metascape [http://metascape.org]

BD FACSDiva (8.0.1)

Adobe Illustrator (25.4.1])

Adobe photoshop (22.5.0)

For manuscripts utilizing custom algorithms or software that are central to the research but not yet described in published literature, software must be made available to editors and reviewers. We strongly encourage code deposition in a community repository (e.g. GitHub). See the Nature Portfolio guidelines for submitting code \& software for further information.

\title{
Data
}

Policy information about availability of data

All manuscripts must include a data availability statement. This statement should provide the following information, where applicable:

- Accession codes, unique identifiers, or web links for publicly available datasets

- A description of any restrictions on data availability

- For clinical datasets or third party data, please ensure that the statement adheres to our policy

The scRNA-seq data were deposited in the GEO database, accession number GSE161787

\section{Field-specific reporting}

Please select the one below that is the best fit for your research. If you are not sure, read the appropriate sections before making your selection.

\author{
$\bigotimes$ Life sciences $\quad \square$ Behavioural \& social sciences $\quad \square$ Ecological, evolutionary \& environmental sciences
}

For a reference copy of the document with all sections, see nature.com/documents/nr-reporting-summary-flat.pdf

\section{Life sciences study design}

All studies must disclose on these points even when the disclosure is negative.

Sample size Sampling size for animal experiments was determined by power analysis with a type 1 error rate of $5 \%$, and a minimun detectable effect of $20 \%$. For cell surface experiments we used sampling similar to previous published reports. For experiments where the outcome was immunofluorescence, the figures show representative images; however the number of independent times when the experiment was reproduced using biological replicates is provided in the legend. We avoided too high numbers in order to keep the number of experimental animals as low as possible. All cell and animal-based experiments had at least 3 biological replicates

Data exclusions No samples or animals were excluded.

Replication Every panel in the figure legends indicate the number of biological replicates / independent experiments. Experimental findings were successfully replicated by authors.

Randomization Because the large majority of the experiments required a specific genetic signature, randomization per treatment was not possible. However, the collection and evaluation of the data was blind. Mice were studied as soon as they reach adulthood (8wks) or at the desired ages for developmental studies. Studies were performed using littermate controls.

Blinding

\section{Reporting for specific materials, systems and methods}

We require information from authors about some types of materials, experimental systems and methods used in many studies. Here, indicate whether each material, system or method listed is relevant to your study. If you are not sure if a list item applies to your research, read the appropriate section before selecting a response. 


\begin{tabular}{l|l}
\hline n/a & Involved in the study \\
\hline & $\bigotimes$ Antibodies \\
$\square$ & $\bigotimes$ Eukaryotic cell lines \\
$\square$ & $\square$ Animals and other organisms \\
$\square$ & $\square$ Clinical data \\
$\searrow$ & $\square$ Dual use research of concern
\end{tabular}

\title{
Antibodies
}

Antibodies used

VE-cadherin (1:400), Santa Cruz, sc-6458; AB_2077955/VE-cadherin (1:100), R\&D, AF1002; AB_2077789/CD45 (1:200), BD, \#550539; AB_2174426/ERG (1:400), Abcam, ab92513; AB_2630401/CD11c (1:200), BD, \#550283; AB_393578/CXCL16 (1:200), R\&D, AF503; AB_2230043/MMP13 (1:200), Abcam, ab39012; AB_776416/ MMP12 (1:200), Novus, NBP2-67344/ Fibrinogen (1:400), Abcam, ab118533; AB_10900171/phospho-MLC2 (1:200 IF), (1:500 WB), CST,\#367S; AB_330248/CD45.2-biotin (1:200), BioLegend,109804; AB_313441/CD45-biotin (1:200) ,BD \#554876; AB_395569/CD68-a647 (1:200), BioLegend, 137003; AB_2044001/Thrombin (1:200), Novus,NBP1-58268; AB_11023777/Pecam1 (2H8) (1:1000), Laboratory of William Muller, Bogen et al. 1992; AB_2161039/Pecam1 (390) -Dylight 650 (1:100), Laboratory of William Muller (Millipore),CBL1337-I; AB_2283583/ MLC2 (1:500), CST, 3672S; AB_10692513/Par1 (1:800) Thermo PA5-19102; AB_10986472, gamma-tubulin (1:1000), Abcam, ab11321; AB_297926/CD45-APCCy7 (1:200),BD,557659; AB_396774/Ter119-FITC (1:400), BioLegend, 116215; AB_493402/ Ter119-PEcy7 (1:400), eBioscience, 25-5921-82; AB_469661/Csf1r-BV420 (1:400), BioLegend, 135513; AB_2562667/Cx3cr1-APC (1:400), BioLegend, 149008; AB_2564492//Secondary (1:400): Donkey anti-rabbit a488, Fisher, \#A21206; AB_2535792/ Donkey anti-goat a488, Invitrogen, \#A11055; AB_2534102/Donkey anti-sheep a488, Fisher, \#A11015; AB_2534082/ Donkey anti-rabbit a568, Fisher, A10042; AB_2534017/Donkey anti-goat a568, Fisher, 11057; AB_2534104/ Donkey anti-rabbit a647, Invitrogen, \#31573; AB_2536183/Donkey anti-goat a647, Abcam, ab150135; AB_2687955/Donkey anti-rabbit a405,Abcam,ab175649; AB_2715515/ Goat anti-hamster biotin, BioLegend, 405501; AB_315019/Goat anti-hamster a488, Jackson IR,127-545-160; AB_2338997/Goat antihamster a647, Jackson IR, 127-605-160; AB_2339001/Streptavidin(STA)-a647, BioLegend, \#405237/STA-PE,BioLegend \#405203/ anti-rabbit HRP, Fisher, 45-000-682, AB_772206/ anti-goat HRP, Thermo,31433, AB_228390

Validation

Antibodies were validated by the respective vendors for the applications described in this manuscript. Validation of CD11c, Fibrinogen and Pecam1 were done by immunocytochemistry using a KO mouse for each one of those genes side by side with a wildtype (control) mouse. In the absence of knock-out mice, we used antibodies on western blots and verified a single band at the correct molecular weight.

\section{Eukaryotic cell lines}

\section{Policy information about cell lines}

Cell line source(s)

Human aortic endothelial cells (HAEC) cell lines were isolated as described (Navab et al., 1988) from aortic trimmings of donor hearts at the University of California, Los Angeles (UCLA). All HAECs were de-identified and exempt from consideration as human subjects research by institutional regulatory boards at UCLA. Additional HAECs were purchased from Lonza (\#CC-2535)

Authentication

Mycoplasma contamination

Commonly misidentified lines (See ICLAC register)
Authentification was performed by immunofluorescence for endothelial cell markers (Vecadherin, Pecam1), western blotting, and qPCR as well as alignment in response to laminar flow

In this study, mycoplasma levels were routinely tested in the cell lines used and were mycoplasma free.

none

\section{Animals and other organisms}

Policy information about studies involving animals; ARRIVE guidelines recommended for reporting animal research

Laboratory animals

\begin{abstract}
All mouse strains were maintained on a C57BL/6J background, with the exception of CSF1R-merCremer mice, which was maintained on a mix background (FVB:C57BL/6). Mice were genotyped by Transnetxy. Males and females were used in approximately equal numbers for all experiments with the exception of scRNA-seq experiments. For scRNA-seq experiments, only male C57BL/6 mice were used to minimize sex/strain differences at the transcriptional level for the arch (8wk) and aged descending (78wk) A,B data sets. Unless specified, all adult mice used were 8-10wks of age. Mice information: C57BL/6 (IMSR_JAX:000664)/ VE-cadherin Cre-ERT2 Mice (Tg(Cdh5-cre/ERT2)1Rha ;MGI ID 3848982; Soresnse et al. 2009)/flox-stop-flox-tdTomato Mice (IMSR_JAX:007909)/ CSF1RmerCremer Mice (IMSR_JAX:019098)/ CCR2-/- mice (IMSR_JAX:004999) / CX3cr1-creERT2 mice IMSR_JAX:020940 / CSF1R-flox-stopflox-DTR (IMSR_JAX:024046)/ SM22-cre (IMSR_JAX:004746)/ Flt3-cre;R26-flox-Tomato-stop-flox-GFP (Laboratory of Anna E. Beaudin; Boyer et al. 2011)/ Fibrinogen-/- mice (Laboratory of Matthew Flick, Bugge et al. 1996)/ Fibrinogen-AEK mice (Laboratory of Matthew Flick, Prasad et al. 2015)/ CD11c-/- (Laboratory of Matthew Flick)/ R26-Rainbow (Laboratory of Reza Ardehali, Rinkevich et al. 2011). Mouse housing conditions are as follows: Light cycle is $14 / 10$ (lights go on at 6am and off at 8pm). Humidity range is $30-70 \%$. Temperature is 72 plus or minus 2 (range is $70-74 \mathrm{~F}$ ).
\end{abstract}


Field-collected samples

No field-collected samples were used in this study

Ethics oversight

All animal procedures were approved and performed in accordance with the UCLA and Northwestern University Institutional Anima Care and Use Committee protocol number ISO0013945.

Note that full information on the approval of the study protocol must also be provided in the manuscript.

\section{Flow Cytometry}

\section{Plots}

Confirm that:

$\bigotimes$ The axis labels state the marker and fluorochrome used (e.g. CD4-FITC).

\The axis scales are clearly visible. Include numbers along axes only for bottom left plot of group (a 'group' is an analysis of identical markers).

\All plots are contour plots with outliers or pseudocolor plots.

\A numerical value for number of cells or percentage (with statistics) is provided.

\section{Methodology}

Sample preparation

Blood was collected by retro-orbitally bleeding into tubes containing FACS buffer at $4 \mathrm{oC}$. Blood cells were pelleted and treated with $1 \mathrm{X}$ RBC lysis buffer. Additionally, cells were stained on ice with antibodies and then analyzed on a BD Fortessa. Flow cytometry of organs: One-day post-diphtheria toxin injection (MacAIR-depletion), littermate control and Cx3cr1creERT2;CSF1RIsIDTR were euthanized and perfused with $10 \mathrm{~mL}$ of versene to remove blood and then perfused with $10 \mathrm{mLs}$ of DMEM. Lung, liver, kidney, and leg bone (femur and tibia) were carefully dissected and washed in PBS. For bone marrow harvest, muscle was removed and bone was cleaned. Bone was then crushed in FACs Buffer using a motor pestle to release bone marrow cells. Bone marrow cells were then pelleted and treated with $1 X$ RBC lysis buffer. For generation of single-cell suspension from lung, liver, and kidney, tissues were digested using Militenyi's lung dissociation kit (\#130-095-927), liver dissociation kit (\#130-106-807), and multidissociation kit \#2 (\#130-110-203), following vendor's protocol. Cells were pelleted and treated with $1 \mathrm{X}$ RBC lysis buffer. Additionally, bone marrow, lung, liver, and kidney cells were stained on ice with CD45-APC-Cy7 (BD, \#557659), CX3CR1-APC (BioLegend, \#149008), CSF1R-BV421 (BioLegend, \#135513), and Ter119-PECy7 (eBioscience, \#25-5921-82) then analyzed on a BD Fortessa.

Instrument

Becton Dickinson (BD) Fortessa

Software

BD FACSDiva v8.0, FlowJo(9.9.6)

Cell population abundance

Flow cytometry was used only for analysis. No FACS sorting.

Gating strategy

Cells were gated on singlets (standard gating strategy), size selection (forward/side scatter), and antibody staining.

$\bigotimes$ Tick this box to confirm that a figure exemplifying the gating strategy is provided in the Supplementary Information. 